\title{
NAVIER-STOKES EQUATIONS IN THIN 3D DOMAINS WITH NAVIER BOUNDARY CONDITIONS
}

\author{
DRAGOŞ IFTIMIE, GENEVIÈVE RAUGEL, AND GEORGE R. SELL
}

\begin{abstract}
We consider the Navier-Stokes equations on a thin domain of the form $\Omega_{\varepsilon}=$ $\left\{x \in \mathbb{R}^{3} ; x_{1}, x_{2} \in(0,1), 0<x_{3}<\varepsilon g\left(x_{1}, x_{2}\right)\right\}$ supplemented with the following mixed boundary conditions: periodic boundary conditions on the lateral boundary and Navier boundary conditions on the top and the bottom. Under the assumption that $\left\|u_{0}\right\|_{H^{1}\left(\Omega_{\varepsilon}\right)} \leq C \varepsilon^{-\frac{1}{2}},\left\|M u_{0}^{i}\right\|_{L^{2}\left(\Omega_{\varepsilon}\right)} \leq C$ for $i \in\{1,2\}$ and similar assumptions on the forcing term, we show global existence of strong solutions; here $u_{0}^{i}$ denotes the $i$-th component of the initial data $u_{0}$ and $M$ is the average in the vertical direction, that is, $M u_{0}^{i}\left(x_{1}, x_{2}\right)=\frac{1}{\varepsilon g} \int_{0}^{\varepsilon g} u_{0}^{i}\left(x_{1}, x_{2}, x_{3}\right) d x_{3}$. Moreover, if the initial data, respectively the forcing term, converge to a bidimensional vector field, respectively forcing term, as $\varepsilon \rightarrow 0$, we prove convergence to a solution of a limiting system which is a Navier-Stokes-like equation where the function $g$ plays an important role. Finally, we compare the attractor of the Navier-Stokes equations with the one of the limiting equation.
\end{abstract}

KEY WORDS: Navier-Stokes equations, thin domain, global existence, global regularity, global attractor.

AMS subJeCt Classification: Primary 35Q30, 76D05, 46E35; Secondary 35B65, 35K55.

\section{INTRODUCTION}

Thin domains are encountered in the study of many problems in science, for example in solid mechanics (thin rods, plates or shells), in fluid dynamics (lubrication, meteorology, geophysical problems, ocean dynamics) and in physiology (blood circulation). In ocean or great lakes dynamics, one is dealing with large scale fluid problems, where the fluid regions are thin compared to the horizontal length scales.

Most of the above problems are described by a partial differential equation (PDE) on a thin domain. A natural temptation is thus to exploit the thinness in one or several directions of the domain in order to get a better understanding of the properties of the solutions of this PDE. For example, let us suppose that we are given a partial differential evolutionary equation $\left(P_{\varepsilon}\right)$ with homogeneous Neumann type boundary conditions on an $(n+k)$-dimensional thin product domain $\Omega_{\varepsilon}=Q_{n} \times(0, \varepsilon)^{k}$, where $Q_{n}$ is a smooth $n$ dimensional domain. In such a situation, it is natural to decompose the solution $u(t)$ of the problem $\left(P_{\varepsilon}\right)$ into a sum of two functions $v(t)+w(t)$, where $v(t)$ is the average of $u(t)$ in the thin directions and where the average of $w(t)$ in the thin directions vanishes. Since $v(t)$ depends only on $n$ spatial variables, one may use better Sobolev, Agmon, etc. inequalities in estimating expressions involving $v(t)$. On the other hand, $w(t)$ satisfies good Poincaré type estimates, which leads to enhanced stability in $\Omega_{\varepsilon}$. Considering the 
equations satisfied by $v(t)$ and $w(t)$ and using the good properties of $v(t)$ and $w(t)$, one obtains a better information on the solution $u(t)$.

Another way to proceed is to consider the restriction $\left(P_{0}\right)$ of the evolutionary equation to the lower dimensional domain $Q_{n}$ and to compare the dynamics of $\left(P_{\varepsilon}\right)$ with the dynamics of $\left(P_{0}\right)$. In the case of dissipative evolutionary equations having a global attractor $\mathcal{A}_{\varepsilon}$ one may also compare the set $\mathcal{A}_{\varepsilon}$ with the global attractor $\mathcal{A}_{0}$ of the limit equation. The equation $P_{0}$ on the lower dimensional domain $Q_{n}$ is often referred to as limiting equation or reduced equation. Such questions have been studied by Hale and Raugel $[12,13]$ for reactiondiffusion and damped wave equations for more general thin domains. Both strategies are still valid for more general thin domains $\Omega_{\varepsilon}$, that are no longer product domains (see [32] for references and more details)

As we will see below, such strategies also apply to the Navier-Stokes equations on thin three-dimensional domains. Let us recall that global existence of weak solutions of the Navier-Stokes equations is known to hold in every space dimension. Uniqueness of weak solutions (and global existence of strong solutions) is known in dimension two ([18]). In dimension three, global existence of strong solutions is known only under additional smallness assumptions on the initial data and the forcing term. Thus a natural question arises, namely can we use the thinness of the three-dimensional domain in order to improve the global existence results of strong solutions?

The study of the global existence of strong solutions of the Navier-Stokes equations in thin three-dimensional domains began in the early 1990s with the papers [33] and [34], by Raugel and Sell. Inspired by methods developed in [13] and [12], they proved global existence of strong solutions for large initial data $u_{0}$ and forcing term $f$, in the case of thin three-dimensional "product domains" $\Omega_{\varepsilon}=Q_{2} \times(0, \varepsilon)$, when the boundary conditions are either purely periodic (PP) (in which case $Q_{2}$ is the two-dimensional torus $\mathbb{T}^{2}$ ) or periodicDirichlet $(\mathrm{PD})$, that is, periodic conditions in the vertical thin direction and homogeneous Dirichlet boundary conditions on the lateral boundary $\partial Q_{2} \times(0, \varepsilon)$. An essential ingredient in their proof was the decomposition of every vector $u$ into the sum of a vector $v=M u$, independent of the vertical variable and a vector $w=(I-M) u$ with vertical vanishing mean, which allows to use 2D Sobolev estimates on the nonlinear terms involving the vector $v$ and the fact that the lower Sobolev norms of $w$ are small compared to the higher ones (see Lemma 3.10). In the case of a thin product domain, using the same methods as Raugel and Sell and also anisotropic Agmon inequalities, Temam and Ziane [44] generalized the results of [33] and [34] to other boundary conditions, namely (FF), (FP) and (FD) boundary conditions, where $(\mathrm{F})$ means free boundary conditions. It should be noted that the free boundary conditions coincide with the Navier boundary conditions (1.3) if and only if the boundary is a part of a hyperplane, see [2] for more details. Later, in the periodic case, global existence of strong solutions has been proved for initial data that are larger than in [34] by Moise, Temam and Ziane [24] (see also Montgomery-Smith [25]). Using the anisotropic Sobolev spaces $H^{s, s^{\prime}}\left(\Omega_{\varepsilon}\right)$ and the Littlewood-Paley theory, Iftimie [14] showed global existence and uniqueness of solutions for initial data $u_{0}$ such that $M u_{0}$ is only square integrable and $(I-M) u_{0}$ belongs to an anisotropic Sobolev space of total regularity $H^{\frac{1}{2}}$; moreover, the part $(I-M) u_{0}$ is larger than in the above papers. Finally, in the cases of $(\mathrm{PP}),(\mathrm{FP}),(\mathrm{FF})$, all the previous existence and uniqueness results have been improved by 
Iftimie and Raugel in [15] in two directions, by requiring less regularity on the initial data and by allowing a larger size of the initial data and forcing term. These improvements were due, on one hand, to sharp estimates of the nonlinear term appearing in the NavierStokes equations and, on the other hand, to the use of commutator properties. In all the above mentioned papers, the decomposition of the solution $u$ into the sum $v+w$ played an important role. Another main ingredient in the proofs of these papers is the conservation of the enstrophy for the underlying 2D problem in the cases of $(\mathrm{PP}),(\mathrm{FF}),(\mathrm{PF}),(\mathrm{FP})$ boundary conditions; this conservation of enstrophy allows much larger initial data and forcing terms than in the case of (PD) or (FD) boundary conditions.

Finally let us mention that the analysis in the case of homogeneous Dirichlet boundary conditions (DD), (DP), (DF) in a thin domain is much simpler since the size of the first eigenvalue is of order $\varepsilon^{-2}$ and thus there is no need of the decomposition of $u$ into $v+w$ (see [1], [44]). More details concerning the specific contributions of most of the papers cited above can be found in [15] (pages 282 to 294).

The following is a brief summary of the types of results which can be found in the above mentioned sources:

- It is shown that there exist "large" open neighbourhoods $N_{\varepsilon}^{1}$ and $N_{\varepsilon}^{2}$ of the origin in the function space $V_{\varepsilon}$ of solenoidal vector fields in $H^{1}\left(\Omega_{\varepsilon}\right)^{3}$ and in the function space of forcing terms in $L^{\infty}\left(0, \infty ; L^{2}\left(\Omega_{\varepsilon}\right)^{3}\right)$ respectively, with the property that if the data $\left(u_{0}, f\right)$ for the Navier-Stokes equations lie in $N_{\varepsilon}=N_{\varepsilon}^{1} \times N_{\varepsilon}^{2}$, then there exists a unique globally defined strong solution to the Navier-Stokes equations. In the cases of (PP), (FF), (PF), (FP) boundary conditions, these neighbourhoods $N_{\varepsilon}^{1}$ and $N_{\varepsilon}^{2}$ are much larger, due to the conservation of enstrophy for the underlying 2D Navier-Stokes problem.

- When $f=f(x)$ is time-independent, then there exists a compact (local) attractor $\mathcal{A}_{\varepsilon}$ in $V_{\varepsilon}$ of all the strong solutions of the Navier-Stokes equations in $V_{\varepsilon}$ and $B\left(\mathcal{A}_{\varepsilon}\right)$, the basin of attraction of $\mathcal{A}_{\varepsilon}$, contains $N_{\varepsilon}^{1}$. Furthermore, the following properties hold:

(a) as usual, the solutions of the Navier-Stokes equations in the attractor $\mathcal{A}_{\varepsilon}$ are globally defined for all $t \in \mathbb{R}$;

(b) in addition, the (local) attractor $\mathcal{A}_{\varepsilon}$ is the global attractor of all the weak Leray-Hopf solutions of the Navier-Stokes equations.

Additional features concerning the attractor, in the case where the forcing function $f=f(x, t)$ does depend on time, can be found in [35].

It is noteworthy that in the above cited papers about global existence of regular solutions on thin 3D domains, there is a common feature, namely, the simple geometry of the underlying "physical" space. Roughly speaking, it is assumed that $\Omega_{\varepsilon}=Q_{2} \times(0, \varepsilon)$ is the product of a suitable 2-dimensional bounded domain $Q_{2}$ in $\mathbb{R}^{2}$ and an interval $(0, \varepsilon)$, where $\varepsilon$ is positive, but small. However, in the physical problems to which we alluded above, like in oceanic flows on the Earth, the geometry is more complex. A first step to a more general geometry has been made by Temam and Ziane [45], who studied the Navier-Stokes equations with free boundary conditions in a thin spherical shell. Another generalisation of simple product domains was made by Chueshov, Raugel, and Rekalo, [3], who considered a thin product domain with interface conditions. 
In shallow water problems or in geophysical problems involving flows in great lakes or in oceans (see for instance [19]) where the depth of the water is small compared to the width, the bottom topography (described by the function $g$ introduced below) is not negligible and plays an important role. For this reason, in the present paper, we consider the NavierStokes equations in a thin domain described by such a function $g$ (see (1.1)).

Another novelty of this article is the consideration of the Navier boundary conditions, see (1.3). Unlike the earlier studies, which were based on periodic or free boundary conditions, we now study the Navier-Stokes equations on a domain with a "non-flat" boundary and with the Navier boundary conditions.

The Navier boundary conditions appear already in the original paper of Navier [26], see also the remarks of Serrin [40] (In the literature, the Navier boundary conditions are sometimes referred to as "stress-free" or "slip" boundary conditions). The stationary Navier-Stokes equations with mixed Navier and Dirichlet boundary conditions have been studied in [41]. These boundary conditions arise in the study of climate modeling and oceanic flows, see [22], [30] for example. In [22], Lions, Temam and Wang introduce the Navier conditions in terms of an interface condition (see [3] for the study of such an interface condition in the case of a thin product domain). Also a version of the Navier conditions with a friction coefficient arises in $[16,17]$, and is related to the homogenization of the Navier-Stokes equations over a rough boundary. It is also known that the Navier boundary conditions do not lead to boundary layers in the vanishing viscosity limit, see [5, 23]. A numerical study of the Navier-Stokes equations with Navier boundary conditions appears in [46]. We finally note that this type of boundary conditions have applications in turbulence modeling, see [29].

Let $g: \mathbb{T}^{2}=[0,1]^{2} \rightarrow \mathbb{R}_{+}$be a periodic function of class $C^{3}$ such that there exist two positive constants $C_{g}>c_{g}>0$ with $c_{g} \leq g \leq C_{g}$. We consider the domain

$$
\Omega_{\varepsilon}=\left\{\left(x_{h}, x_{3}\right): x_{h}=\left(x_{1}, x_{2}\right) \in \mathbb{T}^{2}, 0 \leq x_{3} \leq \varepsilon g\left(x_{h}\right)\right\}, \quad \text { where } 0<\varepsilon \leq 1 .
$$

All functions are assumed to be periodic in $x_{1}$ and $x_{2}$. As a consequence, the boundary of $\Omega_{\varepsilon}$ can be decomposed as follows:

$$
\partial \Omega_{\varepsilon}=\Gamma_{\varepsilon} \cup \Gamma_{0}
$$

where

$$
\Gamma_{\varepsilon}=\left\{\left(x_{h}, \varepsilon g\left(x_{h}\right)\right): x_{h} \in \mathbb{T}^{2}\right\} \quad \text { and } \quad \Gamma_{0}=\left\{\left(x_{h}, 0\right): x_{h} \in \mathbb{T}^{2}\right\} .
$$

We consider the Navier-Stokes equations

$$
\partial_{t} u-\nu \triangle u+u \cdot \nabla u=f-\nabla p, \quad \operatorname{div} u=0,
$$

with initial condition $\left.u\right|_{t=0}=u_{0}=u(0)$ and supplemented with the following mixed periodic-Navier boundary conditions:

$$
\begin{gathered}
u \text { periodic in } x_{1} \text { and } x_{2} \\
\left.u \cdot N\right|_{\Gamma_{0} \cup \Gamma_{\varepsilon}}=0,\left.\quad[D(u) N]_{\tan }\right|_{\Gamma_{0} \cup \Gamma_{\varepsilon}}=0,
\end{gathered}
$$

where $D(u)$ is the deformation tensor and is defined by $[D(u)]_{i, j}=\left(\partial_{i} u_{j}+\partial_{j} u_{i}\right) / 2$, where $N$ is the exterior normal and $[D(u) N]_{\tan }$ is the tangential component of the vector field 
$D(u) N$. In other words, we ask the velocity to be tangent to the boundary and the vector $D(u) N$ to be normal to the boundary.

Before stating our Main Theorem, we introduce some notation. We need to impose some additional conditions on the forcing term and on the initial data, which will be made explicit in Section 2 (see conditions $(\mathrm{H})$ and $\left(\mathrm{H}_{f}\right)$ ). In the case of a constant function $g$, these conditions reduce to the vanishing of the total mean value of the horizontal components of the initial data and forcing term. Thus we introduce the spaces $H_{\varepsilon}$ and $V_{\varepsilon}$, where

$$
\begin{array}{r}
H_{\varepsilon}=\left\{U \in L^{2}\left(\Omega_{\varepsilon}\right)^{3} \mid U \text { is periodic in } x_{1} \text { and } x_{2}, U \cdot N=0 \text { on } \Gamma_{\varepsilon} \cup \Gamma_{0},\right. \\
\operatorname{div} U=0, U \text { satisfies the condition(H) }\},
\end{array}
$$

and

$$
V_{\varepsilon}=H_{\varepsilon} \cap H^{1}\left(\Omega_{\varepsilon}\right)^{3} .
$$

Let $\mathbb{P}_{\varepsilon}$ denote the classical Helmholtz-Leray (orthogonal) projection of $L^{2}\left(\Omega_{\varepsilon}\right)^{3}$ onto $H_{\varepsilon}$.

During the course of our analysis, we will encounter various norms, such as $\|\cdot\|_{L^{2}}$, $\|\cdot\|_{H^{1}},\|\cdot\|_{H^{2}},\|\cdot\|_{L^{q}}$, etc. In such cases, the norms are to be calculated by integration over the three-dimensional domain $\Omega_{\varepsilon}$. Thus the subscripts should read as: $L^{2}=L^{2}\left(\Omega_{\varepsilon}\right)^{3}$, $H^{1}=H^{1}\left(\Omega_{\varepsilon}\right)^{3}$, etc. In other cases, where the domain of integration is not $\Omega_{\varepsilon}$, we will use the expanded notation for the subscripts. For example, in the case of functions that are defined on the torus $\mathbb{T}^{2}$ and that do not depend on the $x_{3}$-variable, we will express the subscripts for the norms in the form $L^{2}\left(\mathbb{T}^{2}\right), H^{1}\left(\mathbb{T}^{2}\right)$, etc. if the integration is made over the domain $\mathbb{T}^{2}$ instead of $\Omega_{\varepsilon}$. Lastly, for functions $f=f(t, x)$ in $L^{\infty}\left((0, \infty) ; L^{2}\left(\Omega_{\varepsilon}\right)^{3}\right)$, we define the norm $\|f\|_{\infty}$ by

$$
\|f\|_{\infty}=\sup _{t>0}\|f(t, \cdot)\|_{L^{2}\left(\Omega_{\varepsilon}\right)},
$$

where - as usual - one uses the essential supremum.

In the theorem stated next, we note that $v_{h}=M_{h} u=\left(M u_{1}, M u_{2}, 0\right)$ is the horizontal component of the vector field $M u \equiv\left(M u_{1}, M u_{2}, M u_{3}\right)$, where

$$
M \phi=M \phi\left(x_{1}, x_{2}\right)=\frac{1}{\varepsilon g} \int_{0}^{\varepsilon g} \phi\left(x_{1}, x_{2}, x_{3}\right) d x_{3},
$$

for any $L^{1}$-scalar-valued function $\phi$ defined on $\Omega_{\varepsilon}$.

Arguing as in the case of classical Dirichlet boundary conditions, one shows (see Section 2.2 below) that, for any forcing term $f \in L^{\infty}\left((0,+\infty) ; L^{2}\left(\Omega_{\varepsilon}\right)^{3}\right)$ satisfying the compatibility condition $\left(\mathrm{H}_{f}\right)$, and for any initial datum $u_{0} \in V_{\varepsilon}$, there exist a positive time $T^{*}=$ $T^{*}\left(\Omega_{\varepsilon}, \nu, u_{0}, f\right)$ and a unique strong solution $u \in C^{0}\left(\left[0, T^{*}\right), V_{\varepsilon}\right)$ of (1.2). Furthermore, using a classical small data argument, one shows that, if

$$
\left\|u_{0}\right\|_{H^{1}}+\|f\|_{\infty} \leq C \varepsilon^{1 / 2}
$$

where $C$ is independent of $\varepsilon$, then the solution of (1.2) is global in time, that is, $T^{*}=+\infty$. We show in Theorem 1.1 that, exploiting the fact that $\Omega_{\varepsilon}$ is a thin domain, we can replace the small data condition on $\left(u_{0}, f\right)$ by large data conditions.

Theorem 1.1. There exist positive constants $\varepsilon_{0}, \kappa_{0}, \kappa_{1}, K_{0}, K_{1}$ and $R^{*}$ such that, for any $\varepsilon$ with $0<\varepsilon \leq \varepsilon_{0}$, for any forcing term $f \in L^{\infty}\left((0,+\infty) ; L^{2}\left(\Omega_{\varepsilon}\right)^{3}\right)$ satisfying the 
compatibility condition $\left(\mathrm{H}_{f}\right)$, and for any initial datum $u_{0} \in V_{\varepsilon}$, that satisfy

$$
\begin{gathered}
\left\|u_{0}\right\|_{H^{1}\left(\Omega_{\varepsilon}\right)} \leq \kappa_{1} \varepsilon^{-1 / 2}, \quad\left\|M_{h} u_{0}\right\|_{L^{2}\left(\Omega_{\varepsilon}\right)} \leq \kappa_{0} \\
\|f\|_{\infty} \leq K_{1} \varepsilon^{-1 / 2}, \quad\left\|M_{h} f\right\|_{\infty} \leq K_{0},
\end{gathered}
$$

the Navier-Stokes equations (1.2)-(1.3) have a unique global strong solution u(t) with

$$
u(t) \in C^{0}\left([0, \infty), V_{\varepsilon}\right) \cap L^{\infty}\left((0, \infty), V_{\varepsilon}\right) \cap L_{l o c}^{2}\left((0, \infty), H^{2}\left(\Omega_{\varepsilon}\right)\right) \cap H_{l o c}^{1}\left((0, \infty), H_{\varepsilon}\right),
$$

and

$$
\|u(t)\|_{H^{1}\left(\Omega_{\varepsilon}\right)} \leq R^{*} \varepsilon^{-1 / 2}, \quad \text { for all } t \geq 0
$$

Remark 1.1. Since $f-\mathbb{P}_{\varepsilon} f$ is a gradient, the conclusions of Theorem 1.1 are still valid under the hypothesis, where $f$ and $M_{h} f$ are replaced by $\mathbb{P}_{\varepsilon} f$ and $M_{h} \mathbb{P}_{\varepsilon} f$ in the conditions (1.5).

Before making comments about the size of the data, we want to emphasize that the arguments in the proof of Theorem 1.1 differ from earlier proofs for the Navier-Stokes equations on thin 3D-domains (see [33], [44], and [15]). The use of vertical averaging operators, like $M$ in (1.4) and $M_{h}$ or $M_{1}$ in (3.1) and (3.2), is a feature which is similar to related usage appearing in the papers cited above. However, there are new complexities which arise due to the non-flat boundary $\Gamma_{\varepsilon}$ (see (1.1)) and to the Navier boundary conditions (1.3) used herein. Indeed, in the earlier papers, like in [33], [44], [45], and [15], the vertical average operator commutes with the Stokes operator $A_{\varepsilon}=-\mathbb{P}_{\varepsilon} \Delta$, which is no longer true here. More precisely, let $u \in H^{2}\left(\Omega_{\varepsilon}\right)^{3}$ satisfy the Navier boundary conditions and assume that $\operatorname{div} u=0$ in $\Omega_{\varepsilon}$. Then $u \in D\left(A_{\varepsilon}\right)$. However, none of the vectors fields, $M_{h} u, M_{1} u$, $\left(I-M_{h}\right) u,\left(I-M_{1}\right) u$, described above are in $D\left(A_{\varepsilon}\right)$ when $\nabla g \not \equiv 0$. The problem is that if $U$ is one of these four vectors, then $(D(U) N)_{\tan } \neq 0$ on $\Gamma_{\varepsilon}$, when $\nabla g \not \equiv 0$.

In these earlier papers, the fact that the mean value $M_{h}$ commutes with the Stokes operator had several advantages. One could decompose the $u$-equation (1.2) into a system of $(v, w)$-equations in $D\left(A_{\varepsilon}\right)$, which offered several important advantages:

- the eigenvalues of the Stokes operator $A_{\varepsilon}$ split into separate eigenvalues for $M A_{\varepsilon}$ and $(I-M) A_{\varepsilon}$;

- the eigenvalues for $(I-M) A_{\varepsilon}$ are positive and very large;

- one can show that the solutions of the linear $w$-equation are exponentially stable with very large decay rate.

Unfortunately, none of these advantages are available in this study. Nevertheless, as we shall see, there is no reason to decompose the $u$-equation (1.2) into a system of $(v, w)$ equations. Another property is missing in the problem that we are studying here. While the "conservation of enstrophy property" holds in the case of the boundary conditions (PP), $(\mathrm{PF}),(\mathrm{FP})$, and $(\mathrm{FF})$, when $\Omega_{\varepsilon}$ is a product domain and in the case of free boundary conditions when $\Omega_{\varepsilon}$ is a spherical shell, it does not appear to hold for our problem.

Next, several remarks have to be made concerning the conditions (1.5) on the size of the initial data. 
Remark 1.2. It is important to note the scaling in (1.5). If $\phi \in L^{2}\left(\mathbb{T}^{2}\right)$, then $\phi \in L^{2}\left(\Omega_{\varepsilon}\right)$, and one has

$$
c_{g}\|\phi\|_{L^{2}\left(\mathbb{T}^{2}\right)}^{2} \leq \varepsilon^{-1}\|\phi\|_{L^{2}\left(\Omega_{\varepsilon}\right)}^{2} \leq C_{g}\|\phi\|_{L^{2}\left(\mathbb{T}^{2}\right)}^{2},
$$

with a similar inequality valid when $\phi$ is a vector field in the space $L^{2}\left(\mathbb{T}^{2}\right)^{2}$. In the case where $\phi=M_{h} u_{0}$ and (1.5) hold, $\left\|M_{h} u_{0}\right\|_{L^{2}\left(\mathbb{T}^{2}\right)^{2}}$ can be very large of order $\varepsilon^{-1 / 2}$, for small $\varepsilon$. Furthermore, if $M_{h} u_{0}$ belongs to $L^{\infty}\left(\Omega_{\varepsilon}\right)^{2}$, the conditions (1.5) allow the norm $\left\|M_{h} u_{0}\right\|_{L^{\infty}\left(\mathbb{T}^{2}\right)^{3}}$ to be of order $\varepsilon^{-1 / 2}$.

Remark 1.3. Our conditions (1.5) should be compared to those imposed on the initial data and forcing term in the case of free boundary conditions when $\Omega_{\varepsilon}$ is either a thin product domain or a spherical shell. In [15] (see Remark 1.4), Iftimie and Raugel proved the following existence result, in the case of (FP) boundary conditions when $\Omega_{\varepsilon}=\mathbb{T}^{2} \times(0, \varepsilon)$. There, it was remarked that there exist positive constants $k_{1} k_{2}, k_{3}$, and $\epsilon_{0}$ such that for any $\varepsilon$ with $0<\varepsilon \leq \varepsilon_{0}$, for any forcing term $f \in L^{\infty}\left((0,+\infty) ; L^{2}\left(\Omega_{\varepsilon}\right)^{3}\right)$ satisfying the compatibility condition $\left(\mathrm{H}_{f}\right)$ (for $g \equiv 1$ ), and for any initial datum $u_{0} \in V_{\varepsilon}$, that satisfy

$$
\begin{aligned}
& \left\|u_{0}\right\|_{H^{1}} \leq k_{1} \varepsilon^{-1 / 2}, \\
& \left\|M_{h} f\right\|_{\infty} \leq k_{2} \varepsilon^{-1 / 2}, \quad\left\|\left(I-M_{h}\right) f\right\|_{\infty} \leq k_{3} \varepsilon^{-1},
\end{aligned}
$$

the Navier-Stokes equations with (FP) boundary conditions have a unique globally defined strong solution $u(t) \in C^{0}\left([0, \infty), V_{\varepsilon}\right)$.

In [45] (see Theorem A), Temam and Ziane showed that, given a function $R_{0}(\varepsilon)>0$ and a positive number $q<\frac{1}{2}$ such that $\varepsilon^{q} R_{0}(\varepsilon)$ tends to zero when $\varepsilon$ goes to 0 , there exists $\varepsilon_{0}>0$ such that, for any $\varepsilon$ with $0<\varepsilon \leq \varepsilon_{0}$, for any forcing term $f \in L^{\infty}\left((0,+\infty) ; L^{2}\left(\Omega_{\varepsilon}\right)^{3}\right)$ and for any initial datum $u_{0} \in V_{\varepsilon}$, that satisfy

$$
\left\|u_{0}\right\|_{H^{1}}^{2}+\|f\|_{\infty}^{2} \leq R_{0}^{2}(\varepsilon)
$$

the Navier-Stokes equations with free boundary conditions on the spherical shell have a unique globally defined strong solution $u(t) \in C^{0}\left([0, \infty), V_{\varepsilon}\right)$, where $V_{\varepsilon}$ is the corresponding subspace of $H^{1}\left(\Omega_{\varepsilon}\right)^{3}$ of solenoidal vector fields. Let us point out that the global existence of strong solutions in the case of a thin spherical shell with free boundary conditions should still hold if the conditions (1.7) are replaced by the less restrictive conditions (1.6).

Comparing the hypotheses (1.5) to the conditions (1.6) or (1.7), one sees that we need an additional condition on the $L^{2}\left(\Omega_{\varepsilon}\right)$-norm of $M_{h} u_{0}$. This additional condition is due to the fact that, unlike in the simpler cases above, there is apparently no conservation of the enstrophy for the corresponding 2D Navier-Stokes equations (6.5) (see also the Proposition $4.2)$.

In the case of a thin product domain $\Omega_{\varepsilon}=Q_{2} \times(0, \varepsilon)$, the projection $M_{h}$ commutes with the Stokes operator $A_{\varepsilon}$ and the eigenvalues for $\left(I-M_{h}\right) A_{\varepsilon}$ are large of order $\varepsilon^{-2}$. Considering the equation satisfied by $\left(I-M_{h}\right) A_{\varepsilon} u=A_{\varepsilon}\left(I-M_{h}\right) u$ and using this property of eigenvalues allow to relax the condition $\left\|\left(I-M_{h}\right) f\right\|_{L^{2}} \leq C \varepsilon^{-1 / 2}$ into $\left\|\left(I-M_{h}\right) f\right\|_{L^{2}} \leq$ $C \varepsilon^{-1}$. Unfortunately, as we already explained, in the case where the boundary $\Gamma_{\varepsilon}$ is not flat (that is, in the case where $\nabla g \neq 0$ ), there is no appropriate vertical mean operator $M^{*}$ commuting with the Stokes operator $A_{\varepsilon}$ and the eigenvalues of $\left(I-M^{*}\right) A_{\varepsilon}$ are not necessarily large of order $\varepsilon^{-2}$. 
Remark 1.4. Theorem 1.1 can be made more precise with the following remarks about the asymptotic behaviour in time of the globally defined strong solutions of (1.2). Let us fix a time-independent forcing term $f$ satisfying the hypotheses of Theorem 1.1 and let us introduce the local semigroup $S_{\varepsilon}(t)$ defined by $S(t) u_{0}=u(t)$, where $u(t)$ is the local strong solution of the Navier-Stokes equations (1.2) with initial data $u_{0}$. Theorem 1.1 implies that $S_{\varepsilon}(t)$ is a semi flow on the bounded set $\mathcal{B}_{\varepsilon}=\overline{\bigcup_{t \geq 0} S_{\varepsilon}(t) \mathcal{B}_{0, \varepsilon}} V_{\varepsilon}$, where

$$
\mathcal{B}_{0, \varepsilon}=\left\{u_{0} \in V_{\varepsilon} \mid\left\|u_{0}\right\|_{H^{1}} \leq \kappa_{1} \varepsilon^{-1 / 2},\left\|M_{h} u_{0}\right\|_{L^{2}} \leq \kappa_{0}\right\} .
$$

In Section 7, we will show that the dynamical system $S_{\varepsilon}(t)$ restricted to the bounded set $\mathcal{B}_{\varepsilon}$ admits a compact attractor $\mathcal{A}_{\varepsilon}$ in $V_{\varepsilon}$, and that $\mathcal{A}_{\varepsilon}$ is the global attractor of all weak Leray-Hopf solutions (see also [8], [38] and [3], [33] in the case of thin product domains). We will also compare this attractor $\mathcal{A}_{\varepsilon}$ with the global attractor $\mathcal{A}_{0}$ of the limit equation (1.8).

In the case of a non-flat thin domains, another new phenomenon arises, namely the limiting equation, as $\varepsilon$ goes to zero, is much more interesting. For example, in the case of a thin product domain, the limit of the 3D Laplace operator $\Delta=\sum_{i=1}^{3} \partial_{i}^{2}$ (with homogeneous Neumann boundary conditions) is simply the 2D Laplace operator $\Delta_{2}=\sum_{i=1}^{2} \partial_{i}^{2}$. In the case of the above defined non-flat thin domain $\Omega_{\varepsilon}$, it has been proved by Hale and Raugel in [13] and [12] that the limit is the following Laplace-Beltrami operator $L_{g}$ associated to the metric induced by the function $g$, where

$$
L_{g} \varphi=\frac{1}{g} \sum_{i=1}^{2} \partial_{i}\left(g \partial_{i} \varphi\right) .
$$

For the expression of the limit of the Laplace operator in more general thin domains, like thin domains around a manifolds etc., we refer to the following papers [11], [31], [37], [32], [47], for example.

The limiting equations in the case of the Stokes or Navier-Stokes problems are even more interesting, since the function $g$ also appears in the limit of the divergence operator and that, in addition to the above operator $L_{g}$, there are other terms in the evolutionary equation. Before giving the limit equation, we need to introduce some additional notation. If $v$ is any smooth enough two-dimensional vector field defined on $\mathbb{T}^{2}$, we denote its divergence by $\operatorname{div}_{\mathrm{h}} v=\partial_{1} v_{1}+\partial_{2} v_{2}$. Likewise, for any function $\varphi$ defined on $\mathbb{T}^{2}$, we denote by $\nabla_{h} \varphi$ the two-dimensional vector $\left(\partial_{1} \varphi, \partial_{2} \varphi\right)$.

Assume next that the forcing term $f=f_{\varepsilon}$ depends on $\varepsilon$ and that $M_{h} f_{\varepsilon}$ converges in the space $\left.L^{\infty}\left((0, \infty), L^{2}\left(\mathbb{T}^{2}\right)^{3}\right)\right)$ to a force $\left(f_{0}, 0\right)$ where $f_{0}$ belongs to $\left.L^{\infty}\left((0, \infty), L^{2}\left(\mathbb{T}^{2}\right)^{2}\right)\right)$. Then the limit of the 3D Navier-Stokes equations (1.2) is the the following Navier-Stokes type equations

$$
\begin{aligned}
& \partial_{t} v-\frac{\nu}{g} \sum_{i=1}^{2} \partial_{i}\left(g \partial_{i} v\right)+\frac{\nu}{g^{2}} \sum_{i=1}^{2} v_{i} \partial_{i}\left(g \nabla_{h} g\right)+v \cdot \nabla_{h} v=f_{0}-\nabla_{h} p, \\
& \operatorname{div}_{\mathrm{h}} g v=0, \\
& \left.v\right|_{t=0}=v_{0} .
\end{aligned}
$$


We notice that, already in 1994, Ciuperca [4] has derived the linear version of (1.8) in connection with the Stokes problem and has proved a convergence result for the linear problem. Also Roh [36] has studied the above limit equation in the particular case where $\nabla g$ is small. Note also that the limit operator $v \mapsto \operatorname{div}_{\mathrm{h}}(g v)$ of the divergence already appeared in other works dealing with the Euler equations ([19] for example).

In Theorem 6.1 of Section 6 , we shall prove a general convergence result, which contains the following Theorem 1.2 as a corollary. We denote by $V_{0}$ (respectively $\left.H_{0}\right)$ ) the subspace of vectors in $H^{1}\left(\mathbb{T}^{2}\right)^{2}$ (respectively in $L^{2}\left(\mathbb{T}^{2}\right)^{2}$ ) corresponding to the "limit" of the space $V_{\varepsilon}$ (respectively $H_{\varepsilon}$ ) at $\varepsilon=0$ (for the precise definition of $V_{0}$ and $H_{0}$ ), we refer to Section 6). As for the classical 2D Navier-Stokes equations, one shows in Section 6 that, for any $v_{0} \in H_{0}$, there exists a unique strong solution $v(t) \in C^{0}\left([0,+\infty), H_{0}\right) \cap C^{0}\left((0,+\infty), V_{0}\right) \cap$ $L^{\infty}\left((\delta,+\infty), V_{0}\right)$ of the equations (1.8), where $\delta$ is any positive number.

Theorem 1.2. Let $u_{0}^{\varepsilon} \in V_{\varepsilon}$ and $f_{\varepsilon} \in L^{\infty}\left((0, \infty), L^{2}\left(\Omega_{\varepsilon}\right)^{3}\right)$ be a family of initial data and forcing terms satisfying the hypotheses (1.5). We assume moreover that there exist a twodimensional vector $v_{0} \in H_{0}$ and a two-dimensional forcing term $f_{0} \in L^{\infty}\left((0, \infty), L^{2}\left(\mathbb{T}^{2}\right)^{2}\right)$ such that

$$
\begin{aligned}
& \lim _{\varepsilon \rightarrow 0} M_{h} f_{\varepsilon}=\left(f_{0}, 0\right) \quad \text { in } L^{\infty}\left((0, \infty), L^{2}\left(\mathbb{T}^{2}\right)^{3}\right), \\
& \lim _{\varepsilon \rightarrow 0} \varepsilon^{1 / 2}\left(I-M_{h}\right) f_{\varepsilon}=0 \quad \text { in } L^{\infty}\left((0, \infty), L^{2}\left(\Omega_{\varepsilon}\right)^{3}\right), \\
& \lim _{\varepsilon \rightarrow 0} M_{h} u_{0}^{\varepsilon}=\left(v_{0}, 0\right) \quad \text { in } L^{2}\left(\mathbb{T}^{2}\right)^{3}, \quad \lim _{\varepsilon \rightarrow 0} \varepsilon^{1 / 2} u_{0}^{\varepsilon}=0 \quad \text { in } H^{1}\left(\Omega_{\varepsilon}\right)^{3},
\end{aligned}
$$

then, for each $T>0$, we have the convergence result

$$
\lim _{\varepsilon \rightarrow 0} M_{h} u^{\varepsilon}(t)=(v(t), 0) \quad \text { in } C^{0}\left([0, T], L^{2}\left(\mathbb{T}^{2}\right)^{3}\right)
$$

where $u^{\varepsilon}(t)$ (respectively $v(t)$ ) is the solution of the equations (1.2)-(1.3) (respectively (1.8)) with initial data $u_{0}^{\varepsilon}$ (respectively $v_{0}$ ).

The paper is organized as follows. Section 2 contains the precise formulation of the additional conditions made on the forcing term and the initial data, that is, the conditions $(\mathrm{H})$ and $\left(\mathrm{H}_{f}\right)$. We also discuss there a uniform Korn inequality and introduce the linear Stokes operator $A_{\varepsilon}$. In Section 3, we prove all the auxiliary estimates, which are necessary in order to bound the nonlinear part in (1.2). Section 4 is devoted to the precise estimates of the quadratic term in equation (1.2). In Section 5, we prove the global existence result stated in Theorem 1.1. In Section 6, we assume that the forcing term $f=f_{\varepsilon}$ in (1.2) depends on $\varepsilon$ and that $M_{h} f_{\varepsilon}$ converges, in the sense of (6.1), to a two-dimensional function $f_{0}$, which allows us to define the limiting equation (6.5) and to compare the solutions of (1.2) with those of the limit equation (see Theorem 1.2). Finally, in Section 7, we show that the dynamical system generated by the equations (1.2) admits a (local) attractor $\mathcal{A}_{\varepsilon}$ in $V_{\varepsilon}$. We prove that $\mathcal{A}_{\varepsilon}$ is in fact the global attractor of all the weak Leray-Hopf solutions of (1.2). We also compare this attractor $\mathcal{A}_{\varepsilon}$ with the global attractor $\mathcal{A}_{0}$ of the limiting equations (6.2) defined in Section 6. 


\section{Formulation of the problem}

2.1. The uniform Korn inequality. As we already indicated, an additional hypothesis, other than regularity, has to be assumed on the data. For example, if $g$ is constant, then we will see that the limit problem is the bidimensional periodic Navier-Stokes equation and therefore requires the assumption that the first two components of the velocity have vanishing mean. Technically, the need for this kind of assumption appears in the following way. According to the Green formula (2.17), when making energy estimates on (1.2), we are led to use the quantity

$$
E(u, u)=4\|D(u)\|_{L^{2}}^{2}, \quad \text { where }[D(u)]_{i, j}=\frac{1}{2}\left(\partial_{i} u_{j}+\partial_{j} u_{i}\right), \quad \text { for } 1 \leq i, j \leq 3,
$$

is the deformation tensor. What is needed is that $E(u, u)$ be equivalent to the square of the $H^{1}$-norm of $u$ on a suitable function space $H_{\varepsilon}$. A short well-known computation shows that

$$
E(U, U)=0 \quad \Longleftrightarrow \quad D(U)=0 \quad \Longleftrightarrow \quad U=A+B \times x,
$$

where $A=\left(a_{1}, a_{2}, a_{3}\right)$ and $B=\left(b_{1}, b_{2}, b_{3}\right)$ are vectors that do not depend on $x \in \Omega_{\varepsilon}$. This property implies that $\sqrt{E(u(t), u(t))}$ cannot be equivalent to the $H^{1}$-norm of $u(t)$ if $u(t)=A(t)+B(t) \times x$. Thus we need at least to insure that nonzero initial data and nonzero solutions $u(t)$ cannot be of this form. This means that we have to impose some restrictions on the data $\left(u_{0}, f\right)$.

Suppose now that $U=A+B \times x$ verifies the boundary conditions (1.3). Since $U \cdot N=0$ on $\Gamma_{0} \cup \Gamma_{\varepsilon}$, we find that $a_{3}=b_{1}=b_{2}=0$ and $a_{1} \partial_{1} g+a_{2} \partial_{2} g=0$. Due to the periodicity of $U$, one has $b_{3}=0$. Thus, with the above boundary conditions, $E(U, U)=0$, for $U \neq 0$, if and only if there are constants $a_{1}, a_{2}$ (with $a_{1}^{2}+a_{2}^{2} \neq 0$ ) such that $U=\left(a_{1}, a_{2}, 0\right)$ and $a_{1} \partial_{1} g+a_{2} \partial_{2} g=0$. To make sure that such a vector field cannot be an initial datum, we make the following assumption on the initial data:

$$
a_{1} \int_{\Omega_{\varepsilon}} u_{1}(0, x) d x+a_{2} \int_{\Omega_{\varepsilon}} u_{2}(0, x) d x=0
$$

for all constants $a_{1}, a_{2}$ such that $a_{1} \partial_{1} g+a_{2} \partial_{2} g \equiv 0$.

Moreover, we require that this assumption $(\mathrm{H})$ be propagated by the equation. In other words, if $u(0)$ satisfies $(\mathrm{H})$, then the solution $u(t)$ satisfies $(\mathrm{H})$, for all time $t$, as well. As we now show, this is accomplished under the following additional condition on the forcing term $f=\left(f_{1}, f_{2}, f_{3}\right)$ :

$\left(\mathrm{H}_{f}\right) \quad$ for all $t \geq 0$, one has

$$
\begin{aligned}
& a_{1} \int_{\Omega_{\varepsilon}} f_{1}(t, x) d x+a_{2} \int_{\Omega_{\varepsilon}} f_{2}(t, x) d x=0, \\
& \quad \text { for all constants } a_{1}, a_{2} \text { such that } a_{1} \partial_{1} g+a_{2} \partial_{2} g \equiv 0 .
\end{aligned}
$$

Indeed, let us fix a forcing term $f$ satisfying the condition $\left(\mathrm{H}_{f}\right)$. Let $u_{0}$ be initial data satisfying the additional condition $(\mathrm{H})$ and $u=u(t)$ be the corresponding local solution of the Navier-Stokes equations (1.2). If $a_{1}, a_{2}$ are constants such that $a_{1} \partial_{1} g+a_{2} \partial_{2} g \equiv 0$, then the vector $A=\left(a_{1}, a_{2}, 0\right)$ satisfies the boundary condition $A \cdot N=0$ on the boundary $\Gamma_{0} \cup \Gamma_{\varepsilon}$. Taking the inner product in $L^{2}\left(\Omega_{\varepsilon}\right)^{3}$ of (1.2) with the vector $A$ and applying the 
Green formula (2.17), we obtain

$$
\frac{d}{d t} \int_{\Omega_{\varepsilon}} u \cdot A d x+\int_{\Omega_{\varepsilon}} u \cdot \nabla u \cdot A d x=0 .
$$

Integrating the term $\int_{\Omega_{\varepsilon}} u \cdot \nabla u \cdot A d x$ by parts and using the facts that div $u=0$ in $\Omega_{\varepsilon}$ and that $u \cdot N=0$ on the boundary $\Gamma_{0} \cup \Gamma_{\varepsilon}$, we easily check that

$$
\int_{\Omega_{\varepsilon}} u \cdot \nabla u \cdot A d x=0
$$

and thus that, for any $t \geq 0$,

$$
\int_{\Omega_{\varepsilon}} u(t, x) \cdot A d x=0
$$

which means that the condition $(\mathrm{H})$ is positively invariant under the semiflow generated by the Navier-Stokes equations (1.2) provided that the forcing term verifies the $\left(\mathrm{H}_{f}\right)$ hypothesis.

Remark 2.1. The conditions $(\mathrm{H})$ and $\left(\mathrm{H}_{f}\right)$ can be made more explicit by considering three typical cases:

(a) There are no constants $a_{1}, a_{2}$ (with $a_{1}^{2}+a_{2}^{2} \neq 0$ ) such that $a_{1} \partial_{1} g+a_{2} \partial_{2} g=0$; in this case the hypotheses $(\mathrm{H})$ and $\left(\mathrm{H}_{f}\right)$ are empty.

(b) The function $g$ is constant; in this case $a_{1}$ and $a_{2}$ are arbitrary and (H) becomes $\int_{\Omega_{\varepsilon}} u_{1}(0, x) d x=\int_{\Omega_{\varepsilon}} u_{2}(0, x) d x=0$.

(c) The function $g$ is not constant but there exist constants $a_{1}, a_{2}$ (with $a_{1}^{2}+a_{2}^{2} \neq 0$ ) such that $a_{1} \partial_{1} g+a_{2} \partial_{2} g=0$. In this case we note that every pair of constants $\left(a_{1}^{\prime}, a_{2}^{\prime}\right)$ such that $a_{1}^{\prime} \partial_{1} g+a_{2}^{\prime} \partial_{2} g=0$ is necessarily a multiple of $\left(a_{1}, a_{2}\right)$ and therefore $(\mathrm{H})$ reduces to one condition: $a_{1} \int_{\Omega_{\varepsilon}} u_{1}(0, x) d x+a_{2} \int_{\Omega_{\varepsilon}} u_{2}(0, x) d x=0$. We also note that in this case, the function $g$ must necessarily be of the form $g\left(x_{1}, x_{2}\right)=g^{*}\left(a_{2} x_{1}-a_{1} x_{2}\right)$ where $g^{*}$ is a periodic function of class $C^{3}$.

The aim of the remainder of this section is to prove that hypothesis $(\mathrm{H})$ is sufficient for our purposes, namely that a Korn inequality holds with positive constants independent of $\varepsilon$. Because the constants are independent of $\varepsilon$, for $0<\varepsilon \leq 1$, we will refer to this inequality as the uniform Korn inequality. We now define the space

$$
\begin{array}{r}
\mathcal{H}^{1}=\left\{U \in H^{1}\left(\Omega_{\varepsilon}\right)^{3} \mid U \text { is periodic in } x_{1} \text { and } x_{2}, U \cdot N=0 \text { on } \Gamma_{\varepsilon} \cup \Gamma_{0},\right. \\
U \text { satisfies the condition }(\mathrm{H})\} .
\end{array}
$$

We note that the unit outward normal $N$ to $\Gamma_{\varepsilon}$ is given by

$$
N=d_{\varepsilon}^{-1}\left(-\varepsilon \partial_{1} g,-\varepsilon \partial_{2} g, 1\right), \quad \text { where } d_{\varepsilon}=d_{\varepsilon}\left(x_{h}\right)=\sqrt{1+\varepsilon^{2}|\nabla g|^{2}} .
$$

We remark that the space $V_{\varepsilon}$ defined in the Introduction is given by

$$
V_{\varepsilon}=\mathcal{H}^{1} \cap\left\{U \in H^{1}\left(\Omega_{\varepsilon}\right)^{3} \mid \operatorname{div} U=0\right\} .
$$

We also introduce the bilinear form $E(\cdot, \cdot)$ on $\mathcal{H}^{1} \times \mathcal{H}^{1}$ as follows,

$E\left(U, U^{*}\right)=4 \int_{\Omega_{\varepsilon}} D(U) \cdot D\left(U^{*}\right)=\sum_{i, j} \int_{\Omega_{\varepsilon}}\left(\partial_{i} U_{j}+\partial_{j} U_{i}\right)\left(\partial_{i} U_{j}^{*}+\partial_{j} U_{i}^{*}\right), \quad$ for all $U, U^{*} \in \mathcal{H}^{1}$. 
We now follow the argument of [41], which is given in the case of a fixed domain, to prove the following result.

Theorem 2.1 (Uniform Korn Inequality). There exist positive constants $c_{0}$, $c_{0}^{*}$ such that, for any $\varepsilon \in(0,1]$,

$$
c_{0}\|U\|_{H^{1}}^{2} \leq E(U, U) \leq c_{0}^{*}\|U\|_{H^{1}}^{2}, \quad \text { for any } U \in \mathcal{H}^{1} .
$$

Proof. Since $E(U, U) \leq 4\|\nabla U\|_{L^{2}}^{2}$, the upper bound in (2.2) holds with $c_{0}^{*}=4$. We shall show the lower bound, by proving first that there exists a positive constant $c$ such that, for $\varepsilon \in(0,1], U \in \mathcal{H}^{1}$,

$$
c\|\nabla U\|_{L^{2}}^{2} \leq E(U, U) .
$$

Integrating the expression $\int_{\Omega_{\varepsilon}} \partial_{i} U_{j} \partial_{j} U_{i} d x$ twice by parts and using the fact that $U \cdot N$ vanishes on the boundary $\Gamma_{0} \cup \Gamma_{\varepsilon}$, we obtain that

$$
E(U, U)=2\|\nabla U\|_{L^{2}}^{2}+2 \int_{\Omega_{\varepsilon}}(\operatorname{div} U)^{2} d x+2 \sum_{i, j=1}^{3} \int_{\Gamma_{\varepsilon}} U_{j} \partial_{j} U_{i} N_{i} d \sigma_{x} .
$$

In the expression above, we also used the fact that $U_{3}=N_{1}=N_{2}=0$ on $\Gamma_{0}$ to deduce that $U_{j} \partial_{j} U_{i} N_{i}$ vanishes on $\Gamma_{0}$. Next, since $U$ is tangent to the boundary, we have that

$$
U_{3}\left(x_{h}, \varepsilon g\left(x_{h}\right)\right)=\varepsilon \partial_{1} g\left(x_{h}\right) U_{1}\left(x_{h}, \varepsilon g\left(x_{h}\right)\right)+\varepsilon \partial_{2} g\left(x_{h}\right) U_{2}\left(x_{h}, \varepsilon g\left(x_{h}\right)\right) .
$$

The above equality only holds on the boundary $\Gamma_{\varepsilon}$. This implies that we can differentiate it only with respect to the horizontal variables. Differentiating the term $U_{3}\left(x_{h}, \varepsilon g\left(x_{h}\right)\right)$, given in (2.5), with respect to $x_{i}$ and multiplying the resulting expression by $U_{i}$, for $i=1,2$, we get

$$
\begin{aligned}
& \sum_{i=1}^{2}\left[U_{i} \partial_{i} U_{3}+\varepsilon \partial_{i} g U_{i} \partial_{3} U_{3}\right]\left(x_{h}, \varepsilon g\left(x_{h}\right)\right)= \\
& \qquad \sum_{i=1}^{2} U_{i}\left(x_{h}, \varepsilon g\left(x_{h}\right)\right) \partial_{i}\left[\varepsilon \partial_{1} g\left(x_{h}\right) U_{1}\left(x_{h}, \varepsilon g\left(x_{h}\right)\right)+\varepsilon \partial_{2} g\left(x_{h}\right) U_{2}\left(x_{h}, \varepsilon g\left(x_{h}\right)\right)\right] .
\end{aligned}
$$

We note that, due to the equality (2.5), the left-hand side member of the previous equality can be written as

$$
\sum_{i=1}^{2}\left[U_{i} \partial_{i} U_{3}+\varepsilon \partial_{i} g U_{i} \partial_{3} U_{3}\right]\left(x_{h}, \varepsilon g\left(x_{h}\right)\right)=\left[U_{1} \partial_{1} U_{3}+U_{2} \partial_{2} U_{3}+U_{3} \partial_{3} U_{3}\right]\left(x_{h}, \varepsilon g\left(x_{h}\right)\right) \text {. }
$$

Computing explicitly the derivatives of the right-hand side member of the equality (2.6) and using several times the equality (2.5) (in the same way as in the equality (2.7)), we deduce from (2.6) and (2.7), the following equality,

$$
\begin{aligned}
\left(U_{1} \partial_{1} U_{3}+U_{2} \partial_{2} U_{3}+\right. & \left.U_{3} \partial_{3} U_{3}\right)\left(x_{h}, \varepsilon g\left(x_{h}\right)\right)=\left[2 \varepsilon \partial_{1} \partial_{2} g U_{1} U_{2}+\varepsilon^{2} \partial_{1} g \partial_{2} g\left(U_{2} \partial_{3} U_{1}+U_{1} \partial_{3} U_{2}\right)\right. \\
& +\varepsilon \partial_{1} g\left(U_{1} \partial_{1} U_{1}+U_{2} \partial_{2} U_{1}\right)+\varepsilon \partial_{2} g\left(U_{2} \partial_{2} U_{2}+U_{1} \partial_{1} U_{2}\right)+\varepsilon \partial_{1}^{2} g U_{1}^{2} \\
& \left.+\varepsilon \partial_{2}^{2} g U_{2}^{2}+\varepsilon^{2}\left(\partial_{1} g\right)^{2} U_{1} \partial_{3} U_{1}+\varepsilon^{2}\left(\partial_{2} g\right)^{2} U_{2} \partial_{3} U_{2}\right]\left(x_{h}, \varepsilon g\left(x_{h}\right)\right) .
\end{aligned}
$$


We now want to compute the integral term $\sum_{i, j=1}^{3} \int_{\Gamma_{\varepsilon}} U_{j} \partial_{j} U_{i} N_{i}(x) d \sigma_{x}$. Replacing the term $\sum_{j=1}^{3} U_{j} \partial_{j} U_{3}\left(x_{h}, \varepsilon g\left(x_{h}\right)\right)$ (respectively $U_{3}\left(x_{h}, \varepsilon g\left(x_{h}\right)\right)$ ) by its expression given in (2.8) (respectively in (2.5)) and using the explicit values of $N_{i}$ (see (2.1)), we readily obtain that

$$
\sum_{i, j=1}^{3} \int_{\Gamma_{\varepsilon}} U_{j} \partial_{j} U_{i} N_{i}(x) d \sigma_{x}=\int_{\Gamma_{\varepsilon}} \frac{\varepsilon}{d_{\varepsilon}}\left(U_{1}^{2} \partial_{1}^{2} g+U_{2}^{2} \partial_{2}^{2} g+2 U_{1} U_{2} \partial_{1} \partial_{2} g\right) d \sigma_{x}
$$

where $d_{\varepsilon}=\sqrt{1+\varepsilon^{2}|\nabla g|^{2}}$. We deduce from (2.4) and (2.9) that

$$
\|\nabla U\|_{L^{2}}^{2} \leq \frac{1}{2} E(U, U)+c(g) \varepsilon \int_{\Gamma_{\varepsilon}}\left(U_{1}^{2}+U_{2}^{2}\right) d \sigma_{x},
$$

where $c(g)$ is a positive constant depending only on $g$. We set $\delta=\frac{1}{2 c(g)}$.

To prove the inequality (2.3), we are now going to show that there exists a positive constant $K$ (independent of $\varepsilon$ ) such that, for $0<\varepsilon \leq 1$,

$$
\varepsilon \int_{\Gamma_{\varepsilon}}\left(U_{1}^{2}+U_{2}^{2}\right) d \sigma_{x} \leq \delta\|\nabla U\|_{L^{2}}^{2}+K E(U, U), \quad \text { for all } U \in \mathcal{H}^{1} .
$$

According to the proof of Lemma 4 of [41], we already know that the inequality $(2.10)$ holds for some positive constant $K_{\varepsilon}$. However, this constant $K_{\varepsilon}$ could depend on $\varepsilon$. Here we prove by contradiction that this constant does not tend to infinity when $\varepsilon$ goes to zero. If (2.10) does not hold with a constant $K$ independent of $\varepsilon$, then, for each $n \in \mathbf{N}$, there exist $\varepsilon_{n}>0$ and a vector $U_{n}^{*} \in \mathcal{H}^{1}$ such that

$$
\varepsilon_{n} \int_{\Gamma_{\varepsilon_{n}}}\left(\left(U_{n, 1}^{*}\right)^{2}+\left(U_{n, 2}^{*}\right)^{2}\right) d \sigma_{x} \geq \delta\left\|\nabla U_{n}^{*}\right\|_{L^{2}}^{2}+n E\left(U_{n}^{*}, U_{n}^{*}\right)
$$

and $\varepsilon_{n}$ converges to zero when $n$ goes to infinity. Replacing the vector $U_{n}^{*}$ by

$$
U_{n}=U^{*}\left(\int_{\Gamma_{\varepsilon_{n}}}\left(\left(U_{n, 1}^{*}\right)^{2}+\left(U_{n, 2}^{*}\right)^{2}\right) d \sigma_{x}\right)^{-1 / 2},
$$

we claim that, for each $n \in \mathbf{N}$, there exist $\varepsilon_{n}>0$ and a vector $U_{n} \in \mathcal{H}^{1}$ such that

$$
\varepsilon_{n} \geq \delta\left\|\nabla U_{n}\right\|_{L^{2}}^{2}+n E\left(U_{n}, U_{n}\right), \quad \int_{\mathbb{T}^{2}}\left(\left(U_{n, 1}\right)^{2}+\left(U_{n, 2}\right)^{2}\right)\left(x_{h}, \varepsilon g\right) \sqrt{1+\varepsilon^{2}|\nabla g|^{2}} d x_{h}=1 .
$$

In order to study the limit of the sequence $U_{n}$ when $n$ goes to infinity, we perform a change of variables which transforms the domain $\Omega_{\varepsilon}$ into the fixed domain $Q=\Omega_{1}$. More precisely, we set $y_{3}=\varepsilon^{-1} x_{3}$ and

$$
U_{n}\left(x_{1}, x_{2}, x_{3}\right)=U_{n}\left(y_{1}, y_{2}, \varepsilon y_{3}\right)=\widetilde{u}_{n}\left(y_{1}, y_{2}, y_{3}\right), \quad \text { for all }\left(y_{1}, y_{2}, y_{3}\right) \in \Omega_{1} .
$$

This implies that

$$
\frac{\partial U_{n}}{\partial x_{i}}=\frac{\partial \widetilde{u}_{n}}{\partial y_{i}}, \text { for } i=1,2 \text {, and } \frac{\partial U_{n}}{\partial x_{3}}=\frac{1}{\varepsilon} \frac{\partial \widetilde{u}_{n}}{\partial y_{3}}
$$


We introduce the "scaled" operator $\nabla_{\varepsilon}=\left(\frac{\partial}{\partial y_{1}}, \frac{\partial}{\partial y_{2}}, \varepsilon^{-1} \frac{\partial}{\partial y_{3}}\right)$ together with the "scaled" bilinear form

$$
E_{\varepsilon}\left(u^{1}, u^{2}\right)=\int_{\Omega_{1}}\left(\sum_{i, j=1}^{3}\left(\varepsilon^{-\delta_{j, 3}} \frac{\partial u_{i}^{1}}{\partial y_{j}}+\varepsilon^{-\delta_{i, 3}} \frac{\partial u_{j}^{1}}{\partial y_{i}}\right) \cdot\left(\varepsilon^{-\delta_{j, 3}} \frac{\partial u_{i}^{2}}{\partial y_{j}}+\varepsilon^{-\delta_{i, 3}} \frac{\partial u_{j}^{2}}{\partial y_{i}}\right)\right) d y,
$$

where $\delta_{j, 3}$ is the Kronecker symbol. The properties (2.11) then become

$$
\begin{aligned}
& 1 \geq \delta\left\|\nabla_{\varepsilon_{n}} \widetilde{u}_{n}\right\|_{L^{2}\left(\Omega_{1}\right)}^{2}+n E_{\varepsilon_{n}}\left(\widetilde{u}_{n}, \widetilde{u}_{n}\right), \\
& \int_{\mathbb{T}^{2}}\left(\left(\widetilde{u}_{n, 1}\right)^{2}+\left(\widetilde{u}_{n, 2}\right)^{2}\right)\left(y_{h}, g\right) \sqrt{1+\varepsilon^{2}|\nabla g|^{2}} d y_{h}=1 .
\end{aligned}
$$

We deduce from the above relations that $\nabla \widetilde{u}_{n}$ is bounded in $L^{2}\left(\Omega_{1}\right)$. We next decompose $\widetilde{u}_{n, i}, i=1,2$, into the sum of his total mean value and a vector with vanishing total mean value, that is, we write $\widetilde{u}_{n, i}=\widetilde{w}_{n, i}+c_{n, i}$, where $c_{n, i}=\int_{\Omega_{1}} \widetilde{u}_{n, i}(y) d y$. Since the vector $\widetilde{w}_{n, i}$ is uniformly bounded in $H^{1}\left(\Omega_{1}\right)$, its trace on $\Gamma_{1}$ is uniformly bounded in $L^{2}\left(\Gamma_{1}\right)$. Therefore the second property in (2.12) shows that $c_{n, i}, i=1,2$, is uniformly bounded in $n$ and $\varepsilon$. Moreover, $\widetilde{u}_{n, 3}$ vanishes on the lower part of the boundary $\Gamma_{0}$. Therefore, $\widetilde{u}_{n}$ is bounded in $H^{1}\left(\Omega_{1}\right)$. This implies that there exists a subsequence, which we relabel as $\widetilde{u}_{n}$, such that $\widetilde{u}_{n}$ converges weakly to $\widetilde{v}$ in $\left(H^{1}(Q)\right)^{3}$. The inequality relation in (2.12) implies in particular that

$$
\frac{1}{\varepsilon_{n}}\left\|\frac{\partial \widetilde{u}_{n}}{\partial y_{3}}\right\|_{L^{2}\left(\Omega_{1}\right)} \leq \frac{1}{\sqrt{\delta}} \text { and } \frac{1}{\varepsilon_{n}}\left\|\frac{\partial \widetilde{u}_{n, 3}}{\partial y_{3}}\right\|_{L^{2}\left(\Omega_{1}\right)} \leq \frac{1}{\sqrt{n}} .
$$

We deduce from (2.13) that the limit $\widetilde{v}$ is independent of $y_{3}$, that is, $\widetilde{v}$ belongs to $\left(H^{1}\left(\mathbb{T}^{2}\right)\right)^{3}$. The boundary condition $\widetilde{u}_{n, 3}\left(x_{1}, x_{2}, 0\right)=0$ implies that, at the limit, $\widetilde{v}_{3}\left(x_{1}, x_{2}, 0\right)=0$ and thus that $\widetilde{v}\left(y_{1}, y_{2}\right)=\left(\widetilde{v}_{1}\left(y_{1}, y_{2}\right), \widetilde{v}\left(y_{1}, y_{2}\right), 0\right)$. Using the property (2.13) together with the fact that $\widetilde{u}_{n, 3}\left(y_{1}, y_{2}, 0\right)=0$, we at once show that

$$
\frac{1}{\varepsilon_{n}}\left\|\widetilde{u}_{n, 3}\right\|_{L^{2}\left(\Gamma_{1}\right)} \leq \frac{C}{\sqrt{n}},
$$

where $C$ is a positive constant independent of $n$ and $\varepsilon_{n}$. The boundary condition satisfied by $\widetilde{u}_{n}$ on $\Gamma_{1}$ assumes the form

$$
\begin{aligned}
\frac{1}{\varepsilon_{n}} \widetilde{u}_{n, 3}\left(y_{1}, y_{2}, g\left(y_{1}, y_{2}\right)\right) & -\partial_{1} g\left(y_{1}, y_{2}\right) \widetilde{u}_{n, 1}\left(y_{1}, y_{2}, g\left(y_{1}, y_{2}\right)\right) \\
& -\partial_{2} g\left(y_{1}, y_{2}\right) \widetilde{u}_{n, 2}\left(y_{1}, y_{2}, g\left(y_{1}, y_{2}\right)\right)=0,
\end{aligned}
$$

from which we deduce, together with (2.14) that at the limit,

$$
\partial_{1} g\left(y_{1}, y_{2}\right) \widetilde{v}_{1}\left(y_{1}, y_{2}\right)+\partial_{2} g\left(y_{1}, y_{2}\right) \widetilde{v}_{2}\left(y_{1}, y_{2}\right)=0 \text {. }
$$

From the property $E_{\varepsilon_{n}}\left(\widetilde{u}_{n}, \widetilde{u}_{n}\right) \leq \frac{1}{n}$, we also deduce that $E(\widetilde{v}, \widetilde{v})=0$. Therefore, since $\widetilde{v}$ satisfies the "boundary condition" (2.15), there exist constants $a_{1}, a_{2}$ such that $a_{1} \partial_{1} g+$ $a_{2} \partial_{2} g=0$ and $\widetilde{v}=\left(a_{1}, a_{2}, 0\right)$. But the condition (H) implies that $a_{1} \widetilde{v}_{1}+a_{2} \widetilde{v}_{2}=0$, which yields $\widetilde{v}=0$. The equality relation in (2.12) together with the strong convergence of $\widetilde{u}_{n}$ towards $\widetilde{v}$ in $L^{2}\left(\Gamma_{1}\right)$ imply that $\int_{\mathbb{T}^{2}}\left(\widetilde{v}_{1}^{2}+\widetilde{v}_{2}^{2}\right) d y_{h}=1$, which contradicts the fact that $\widetilde{v}=0$. We thus have proved the inequality (2.3). 
It remains to prove that there exist a positive constant $c$ such that, for $0<\varepsilon \leq 1$, for any $U \in \mathcal{H}^{1}$,

$$
c\|U\|_{L^{2}}^{2} \leq E(U, U) .
$$

To show this inequality, one uses the same type of argument by contradiction as above. As here the contradiction argument is easier and does not contain any novel feature, we do not present the details.

Remark 2.2. It follows from the inequality (2.16) that

$$
c\|U\|_{L^{2}}^{2} \leq E(U, U) \leq 4\|\nabla U\|_{L^{2}}^{2}, \text { for all } U \in \mathcal{H}^{1} .
$$

In other words, the Poincaré inequality holds for any $U$ in $\mathcal{H}^{1}$.

We next give a simple Green formula in connection with the Navier boundary conditions. This formula has already been given in [41] and [46].

Lemma 2.2. Let $\widetilde{u}$ and $\bar{u}$ be vectors in $H^{2}\left(\Omega_{\varepsilon}\right)^{3}$ and in $H^{1}\left(\Omega_{\varepsilon}\right)^{3}$ respectively. Then one has the identity:

$$
\int_{\Omega_{\varepsilon}} \triangle \widetilde{u} \cdot \bar{u}=-\frac{1}{2} E(\widetilde{u}, \bar{u})+\int_{\Omega_{\varepsilon}} \operatorname{div} \widetilde{u} \operatorname{div} \bar{u}+\int_{\partial \Omega_{\varepsilon}}\{2[D(\widetilde{u}) N] \cdot \bar{u}-\operatorname{div} \widetilde{u} \bar{u} \cdot N\} .
$$

Proof. The proof is a simple integration by parts. One writes

$$
\begin{aligned}
\int_{\Omega_{\varepsilon}} \Delta \widetilde{u} \cdot \bar{u}= & \sum_{i, j} \int_{\Omega_{\varepsilon}} \partial_{i}\left(\partial_{i} \widetilde{u}_{j}+\partial_{j} \widetilde{u}_{i}\right) \bar{u}_{j}-\sum_{j} \int_{\Omega_{\varepsilon}} \partial_{j} \operatorname{div} \widetilde{u} \bar{u}_{j} \\
= & -\sum_{i, j} \int_{\Omega_{\varepsilon}}\left(\partial_{i} \widetilde{u}_{j}+\partial_{j} \widetilde{u}_{i}\right) \partial_{i} \bar{u}_{j}+\sum_{i, j} \int_{\partial \Omega_{\varepsilon}}\left(\partial_{i} \widetilde{u}_{j}+\partial_{j} \widetilde{u}_{i}\right) N_{i} \bar{u}_{j} \\
& +\int_{\Omega_{\varepsilon}} \operatorname{div} \widetilde{u} \operatorname{div} \bar{u}-\int_{\partial \Omega_{\varepsilon}} \operatorname{div} \widetilde{u} \bar{u} \cdot N \\
= & -\frac{1}{2} E(\widetilde{u}, \bar{u})+\int_{\Omega_{\varepsilon}} \operatorname{div} \widetilde{u} \operatorname{div} \bar{u}+\int_{\partial \Omega_{\varepsilon}}\{2[D(\widetilde{u}) N] \cdot \bar{u}-\operatorname{div} \widetilde{u} \bar{u} \cdot N\} .
\end{aligned}
$$

2.2. The Stokes operator. Since by Theorem 2.1, the bilinear form $E(\cdot, \cdot)$ is a coercive continuous symmetric bilinear form on the space $V_{\varepsilon}$, one can show, by using the LaxMilgram theorem together with the Riesz representation theorem that there exists a (sectorial) self-adjoint positive operator $A_{\varepsilon}: D\left(A_{\varepsilon}\right) \rightarrow H_{\varepsilon}$, where $D\left(A_{\varepsilon}\right)=\left\{U \in V_{\varepsilon} \mid A_{\varepsilon} U \in H_{\varepsilon}\right\}$ such that, for all $U \in D\left(A_{\varepsilon}\right)$,

$$
\left(A_{\varepsilon} U, U^{*}\right)_{L^{2}}=\frac{1}{2} E\left(U, U^{*}\right), \quad \text { for all } U^{*} \in V_{\varepsilon},
$$

(see [20], [42] or [43]). This operator $A_{\varepsilon}$ is called the Stokes operator. We notice that $V_{\varepsilon}=D\left(A_{\varepsilon}^{1 / 2}\right)$. Since $A_{\varepsilon}$ is self-adjoint, the following equality holds

$$
\left(A_{\varepsilon} U, U^{*}\right)_{L^{2}}=\left(A_{\varepsilon}^{1 / 2} U, A_{\varepsilon}^{1 / 2} U^{*}\right)_{L^{2}}, \quad \text { for any } U \in D\left(A_{\varepsilon}\right), \quad U^{*} \in V_{\varepsilon},
$$


and, in particular,

$$
\frac{1}{2} E(U, U)=\left\|A_{\varepsilon}^{1 / 2} U\right\|_{L^{2}}^{2}, \quad \text { for any } U \in D\left(A_{\varepsilon}\right) .
$$

In [41], the following regularity result has been proved

$$
D\left(A_{\varepsilon}\right)=\left\{U \in H^{2}\left(\Omega_{\varepsilon}\right)^{3} \cap V_{\varepsilon} ;\left.[D(u) N]_{t a n}\right|_{\Gamma_{0} \cup \Gamma_{\varepsilon}}=0\right\} .
$$

In view of Lemma 2.2 and of this regularity result,

$$
A_{\varepsilon} U=-\mathbb{P}_{\varepsilon} \Delta U, \quad \text { for all } U \in D\left(A_{\varepsilon}\right) .
$$

This implies in particular that the equality $A_{\varepsilon}=-\mathbb{P}_{\varepsilon} \Delta U$ can be given as an alternate definition of the Stokes operator.

2.3. The nonlinear problem. Let $f$ be a forcing term in $L^{\infty}\left((0, \infty), L^{2}\left(\Omega_{\varepsilon}\right)^{3}\right)$ satisfying the condition $\left(\mathrm{H}_{f}\right)$. As in the case of classical boundary conditions, one shows that, for any $u_{0} \in V_{\varepsilon}$, there exists a unique maximally defined strong local solution $u=u(t) \in$ $C^{0}\left([0, T), V_{\varepsilon}\right)$ of the equations (1.2)-(1.3) with $u(0)=u_{0}$, where $T=T\left(u_{0}, f\right)$ depends only on the data $u_{0}$ and $f$. Furthermore, this solution satisfies

$$
u \in L_{l o c}^{2}\left((0, T), D\left(A_{\varepsilon}\right)\right) \cap H_{l o c}^{1}\left((0, T), H_{\varepsilon}\right) .
$$

Moreover, $u$ satisfies the equations (1.2) for almost every $t$ in the sense of distributions in the spatial variable. We remark that $u$ is also a mild solution of the abstract evolutionary equation associated to (1.2)-(1.3):

$$
\partial_{t} u+A_{\varepsilon} u+\mathbb{P}_{\varepsilon}(u \cdot \nabla u)=\mathbb{P}_{\varepsilon} f, \quad u(0)=u_{0} .
$$

It is worthwhile to remark that, if $f$ is locally Lipschitz in time with values in $L^{2}\left(\Omega_{\varepsilon}\right)^{3}$, the above solution $u$ is a classical solution (in the sense of evolutionary equations), that is,

$$
u \in C^{0}\left((0, T), D\left(A_{\varepsilon}\right)\right) \cap C^{1}\left((0, T), H_{\varepsilon}\right),
$$

and $u=u(t)$ satisfies the equations (1.2) for every $t$ in the sense of distributions in the spatial variable.

As in the case of classical boundary conditions, the existence result can be proved mainly in two ways. Since $E(\cdot, \cdot)$ is a positive definite bilinear form, we can apply the classical Galerkin method (also called Bubnov-Galerkin method), which roughly speaking consists in approximating the Navier-Stokes equations by a sequence of finite dimensional-problems and to prove the convergence of the solutions of these approximate problems towards a solution of the Equations (1.2) (see for example [21], [42], [6] or [39]).

An alternate approach is based on the theory of mild solutions and is formulated in the context of evolutionary equations. In this approach, one proves the existence of a mild (also called integral) solution by using the fixed point theorem for strict contractions. This approach can be found in [9] (see also [39] or [28]). This method is in particular adapted to the proof of existence of classical solutions.

The uniqueness of strong solutions follows exactly the lines of the proof in the case of classical boundary conditions. 


\section{Auxiliary estimates}

We remind the reader of the convention described in the Introduction concerning the norms $\|\cdot\|_{L^{2}},\|\cdot\|_{H^{1}}$, etc; where $L^{2}=L^{2}\left(\Omega_{\varepsilon}\right)^{3}, H^{1}=H^{1}\left(\Omega_{\varepsilon}\right)^{3}$, etc. As indicated in the introduction, the vertical mean value of functions or vector fields plays an important role. We recall that the mean value operator $M$, defined in (1.4), acts on the scalar functions defined on $\Omega_{\varepsilon}$ and is given by

$$
M \varphi\left(x_{h}\right)=\frac{1}{\varepsilon g} \int_{0}^{\varepsilon g} \varphi\left(x_{h}, y_{3}\right) d y_{3},
$$

where $x_{h}=\left(x_{1}, x_{2}\right)$. We remark that, for $1 \leq p \leq \infty, M: L^{p}\left(\Omega_{\varepsilon}\right) \rightarrow L^{p}\left(\Omega_{\varepsilon}\right)$ is a bounded linear operator of norm 1 . We remark that $M$ is an orthogonal projection of $L^{2}\left(\Omega_{\varepsilon}\right)$ (for this property as well as for other properties, see [11], [12], [13]).

Next we introduce the notion of mean value for vectors. If $U=\left(U_{1}, U_{2}, U_{3}\right)$ belongs to $H_{\varepsilon}$, then $U$ is tangent to the boundary and, in particular, $U_{3}\left(x_{1}, x_{2}, 0\right)=0$, which implies by the Poincaré inequality that $\left\|U_{3}\right\|_{L^{2}} \leq \varepsilon\left\|U_{3}\right\|_{H^{1}}$. This indicates that taking the vertical mean value of the third component is not of real interest. Since the third component is small of order $\varepsilon$, we introduce the following mean value operator $M_{h}$ acting on $L^{1}\left(\Omega_{\varepsilon}\right)^{3}$ :

$$
M_{h} U\left(x_{h}\right)=\left(M U_{1}, M U_{2}, 0\right), \quad \text { for all } U \in L^{1}\left(\Omega_{\varepsilon}\right)^{3} .
$$

In order to keep the amount of new notation to the minimum, we also denote by $M_{h}$ the operator acting from $L^{1}\left(\Omega_{\varepsilon}\right)^{2}$ into $L^{1}\left(\Omega_{\varepsilon}\right)^{3}$, that is $M_{h}\left(Z_{1}, Z_{2}\right)=\left(M Z_{1}, M Z_{2}, 0\right)$ for any $Z=\left(Z_{1}, Z_{2}\right)$ in $L^{1}\left(\Omega_{\varepsilon}\right)^{2}$. We remark that, as above, $M_{h}: L^{p}\left(\Omega_{\varepsilon}\right)^{3} \rightarrow L^{p}\left(\Omega_{\varepsilon}\right)^{3}$ is a bounded linear operator of norm 1. Moreover, we point out that this operator $M_{h}$ is an orthogonal projection of $L^{2}\left(\Omega_{\varepsilon}\right)^{3}$.

Unfortunately the operator $M_{h}$ comes with a problem. In general, $M_{h} U$ does not belong to $H_{\varepsilon}$, when $U$ is in $H_{\varepsilon}$. For this reason, we introduce the following auxiliary mean value operator $M_{1}$ on $L^{1}\left(\Omega_{\varepsilon}\right)^{3}$ by

$$
M_{1} U(x)=\left(M U_{1}, M U_{2}, \frac{x_{3}}{g}\left(M_{h} U\right) \cdot \nabla g\right), \quad \text { for all } U \in L^{1}\left(\Omega_{\varepsilon}\right)^{3},
$$

(In Equation (3.2) and elsewhere, we denote by $\nabla g$ the usual gradient $\left(\partial_{1} g, \partial_{2} g, \partial_{3} g\right)=$ $\left(\partial_{1} g, \partial_{2} g, 0\right)$ ). One easily checks that, if $U$ belongs to $H_{\varepsilon}$, then $M_{1} U$ also belongs to $H_{\varepsilon}$ (in particular, $M_{1} U$ is divergence free and tangent to the boundary). We also point out that this projection is no longer orthogonal unless $g$ is a constant function. Like above, we also denote by $M_{1}$ the operator acting from $L^{1}\left(\Omega_{\varepsilon}\right)^{2}$ into $L^{1}\left(\Omega_{\varepsilon}\right)^{3}$, that is

$$
M_{1}\left(Z_{1}, Z_{2}\right)=\left(M Z_{1}, M Z_{2}, \frac{x_{3}}{g}\left(M_{h} Z\right) \cdot \nabla g\right), \quad \text { for any } Z=\left(Z_{1}, Z_{2}\right) \in L^{1}\left(\Omega_{\varepsilon}\right)^{2} .
$$

Remark 3.1. We already noticed that, if $\mathrm{U}$ belongs to $H_{\varepsilon}$, then the divergence of $M_{1} U$ vanishes too. We would also like to point out the following relation between the divergence of $M_{1} U$ and the divergence of $g M_{h} U$ :

$$
\operatorname{div}\left(M_{1} U\right)=\operatorname{div}\left(M_{h} U\right)+\frac{1}{g} \nabla g \cdot\left(M_{h} U\right)=\frac{1}{g} \operatorname{div}\left(g M_{h} U\right),
$$


which implies that

$$
\operatorname{div}\left(M_{1} U\right)=\frac{1}{g} \operatorname{div}\left(g M_{h} U\right)=0, \quad \text { for all } U \in H_{\varepsilon} .
$$

The quantity $\operatorname{div}(g \cdot)$ is sometimes called $g$-divergence. In Section 6 below, we will see that the limiting problem, when $\varepsilon$ goes to zero, deals only with two-dimensional vector fields $v$, such that the $g$-divergence of $(v, 0)$ vanishes. This $g$-divergence also appears in [19] dealing with the Euler equations in a thin domain like $\Omega_{\varepsilon}$.

Throughout this section, $u$ will denote a sufficiently smooth, divergence-free vector field that satisfies the Navier boundary conditions (1.3). As indicated above, we will decompose the vector field $u$ as follows

$$
u=v+w=M_{1} u+\left(I-M_{1}\right) u, \quad v_{h}=\left(v_{1}, v_{2}, 0\right)=\left(M u_{1}, M u_{2}, 0\right) .
$$

As we already noticed, $v$ and $w$ are divergence free, tangent to the boundary and periodic in $x_{1}$ and $x_{2}$. Moreover, $v$ and $w$ satisfy

$$
\left.\left.\left.\left.\left.\left.\left.\left.\left.\partial_{3} u_{1}\right|_{\Gamma_{0}} \equiv \partial_{3} v_{1}\right|_{\Gamma_{0}} \equiv \partial_{3} w_{1}\right|_{\Gamma_{0}} \equiv \partial_{3} u_{2}\right|_{\Gamma_{0}} \equiv \partial_{3} v_{2}\right|_{\Gamma_{0}} \equiv \partial_{3} w_{2}\right|_{\Gamma_{0}} \equiv u_{3}\right|_{\Gamma_{0}} \equiv v_{3}\right|_{\Gamma_{0}} \equiv w_{3}\right|_{\Gamma_{0}} \equiv 0
$$

that is $v$ and $w$ verify the Navier boundary conditions on $\Gamma_{0}$ but not necessarily on $\Gamma_{\varepsilon}$.

In our analysis below, it is convenient to use the following extension to $\Omega_{\varepsilon}$ of the exterior normal to $\partial \Omega_{\varepsilon}$ (see $\left.(2.1)\right)$ :

$$
N(x)=\left(\frac{-x_{3} \partial_{1} g}{g \sqrt{1+\varepsilon^{2}|\nabla g|^{2}}}, \frac{-x_{3} \partial_{2} g}{g \sqrt{1+\varepsilon^{2}|\nabla g|^{2}}}, \frac{x_{3}}{\varepsilon g}\left(\frac{1}{\sqrt{1+\varepsilon^{2}|\nabla g|^{2}}}+1\right)-1\right) .
$$

We will also use the function

$$
\widetilde{N}=\left(\frac{-x_{3} \partial_{1} g}{g \sqrt{1+\varepsilon^{2}|\nabla g|^{2}}}, \frac{-x_{3} \partial_{2} g}{g \sqrt{1+\varepsilon^{2}|\nabla g|^{2}}}, 1-\frac{\varepsilon x_{3}|\nabla g|^{2}}{g\left(1+\varepsilon^{2}|\nabla g|^{2}+\sqrt{1+\varepsilon^{2}|\nabla g|^{2}}\right)}\right)
$$

which is the exterior normal on $\Gamma_{\varepsilon}$ and interior normal on $\Gamma_{0}$. The advantage of $\widetilde{N}$ compared to $N$ is that the Lipschitz norm of $\widetilde{N}$ is bounded, independently of $\varepsilon$. More precisely, one can readily verify that there is a positive constant $C$, which does not depend on $\varepsilon$, such that the following two sets of inequalities hold, for all $\varepsilon$ with $0<\varepsilon \leq 1$,

$$
\begin{aligned}
& \|N\|_{L^{\infty}} \leq C, \quad\left\|\left(N_{1}, N_{2}\right)\right\|_{L^{\infty}} \leq C \varepsilon, \quad\|\nabla N\|_{L^{\infty}} \leq C \varepsilon^{-1} \\
& \left\|\nabla\left(N_{1}, N_{2}\right)\right\|_{L^{\infty}} \leq C, \quad\left\|\partial_{i} N\right\|_{L^{\infty}} \leq C, \quad\left\|\partial_{i} N_{j}\right\|_{L^{\infty}} \leq C \varepsilon \quad \text { for } i, j=1,2, \\
& \left\|\left(\widetilde{N}_{1}, \widetilde{N}_{2}\right)\right\|_{L^{\infty}} \leq C \varepsilon, \quad\left\|\widetilde{N}_{3}\right\|_{L^{\infty}} \leq C, \quad\left\|\nabla^{k} \tilde{N}\right\|_{L^{\infty}} \leq C \quad \text { for all } k \in \mathbb{N},
\end{aligned}
$$

and

$$
\left\|\partial_{i} \tilde{N}\right\|_{L^{\infty}} \leq C \varepsilon, \quad i=1,2
$$

We now recall the following consequence of the Stokes formula

$$
\int_{\partial \Omega_{\varepsilon}} \varphi(x) d \sigma_{x}=\int_{\Omega_{\varepsilon}} \varphi \operatorname{div} N d x+\int_{\Omega_{\varepsilon}} N \cdot \nabla \varphi d x
$$


where the boundary integral on $\Gamma_{\varepsilon}$ is defined by

$$
\int_{\Gamma_{\varepsilon}} \varphi(x) d \sigma_{x}=\int_{\mathbb{T}^{2}} \varphi\left(x_{h}, \varepsilon g\right) \sqrt{1+\varepsilon^{2}|\nabla g|^{2}} d x_{h}=\int_{\mathbb{T}^{2}} \frac{\varphi\left(x_{h}, \varepsilon g\right)}{N_{3}\left(x_{h}, \varepsilon g\right)} d x_{h} .
$$

The aim of this section is to prove several inequalities on $u$ and $w=\left(I-M_{1}\right) u$ with sharp dependence on $\varepsilon$ of the constants. First, we show how to estimate lower order Sobolev norms of $w$ in terms of higher order Sobolev norms of $w$ (see Proposition 3.6 below.) Next, we prove a regularity estimate connecting the $H^{2}$ norm of $u$ with the $L^{2}$ norm of $\Delta u$ (see Proposition 3.7). We continue with Proposition 3.9 which gives an estimate for the expression $\triangle u-\mathbb{P}_{\varepsilon} \triangle u$. We finally end this section with some $L^{p}$ estimates.

We start with a sequence of simple preliminary lemmas. The following lemma is wellknown (see [12], [13] for example). For the reader's convenience, we quickly recall its proof.

Lemma 3.1. Let $\varphi$ be a function in $H^{1}\left(\Omega_{\varepsilon}\right)$ satisfying either $\left.\varphi\right|_{\Gamma_{0}} \equiv 0$ or $M \varphi \equiv 0$. Then,

$$
\|\varphi\|_{L^{2}} \leq \varepsilon\|g\|_{L^{\infty}}\left\|\partial_{3} \varphi\right\|_{L^{2}}, \text { for all } \varepsilon>0 \text {. }
$$

Proof. In both cases, we remark that

$$
\iiint_{\substack{x_{h} \in \mathbb{T}^{2} \\ 0 \leq x_{3} \leq \varepsilon g\left(x_{h}\right)}} \varphi\left(x_{h}, x_{3}\right) \varphi\left(x_{h}, 0\right) d x_{h} d x_{3}=0,
$$

which implies that

$$
\begin{aligned}
\|\varphi\|_{L^{2}}^{2} & =\iiint_{\substack{x_{h} \in \mathbb{T}^{2} \\
0 \leq y_{3} \leq x_{3} \leq \varepsilon g\left(x_{h}\right)}} \varphi\left(x_{h}, x_{3}\right) \partial_{3} \varphi\left(x_{h}, y_{3}\right) d x_{h} d x_{3} d y_{3} \\
\leq & \left(\iiint_{\substack{x_{h} \in \mathbb{T}^{2} \\
0 \leq y_{3} \leq x_{3} \leq \varepsilon g\left(x_{h}\right)}} \varphi^{2}\left(x_{h}, x_{3}\right) d x_{h} d x_{3} d y_{3}\right)^{\frac{1}{2}}\left(\iiint_{\substack{x_{h} \in \mathbb{T}^{2} \\
0 \leq y_{3} \leq x_{3} \leq \varepsilon g\left(x_{h}\right)}}\left|\partial_{3} \varphi\left(x_{h}, y_{3}\right)\right|^{2} d x_{h} d x_{3} d y_{3}\right)^{\frac{1}{2}} \\
\leq & \left(\int_{\Omega_{\varepsilon}} \varphi^{2}(x) x_{3} d x\right)^{\frac{1}{2}}\left(\int_{\Omega_{\varepsilon}}\left|\partial_{3} \varphi(x)\right|^{2} \varepsilon g\left(x_{h}\right) d x\right)^{\frac{1}{2}} \\
\leq & \varepsilon\|g\|_{L^{\infty}}\|\varphi\|_{L^{2}}\left\|\partial_{3} \varphi\right\|_{L^{2}} .
\end{aligned}
$$

Lemma 3.2. Let $\varphi$ be a function in $H^{1}\left(\Omega_{\varepsilon}\right)$. Then,

$$
\|\varphi\|_{L^{2}} \leq\|M \varphi\|_{L^{2}}+\varepsilon\|g\|_{L^{\infty}}\left\|\partial_{3} \varphi\right\|_{L^{2}}, \quad \text { for all } \varepsilon \in(0,1] .
$$

Proof. The proof follows trivially from the previous lemma:

$$
\begin{aligned}
\|\varphi\|_{L^{2}} \leq\|M \varphi\|_{L^{2}}+\|(I-M) \varphi\|_{L^{2}} & \leq\|M \varphi\|_{L^{2}}+\varepsilon\|g\|_{L^{\infty}}\left\|\partial_{3}(I-M) \varphi\right\|_{L^{2}} \\
& =\|M \varphi\|_{L^{2}}+\varepsilon\|g\|_{L^{\infty}}\left\|\partial_{3} \varphi\right\|_{L^{2}}
\end{aligned}
$$


Lemma 3.3. For any $\varepsilon \in(0,1]$ and for any function $\varphi \in H^{1}\left(\Omega_{\varepsilon}\right)$, we have

$$
\left\|\varphi\left(x_{h}, \varepsilon g\left(x_{h}\right)\right)\right\|_{L^{2}} \leq 2\|M \varphi\|_{L^{2}}+\sqrt{5} \varepsilon\|g\|_{L^{\infty}}\left\|\partial_{3} \varphi\right\|_{L^{2}}, \quad \text { for all } \varepsilon \in(0,1] \text {. }
$$

Proof. We first remark that

$$
\left\|\varphi\left(x_{h}, \varepsilon g\left(x_{h}\right)\right)\right\|_{L^{2}}^{2}=\int_{\mathbb{T}^{2}} \varepsilon g \varphi^{2}\left(x_{h}, \varepsilon g\left(x_{h}\right)\right) d x_{h}=\int_{\mathbb{T}^{2}} \int_{0}^{\varepsilon g\left(x_{h}\right)} \frac{\partial}{\partial x_{3}}\left(x_{3} \varphi^{2}(x)\right) d x_{3} d x_{h} .
$$

Using Lemma 3.2 and the Young inequality, we infer from the previous equality that

$$
\begin{aligned}
\left\|\varphi\left(x_{h}, \varepsilon g\left(x_{h}\right)\right)\right\|_{L^{2}}^{2} & =\int_{\Omega_{\varepsilon}} \frac{\partial}{\partial x_{3}}\left(x_{3} \varphi^{2}(x)\right) d x=\|\varphi\|_{L^{2}}^{2}+2 \int_{\Omega_{\varepsilon}} x_{3} \varphi(x) \partial_{3} \varphi(x) d x \\
& \leq\|\varphi\|_{L^{2}}^{2}+2 \varepsilon\|g\|_{L^{\infty}}\|\varphi\|_{L^{2}}\left\|\partial_{3} \varphi\right\|_{L^{2}} \leq 2\|\varphi\|_{L^{2}}^{2}+\varepsilon^{2}\|g\|_{L^{\infty}}^{2}\left\|\partial_{3} \varphi\right\|_{L^{2}}^{2} \\
& \leq 4\|M \varphi\|_{L^{2}}^{2}+5 \varepsilon^{2}\|g\|_{L^{\infty}}^{2}\left\|\partial_{3} \varphi\right\|_{L^{2}}^{2}
\end{aligned}
$$

which implies the inequality of the lemma.

Lemma 3.4. There exists a positive constant $C$, which is independent of $\varepsilon$, such that, for all $\varepsilon \in(0,1]$, for any function $\varphi \in H^{2}\left(\Omega_{\varepsilon}\right)$, and for $i=1,2$, one has,

$$
\left\|\partial_{i} \varphi\right\|_{L^{2}} \leq C\|M \varphi\|_{H^{1}}+C \varepsilon\left\|\partial_{i} \partial_{3} \varphi\right\|_{L^{2}}+C \varepsilon\left\|\partial_{3} \varphi\right\|_{L^{2}}, \quad \text { for all } \varepsilon \in(0,1] \text {. }
$$

Proof. By Lemma 3.2, we obtain,

$$
\left\|\partial_{i} \varphi\right\|_{L^{2}} \leq\left\|M \partial_{i} \varphi\right\|_{L^{2}}+\varepsilon\|g\|_{L^{\infty}}\left\|\partial_{i} \partial_{3} \varphi\right\|_{L^{2}}, \quad \text { for } i=1,2 \text { and } \varepsilon \in(0,1] .
$$

On the other hand, a simple calculation shows that

$$
M \partial_{i} \varphi=\partial_{i} M \varphi+\frac{\partial_{i} g}{g}\left(M \varphi-\varphi\left(x_{h}, \varepsilon g\right)\right)
$$

so that, by Lemma 3.3,

$$
\left\|M \partial_{i} \varphi\right\|_{L^{2}} \leq C\|M \varphi\|_{H^{1}}+C\left\|\varphi\left(x_{h}, \varepsilon g\right)\right\|_{L^{2}} \leq C\|M \varphi\|_{H^{1}}+C \varepsilon\left\|\partial_{3} \varphi\right\|_{L^{2}},
$$

which completes the proof.

Lemma 3.5. There exists a positive constant $C$, which is independent of $\varepsilon$ such that, for all $\varepsilon \in(0,1]$ and for any function $\varphi \in H^{2}\left(\Omega_{\varepsilon}\right)$, one has

$$
\|M \varphi\|_{L^{2}} \leq\|\varphi\|_{L^{2}}, \quad\|M \varphi\|_{H^{1}} \leq C\|\varphi\|_{H^{1}}, \quad\|M \varphi\|_{H^{2}} \leq C\|\varphi\|_{H^{2}} .
$$

Proof. The first relation follows simply by noting that the operator $M$ is nothing else but the $L^{2}$ orthogonal projection onto the space of functions independent of the third variable. To prove the second inequality, we use the formula

$$
\partial_{i} M \varphi=M \partial_{i} \varphi-\frac{\partial_{i} g}{g}\left(M \varphi-\varphi\left(x_{h}, \varepsilon g\right)\right), \text { for } i=1,2,
$$

together with Lemma 3.3. Finally, the statement for the $H^{2}$ norm follows by differentiating the above relation and using similar arguments. We omit the details.

Remark 3.2. Lemma 3.5 directly implies that there exists a positive constant $C$, which is independent of $\varepsilon$ such that, for all $\varepsilon \in(0,1]$, for all $u \in H^{k}\left(\Omega_{\varepsilon}\right)^{3}, k=0,1,2$, the vectors $v=M_{1} u, v_{h}=M_{h} u$, satisfy,

$$
\|v\|_{H^{k}} \leq C\|u\|_{H^{k}}, \quad\left\|v_{h}\right\|_{H^{k}} \leq C\|u\|_{H^{k}}, \text { for } k=0,1,2 \text {. }
$$


We can now prove the following proposition.

Proposition 3.6. There exists a positive constant $C$, which is independent of $\varepsilon$, such that, for all $\varepsilon \in(0,1]$ and for all $u$ that belongs to $V_{\varepsilon}$, the functions $w=\left(I-M_{1}\right) u$ and $w^{*}=\left(I-M_{h}\right) u$ satisfy,

$$
\begin{aligned}
\|w\|_{L^{2}} & \leq C \varepsilon\left\|\partial_{3} w\right\|_{L^{2}}, \quad \text { for all } \varepsilon \in(0,1] \\
\left\|w^{*}\right\|_{L^{2}} & \leq C \varepsilon\left\|\partial_{3} w^{*}\right\|_{L^{2}}, \quad \text { for all } \varepsilon \in(0,1],
\end{aligned}
$$

and, if moreover $u$ belongs to $H^{2}\left(\Omega_{\varepsilon}\right)^{3}$ and satisfies the Navier boundary conditions (1.3),

$$
\|\nabla w\|_{L^{2}} \leq C \varepsilon\left\|\nabla^{2} w\right\|_{L^{2}}, \quad \text { for all } \varepsilon \in(0,1] .
$$

Proof. Relation (3.13) follows immediately from Lemma 3.1 since $M w_{1} \equiv M w_{2} \equiv 0$ and $\left.w_{3}\right|_{\Gamma_{0}}=\left.w_{3}^{*}\right|_{\Gamma_{0}} \equiv 0$. To prove (3.14) we take different cases. First, for $i \in\{1,2\}$, we have that $\partial_{3} w_{i} \equiv \partial_{i} w_{3} \equiv 0$ on $\Gamma_{0}$ so, by Lemma 3.1,

$$
\left\|\partial_{3} w_{i}\right\|_{L^{2}} \leq \varepsilon\|g\|_{L^{\infty}}\left\|\partial_{3}^{2} w_{i}\right\|_{L^{2}} \quad \text { and } \quad\left\|\partial_{i} w_{3}\right\|_{L^{2}} \leq \varepsilon\|g\|_{L^{\infty}}\left\|\partial_{3} \partial_{i} w_{3}\right\|_{L^{2}} .
$$

Next, for $i, j \in\{1,2\}$ we use that $M w_{i} \equiv 0$ and Lemma 3.4 to deduce that

$$
\left\|\partial_{j} w_{i}\right\|_{L^{2}} \leq C \varepsilon\left\|\partial_{j} \partial_{3} w_{i}\right\|_{L^{2}}+C \varepsilon\left\|\partial_{3} w_{i}\right\|_{L^{2}} \leq C \varepsilon\left\|\partial_{j} \partial_{3} w_{i}\right\|_{L^{2}}+C \varepsilon^{2}\left\|\partial_{3}^{2} w_{i}\right\|_{L^{2}} \leq C \varepsilon\left\|\nabla^{2} w\right\|_{L^{2}} .
$$

Finally, the estimate for $\partial_{3} w_{3}$ follows at once from the above bounds together with the divergence free condition

$$
\left\|\partial_{3} w_{3}\right\|_{L^{2}}=\left\|\partial_{1} w_{1}+\partial_{2} w_{2}\right\|_{L^{2}} \leq C \varepsilon\left\|\nabla^{2} w\right\|_{L^{2}} .
$$

This completes the proof.

The next result is a regularity type estimate for $u$.

Proposition 3.7. There exist a real number $\varepsilon_{0}$, with $0<\varepsilon_{0} \leq 1$ and a positive constant $C$, which is independent of $\varepsilon$, such that, for all $\varepsilon \in\left(0, \varepsilon_{0}\right)$, for all $u \in V_{\varepsilon} \cap H^{2}\left(\Omega_{\varepsilon}\right)^{3}$ satisfying the Navier boundary conditions (1.3), one has

$$
\|u\|_{H^{2}} \leq C\|\triangle u\|_{L^{2}}+C\|u\|_{H^{1}}, \quad \text { for all } \varepsilon \in\left(0, \varepsilon_{0}\right] .
$$

Proof. Assume for the moment that $\left\|\nabla^{2} u\right\|_{L^{2}}^{2}$ satisfies

$$
\left\|\nabla^{2} u\right\|_{L^{2}}^{2} \leq\|\Delta u\|_{L^{2}}^{2}+C_{0} \varepsilon\|u\|_{H^{2}}^{2}+C\|u\|_{H^{2}}\|u\|_{H^{1}},
$$

where $C_{0}$ and $C$ are positive constants which do not depend on $\varepsilon$. One then finds that

$$
\begin{aligned}
\|u\|_{H^{2}}^{2} & \leq\|\Delta u\|_{L^{2}}^{2}+C_{0} \varepsilon\|u\|_{H^{2}}^{2}+C\|u\|_{H^{2}}\|u\|_{H^{1}}+\|u\|_{H^{1}}^{2} \\
& \leq\|\Delta u\|_{L^{2}}^{2}+C_{0} \varepsilon\|u\|_{H^{2}}^{2}+\frac{1}{4}\|u\|_{H^{2}}^{2}+C\|u\|_{H^{1}}^{2} .
\end{aligned}
$$

Now choose $\varepsilon_{0}$ to satisfy $0<\varepsilon_{0} \leq 1$ and $C_{0} \varepsilon_{0} \leq 1 / 4$. One then obtains, for $0<\varepsilon \leq \varepsilon_{0}$,

$$
\frac{1}{2}\|u\|_{H^{2}}^{2} \leq\|\triangle u\|_{L^{2}}^{2}+C\|u\|_{H^{1}}^{2}
$$

which implies the proposition. 
It remains to estimate the term $\left\|\nabla^{2} u\right\|_{L^{2}}$. We write

$$
\begin{aligned}
\left\|\nabla^{2} u\right\|_{L^{2}}^{2} & =\sum_{i, j=1}^{3} \int_{\Omega_{\varepsilon}} \partial_{i} \partial_{j} u \cdot \partial_{i} \partial_{j} u d x \\
& =-\sum_{i, j=1}^{3} \int_{\Omega_{\varepsilon}} \partial_{j} u \cdot \partial_{i}^{2} \partial_{j} u d x+\sum_{i, j=1}^{3} \int_{\partial \Omega_{\varepsilon}} N_{i} \partial_{j} u \cdot \partial_{i} \partial_{j} u d \sigma_{x} \\
& =\sum_{i, j=1}^{3} \int_{\Omega_{\varepsilon}} \partial_{j}^{2} u \cdot \partial_{i}^{2} u d x-\sum_{i, j=1}^{3} \int_{\partial \Omega_{\varepsilon}} N_{j} \partial_{j} u \cdot \partial_{i}^{2} u d \sigma_{x}+\sum_{i, j=1}^{3} \int_{\partial \Omega_{\varepsilon}} N_{i} \partial_{j} u \cdot \partial_{i} \partial_{j} u d \sigma_{x} \\
& =\int_{\Omega_{\varepsilon}}|\triangle u|^{2} d x+\sum_{i, j=1}^{3} \int_{\partial \Omega_{\varepsilon}} \partial_{j} u \cdot\left(N_{i} \partial_{j}-N_{j} \partial_{i}\right) \partial_{i} u d \sigma_{x}
\end{aligned}
$$

where we integrated twice by parts. It therefore suffices to bound the last term that we decompose as follows

$$
\begin{aligned}
\sum_{i, j=1}^{3} \int_{\partial \Omega_{\varepsilon}} \partial_{j} u \cdot\left(N_{i} \partial_{j}-N_{j} \partial_{i}\right) \partial_{i} u d \sigma_{x}= & 2 \underbrace{\int_{\partial \Omega_{\varepsilon}} \partial_{2} u \cdot\left(N_{1} \partial_{2}-N_{2} \partial_{1}\right) \partial_{1} u d \sigma_{x}}_{I_{1}} \\
& +2 \sum_{i=1}^{2} \underbrace{\int_{\partial \Omega_{\varepsilon}} \partial_{3} u_{3}\left(N_{i} \partial_{3}-N_{3} \partial_{i}\right) \partial_{i} u_{3} d \sigma_{x}}_{I_{2}} \\
& +2 \sum_{i, k=1}^{2} \underbrace{\int_{\partial \Omega_{\varepsilon}} \partial_{3} u_{k}\left(N_{i} \partial_{3}-N_{3} \partial_{i}\right) \partial_{i} u_{k} d \sigma_{x}}_{I_{3}}
\end{aligned}
$$

We used above that the expression $\int_{\partial \Omega_{\varepsilon}} \partial_{j} u \cdot\left(N_{i} \partial_{j}-N_{j} \partial_{i}\right) \partial_{i} u d \sigma_{x}$ is symmetric in $i$ and $j$, for $i \neq j$; this in turn follows after noticing that $N_{i} \partial_{j}-N_{j} \partial_{i}$ is tangent to the boundary and performing an integration by parts on the boundary. We now estimate separately the three terms $I_{1}, I_{2}$ and $I_{3}$.

Estimate of $I_{1}$. We return to an integral on $\Omega_{\varepsilon}$ by using formula (3.10)

$$
\begin{aligned}
I_{1}= & \int_{\Omega_{\varepsilon}} \partial_{2} u \cdot\left(N_{1} \partial_{2}-N_{2} \partial_{1}\right) \partial_{1} u \operatorname{div} N d x+\int_{\Omega_{\varepsilon}} N \cdot \nabla\left[\partial_{2} u \cdot\left(N_{1} \partial_{2}-N_{2} \partial_{1}\right) \partial_{1} u\right] d x \\
= & \underbrace{\int_{\Omega_{\varepsilon}} \partial_{2} u \cdot\left(N_{1} \partial_{2}-N_{2} \partial_{1}\right) \partial_{1} u \operatorname{div} N d x}_{I_{11}}+\sum_{k=1}^{3}[\underbrace{\int_{\Omega_{\varepsilon}} N_{k} \partial_{k} \partial_{2} u \cdot\left(N_{1} \partial_{2}-N_{2} \partial_{1}\right) \partial_{1} u d x}_{I_{12}} \\
& +\underbrace{\int_{\Omega_{\varepsilon}} N_{k} \partial_{2} u \cdot\left(\partial_{k} N_{1} \partial_{2}-\partial_{k} N_{2} \partial_{1}\right) \partial_{1} u d x}_{I_{13}}+\underbrace{\int_{\Omega_{\varepsilon}} N_{k} \partial_{2} u \cdot\left(N_{1} \partial_{2}-N_{2} \partial_{1}\right) \partial_{k} \partial_{1} u d x}_{I_{14}}]
\end{aligned}
$$


Using relations (3.7) we readily estimate

$$
\left|I_{11}\right|+\left|I_{13}\right| \leq C\|u\|_{H^{1}}\left\|\nabla^{2} u\right\|_{L^{2}} \text { and }\left|I_{12}\right| \leq C \varepsilon\left\|\nabla^{2} u\right\|_{L^{2}}^{2}
$$

Regarding $I_{14}$ we need to make another integration by parts of the derivatives $\partial_{1}, \partial_{2}$ from $N_{1} \partial_{2}-N_{2} \partial_{1}$ and deduce as above that

$$
\begin{aligned}
\left|I_{14}\right| & =\left|\int_{\Omega_{\varepsilon}}\left(N_{2} \partial_{1}-N_{1} \partial_{2}\right)\left(N_{k} \partial_{2} u\right) \cdot \partial_{k} \partial_{1} u d x+\int_{\Omega_{\varepsilon}}\left(\partial_{1} N_{2}-\partial_{2} N_{1}\right) N_{k} \partial_{2} u \cdot \partial_{k} \partial_{1} u d x\right| \\
& \leq C \varepsilon\|u\|_{H^{1}}\left\|\nabla^{2} u\right\|_{L^{2}}+C \varepsilon\left\|\nabla^{2} u\right\|_{L^{2}}^{2}
\end{aligned}
$$

Therefore we obtain,

$$
\left|I_{1}\right| \leq C\|u\|_{H^{1}}\left\|\nabla^{2} u\right\|_{L^{2}}+C \varepsilon\left\|\nabla^{2} u\right\|_{L^{2}}^{2}
$$

Estimate of $I_{2}$. To estimate $I_{2}$, we argue as in the proof of Theorem 2.2 by using the fact that $u$ is tangent to $\Gamma_{\varepsilon}$, i.e.

$$
u_{3}\left(x_{h}, \varepsilon g\right)=\varepsilon u_{h}\left(x_{h}, \varepsilon g\right) \cdot \nabla g \quad \text { for all } x_{h} \in \mathbb{T}^{2},
$$

where $u_{h}=\left(u_{1}, u_{2}, 0\right)$ denotes the horizontal part of $u$. For $i \in\{1,2\}$ we differentiate this equality with respect to $x_{i}$ to deduce that

$$
\begin{aligned}
\partial_{i} u_{3}\left(x_{h}, \varepsilon g\right)= & \varepsilon u_{h}\left(x_{h}, \varepsilon g\right) \cdot \nabla \partial_{i} g+\varepsilon \partial_{i} u_{h}\left(x_{h}, \varepsilon g\right) \cdot \nabla g \\
& +\varepsilon^{2} \partial_{i} g \partial_{3} u_{h}\left(x_{h}, \varepsilon g\right) \cdot \nabla g-\varepsilon \partial_{i} g \partial_{3} u_{3}\left(x_{h}, \varepsilon g\right)=\varepsilon g F_{\varepsilon}^{i}\left(u\left(x_{h}, \varepsilon g\right), g\right)
\end{aligned}
$$

where the function

$$
F_{\varepsilon}^{i}(u, g) \equiv\left[u_{h} \cdot \nabla \partial_{i} g+\partial_{i} u_{h} \cdot \nabla g+\varepsilon \partial_{i} g \partial_{3} u_{h} \cdot \nabla g-\partial_{i} g \partial_{3} u_{3}\right] g^{-1}
$$

is defined on $\bar{\Omega}_{\varepsilon}$. We just proved that $\partial_{i} u_{3}=x_{3} F_{\varepsilon}^{i}(u, g)$ on $\Gamma_{\varepsilon}$. Moreover, according to (3.5), this equality also holds on $\Gamma_{0}$, since $\partial_{i} u_{3}$ vanishes on $\Gamma_{0}$ for $i=1,2$. Thus,

$$
I_{2}=\int_{\partial \Omega_{\varepsilon}} \partial_{3} u_{3}\left(N_{i} \partial_{3}-N_{3} \partial_{i}\right)\left(x_{3} F_{\varepsilon}^{i}(u, g)\right) d \sigma_{x}
$$

Performing an integration by parts, we go back to an integral on $\Omega_{\varepsilon}$ as follows,

$$
\begin{aligned}
I_{2} & =\int_{\Omega_{\varepsilon}}\left\{\partial_{i}\left[\partial_{3} u_{3} \partial_{3}\left(x_{3} F_{\varepsilon}^{i}(u, g)\right)\right]-\partial_{3}\left[\partial_{3} u_{3} \partial_{i}\left(x_{3} F_{\varepsilon}^{i}(u, g)\right)\right]\right\} d x \\
& =\int_{\Omega_{\varepsilon}}\left[\partial_{i} \partial_{3} u_{3} F_{\varepsilon}^{i}(u, g)+\partial_{i} \partial_{3} u_{3} x_{3} \partial_{3} F_{\varepsilon}^{i}(u, g)-\partial_{3}^{2} u_{3} x_{3} \partial_{i} F_{\varepsilon}^{i}(u, g)\right] d x .
\end{aligned}
$$

It now suffices to note that $x_{3}=O(\varepsilon)$ and to take into account the expression for $F_{\varepsilon}^{i}(u, g)$ to get,

$$
\left|I_{2}\right| \leq C\|u\|_{H^{1}}\left\|\nabla^{2} u\right\|_{L^{2}}+C \varepsilon\|u\|_{H^{2}}^{2} .
$$


Estimate of $I_{3}$. It suffices to estimate the term $I_{31 i}=\int_{\partial \Omega_{\varepsilon}} \partial_{3} u_{1}\left(N_{i} \partial_{3}-N_{3} \partial_{i}\right) \partial_{i} u_{1} d \sigma_{x}$ corresponding to $k=1$. Since $D(u) N$ is normal to the boundary $\Gamma_{\varepsilon}$ and $\left(1,0, \varepsilon \partial_{1} g\right)$ is tangent to this portion of boundary, we deduce that

$$
D(u) N \cdot\left(1,0, \varepsilon \partial_{1} g\right)=0, \quad \text { on } \quad \Gamma_{\varepsilon}
$$

This can be expanded as

$$
\begin{aligned}
-2 \varepsilon \partial_{1} g \partial_{1} u_{1} & -\varepsilon \partial_{2} g\left(\partial_{1} u_{2}+\partial_{2} u_{1}\right)+\partial_{1} u_{3}+\partial_{3} u_{1} \\
& +\varepsilon \partial_{1} g\left[-\varepsilon \partial_{1} g\left(\partial_{1} u_{3}+\partial_{3} u_{1}\right)-\varepsilon \partial_{2} g\left(\partial_{2} u_{3}+\partial_{3} u_{2}\right)+2 \partial_{3} u_{3}\right]=0 \quad \text { on } \Gamma_{\varepsilon} .
\end{aligned}
$$

We now use the above relation and (3.17) for $i=1$ to deduce that

$$
\partial_{3} u_{1}=x_{3} H_{\varepsilon}(u, g) \quad \text { on } \quad \Gamma_{\varepsilon},
$$

where

$$
\begin{aligned}
H_{\varepsilon}(u, g)=-F_{\varepsilon}^{1}(u, g)+\{ & 2 \partial_{1} g \partial_{1} u_{1}+\partial_{2} g\left(\partial_{1} u_{2}+\partial_{2} u_{1}\right) \\
& \left.\quad+\partial_{1} g\left[\varepsilon \partial_{1} g\left(\partial_{1} u_{3}+\partial_{3} u_{1}\right)+\varepsilon \partial_{2} g\left(\partial_{2} u_{3}+\partial_{3} u_{2}\right)-2 \partial_{3} u_{3}\right]\right\} g^{-1}
\end{aligned}
$$

It is clear from this formula that

$$
\left\|H_{\varepsilon}(u, g)\right\|_{L^{2}} \leq C\|u\|_{H^{1}} \quad \text { and } \quad\left\|\nabla H_{\varepsilon}(u, g)\right\|_{L^{2}} \leq C\|u\|_{H^{2}},
$$

for some constant $C$, which is independent of $\varepsilon$. We now proceed as in the estimate for $I_{2}$ by returning to an integral on $\Omega_{\varepsilon}$. One has that

$$
\begin{aligned}
I_{31 i} & =\int_{\partial \Omega_{\varepsilon}} \partial_{3} u_{1}\left(N_{i} \partial_{3}-N_{3} \partial_{i}\right) \partial_{i} u_{1} d \sigma_{x} \\
& =\int_{\partial \Omega_{\varepsilon}} x_{3} H_{\varepsilon}\left(N_{i} \partial_{3}-N_{3} \partial_{i}\right) \partial_{i} u_{1} d \sigma_{x} \\
& =\int_{\Omega_{\varepsilon}}\left[\partial_{i}\left(x_{3} H_{\varepsilon} \partial_{3} \partial_{i} u_{1}\right)-\partial_{3}\left(x_{3} H_{\varepsilon} \partial_{i}^{2} u_{1}\right)\right] d x \\
& =\int_{\Omega_{\varepsilon}}\left[x_{3} \partial_{i} H_{\varepsilon} \partial_{3} \partial_{i} u_{1}-x_{3} \partial_{3} H_{\varepsilon} \partial_{i}^{2} u_{1}-H_{\varepsilon} \partial_{i}^{2} u_{1}\right] d x
\end{aligned}
$$

Since $x_{3}=O(\varepsilon)$, we use $(3.20)$ to obtain

$$
I_{31 i} \leq C\|u\|_{H^{2}}\left(\varepsilon\|u\|_{H^{2}}+\|u\|_{H^{1}}\right) .
$$

The inequality (3.15) now follows from (3.16), (3.18) and (3.21).

Let us now observe that the Poincaré inequality holds true with constants independent of $\varepsilon$.

Lemma 3.8. There exists a positive constant $C$, which is independent of $\varepsilon$, such that for every $\varphi \in H^{1}\left(\Omega_{\varepsilon}\right)$ with $\int_{\Omega_{\varepsilon}} \varphi d x=0$, one has

$$
\|\varphi\|_{L^{2}} \leq C\|\nabla \varphi\|_{L^{2}}, \quad \text { for all } \varepsilon \in(0,1] .
$$


Proof. The proof is simply a matter of rescaling. Let $\varphi_{\varepsilon}(x)=\varphi\left(x_{h}, \varepsilon x_{3}\right)$. Then $\varphi_{\varepsilon}$ is a function defined on a domain independent of $\varepsilon$ and has vanishing mean. The standard Poincaré inequality therefore gives

$$
\left\|\varphi_{\varepsilon}\right\|_{L^{2}} \leq C\left\|\nabla \varphi_{\varepsilon}\right\|_{L^{2}}
$$

for some constant $C$ independent of $\varepsilon$. Expressing this relation in terms of $\varphi$ we obtain

$$
\varepsilon^{-\frac{1}{2}}\|\varphi\|_{L^{2}} \leq C \varepsilon^{-\frac{1}{2}}\left(\left\|\partial_{1} \varphi\right\|_{L^{2}}^{2}+\left\|\partial_{2} \varphi\right\|_{L^{2}}^{2}+\varepsilon^{2}\left\|\partial_{3} \varphi\right\|_{L^{2}}^{2}\right)^{\frac{1}{2}}
$$

The conclusion follows.

We observe next that $\triangle u$ is tangent to $\Gamma_{0}$, the flat part of the boundary. If this would be true on $\Gamma_{\varepsilon}$ too, then we would get that $\mathbb{P}_{\varepsilon} \Delta u=\Delta u$. Since this is not exactly true we can prove only the following statement:

Proposition 3.9. There exists a positive constant $C$, which is independent of $\varepsilon$, such that, for all $\varepsilon \in(0,1)$, for any vector $u \in H_{\varepsilon} \cap H^{2}\left(\Omega_{\varepsilon}\right)^{3}$ that satisfies the Navier boundary conditions (1.3), one has

$$
\left\|\triangle u-\mathbb{P}_{\varepsilon} \triangle u\right\|_{L^{2}} \leq C\|u\|_{H^{1}}+C \varepsilon\|u\|_{H^{2}}, \quad \text { for all } \varepsilon \in(0,1] .
$$

Proof. By the properties of the projection $\mathbb{P}_{\varepsilon}$, we know that there is some scalar function $q$ such that

$$
\triangle u-\mathbb{P}_{\varepsilon} \triangle u=\nabla q .
$$

We can assume without loss of generality that $q$ has vanishing mean on $\Omega_{\varepsilon}$. Taking the divergence of this relation and also the scalar product with the exterior normal restricted to the boundary we see that $q$ verifies the following Neumann problem

$$
\triangle q=0,\left.\left.\quad N \cdot \nabla q\right|_{\partial \Omega_{\varepsilon}} \equiv N \cdot \triangle u\right|_{\partial \Omega_{\varepsilon}} .
$$

Now, it so happens that $\left.N \cdot \triangle u\right|_{\partial \Omega_{\varepsilon}}$ can be expressed in terms of lower order derivatives of $u$. More precisely, the following identity is proved in the course of the proof of [2, Proposition 2]:

$$
\begin{aligned}
-N \cdot \Delta u= & N \cdot\left[(N \times \nabla) \times\left(2 N \times \sum_{i} u_{i}(N \times \nabla) N_{i}\right)\right] \\
& -N \cdot[(N \times \nabla) \times(N \times \operatorname{curl} u)-N(N \cdot(\nabla \times \operatorname{curl} u))] \text { on the boundary, }
\end{aligned}
$$

which holds for any smooth extension $N$ of the exterior normal inside $\Omega_{\varepsilon}$ and any vector field $u$ verifying the Navier boundary conditions. We further note that the following identity holds true

$$
(N \times \nabla) \times(N \times \operatorname{curl} u)-N(N \cdot(\nabla \times \operatorname{curl} u))=\left(\operatorname{curl} u \cdot \nabla_{\tau}\right) N-\operatorname{curl} u\left(\nabla_{\tau} \cdot N\right),
$$

where we denote by $\nabla_{\tau}$ the vector of tangential derivatives defined by

$$
\nabla_{\tau}=N \times \nabla=\left(\begin{array}{l}
N_{2} \partial_{3}-N_{3} \partial_{2} \\
N_{3} \partial_{1}-N_{1} \partial_{3} \\
N_{1} \partial_{2}-N_{2} \partial_{1}
\end{array}\right)
$$


We deduce that the following identity holds on the boundary:

$$
-N \cdot \triangle u=N \cdot\left[\nabla_{\tau} \times\left(2 N \times \sum_{i} u_{i} \nabla_{\tau} N_{i}\right)\right]-N \cdot\left[\left(\operatorname{curl} u \cdot \nabla_{\tau}\right) N-\operatorname{curl} u\left(\nabla_{\tau} \cdot N\right)\right] .
$$

Let us introduce

$$
G(u, \widetilde{N})=\left(\operatorname{curl} u \cdot \nabla_{\widetilde{\tau}}\right) \widetilde{N}-\operatorname{curl} u\left(\nabla_{\widetilde{\tau}} \cdot \widetilde{N}\right)-\nabla_{\widetilde{\tau}} \times\left(2 \widetilde{N} \times \sum_{i} u_{i} \nabla_{\widetilde{\tau}} \widetilde{N}_{i}\right),
$$

where

$$
\nabla_{\widetilde{\tau}}=\tilde{N} \times \nabla=\left(\begin{array}{c}
\widetilde{N}_{2} \partial_{3}-\widetilde{N}_{3} \partial_{2} \\
\widetilde{N}_{3} \partial_{1}-\widetilde{N}_{1} \partial_{3} \\
\widetilde{N}_{1} \partial_{2}-\widetilde{N}_{2} \partial_{1}
\end{array}\right)
$$

Since $N \equiv \widetilde{N}$ on $\Gamma_{\varepsilon}$ and $N \equiv-\widetilde{N}$ on $\Gamma_{0}$ we deduce that

$$
N \cdot \triangle u=N \cdot G(u, \tilde{N}) \quad \text { on } \partial \Omega_{\varepsilon} .
$$

We can now go to the estimate of $\nabla q$. We integrate by parts, use the Stokes formula together with relations (3.23) and (3.25) to deduce that

$$
\begin{aligned}
\|\nabla q\|_{L^{2}}^{2}=-\int_{\Omega_{\varepsilon}} q \triangle q d x+\int_{\partial \Omega_{\varepsilon}} q N \cdot \nabla q d \sigma_{x}=\int_{\partial \Omega_{\varepsilon}} q N \cdot G(u, \tilde{N}) d \sigma_{x} \\
=\int_{\Omega_{\varepsilon}} \operatorname{div}(q G(u, \tilde{N})) d x=\underbrace{\int_{\Omega_{\varepsilon}} G(u, \tilde{N}) \cdot \nabla q d x}_{J_{1}}+\underbrace{\int_{\Omega_{\varepsilon}} q \operatorname{div}(G(u, \tilde{N})) d x}_{J_{2}}
\end{aligned}
$$

To estimate $J_{1}$ we simply use the Hölder inequality together with relations (3.8) and the explicit formula for $G$ :

$$
J_{1} \leq\|\nabla q\|_{L^{2}}\|G(u, \tilde{N})\|_{L^{2}} \leq C\|\nabla q\|_{L^{2}}\|u\|_{H^{1}}
$$

for some constant $C$ independent of $\varepsilon$.

The estimate for $J_{2}$ is more delicate as this term involves second order derivatives of $u$. The point is that in $G$ we can find tangential derivatives from $\nabla_{\widetilde{\tau}}$ applied to components of $\widetilde{N}$. Looking carefully at the expression $(3.24)$ of $\nabla_{\widetilde{\tau}}$ and at the equality defining $G$, we see that the second order derivatives of $u$ are either multiplied by terms of the form $\widetilde{N}_{i} \partial_{j} \widetilde{N}, i=1,2, j=1,2,3$, or by terms of the form $\widetilde{N}_{3} \partial_{i} \widetilde{N}, i=1,2$. Due to the estimates (3.8) and (3.9), all these factors are of order $\varepsilon$. By the explicit formula for $\widetilde{N}$ given in (3.6) we see that these tangential derivatives have uniform norm bounded like $O(\varepsilon)$. However, we should pay attention to the fact the derivatives of these tangential derivatives are not bounded better than $O(1)$. In conclusion, the following analysis can be done in order to estimate the $L^{2}$ norm of $\operatorname{div}(G(u, \widetilde{N}))$. When expanding the expression for $\operatorname{div}(G(u, \widetilde{N}))$ two type of terms show up:

- either all derivatives go on $u$ which means that no derivative is applied to the tangential derivatives of $\widetilde{N}$; we infer that the $L^{2}$ norm of these terms is bounded by $C \varepsilon\|u\|_{H^{2}}$.

- or at most one derivative goes on $u$; in view of (3.8) these terms are bounded by $C\|u\|_{H^{1}}$. 
From this discussion it follows that $J_{2}$ can be bounded by

$$
\begin{aligned}
J_{2} \leq\|q\|_{L^{2}}\|\operatorname{div}(G(u, \tilde{N}))\|_{L^{2}} & \leq C\|q\|_{L^{2}}\left(\varepsilon\|u\|_{H^{2}}+\|u\|_{H^{1}}\right) \\
& \leq C\|\nabla q\|_{L^{2}}\left(\varepsilon\|u\|_{H^{2}}+\|u\|_{H^{1}}\right),
\end{aligned}
$$

where we used the Poincaré inequality of Lemma 3.8.

Putting together the estimates we have for $J_{1}$ and $J_{2}$, we infer that

$$
\|\nabla q\|_{L^{2}}^{2} \leq C\|\nabla q\|_{L^{2}}\left(\varepsilon\|u\|_{H^{2}}+\|u\|_{H^{1}}\right), \quad \text { for all } \varepsilon \in\left(0, \varepsilon_{0}\right] .
$$

In view of (3.22) this completes the proof.

We next recall two auxiliary inequalities, which are very useful in estimating the quadratic form.

Lemma 3.10. There exist positive constants $\varepsilon_{0}$ and $C$ (independent of $\varepsilon$ ) such that, for any $0<\varepsilon \leq \varepsilon_{0}$, the following inequalities hold. For any $\varphi \in H^{1}\left(\Omega_{\varepsilon}\right)$ such that either $\left.\varphi\right|_{\Gamma_{0}} \equiv 0$ or $M \varphi \equiv 0$,

$$
\|\varphi\|_{L^{q}} \leq C \varepsilon^{\frac{3}{q}-\frac{1}{2}}\|\nabla \varphi\|_{L^{2}}, \quad \text { for all } q \text { with } 2 \leq q \leq 6 .
$$

In particular, for $w=\left(I-M_{1}\right) u$ and $w^{*}=\left(I-M_{h}\right) u$, where $u \in V_{\varepsilon}$, one has,

$$
\begin{aligned}
\|w\|_{L^{q}} & \leq C \varepsilon^{\frac{3}{q}-\frac{1}{2}}\|\nabla w\|_{L^{2}}, \\
\left\|w^{*}\right\|_{L^{q}} & \leq C \varepsilon^{\frac{3}{q}-\frac{1}{2}}\left\|\nabla w^{*}\right\|_{L^{2}}, \quad \text { for all q with } 2 \leq q \leq 6 .
\end{aligned}
$$

Moreover, if $u \in H^{2}\left(\Omega_{\varepsilon}\right)^{3} \cap V_{\varepsilon}$ satisfies the Navier boundary conditions, we have,

$$
\|\nabla w\|_{L^{q}} \leq C \varepsilon^{\frac{3}{q}-\frac{1}{2}}\|w\|_{H^{2}}, \quad \text { for all } 2 \leq q \leq 6 .
$$

Proof. According to Proposition 4.2 and Lemma 6.1 of [12], using the classical Sobolev inequalities, we can write

$$
\|\varphi\|_{L^{6}} \leq C\|\nabla \varphi\|_{L^{2}}
$$

where the constant $C$ is independent of $\varepsilon$. By interpolation between $L^{2}\left(\Omega_{\varepsilon}\right)$ and $L^{6}\left(\Omega_{\varepsilon}\right)$, we deduce the inequality (3.26) from the inequality (3.29) and from Lemma 3.1. The estimate (3.27) is an obvious consequence of (3.26).

It remains to prove the estimate (3.28). We simply write, for $i, j \in\{1,2,3\}$,

$$
\left\|\partial_{i} w_{j}\right\|_{L^{q}} \leq C\left(\left\|M \partial_{i} w_{j}\right\|_{L^{q}}+\left\|(I-M) \partial_{i} w_{j}\right\|_{L^{q}}\right) \leq C\left(\left\|M \partial_{i} w_{j}\right\|_{L^{q}}+\varepsilon^{\frac{3}{q}-\frac{1}{2}}\|w\|_{H^{2}}\right)
$$

By the two-dimensional Gagliardo-Nirenberg inequality (see [27]) and (3.14), we obtain,

$$
\begin{aligned}
\left\|M \partial_{i} w_{j}\right\|_{L^{q}} & \leq C \varepsilon^{\frac{1}{q}}\left\|M \partial_{i} w_{j}\right\|_{L^{2}\left(\mathbb{T}^{2}\right)}^{2 / q}\left\|M \partial_{i} w_{j}\right\|_{H^{1}\left(\mathbb{T}^{2}\right)}^{1-2 / q} \\
& \leq C \varepsilon^{\frac{1}{q}-\frac{1}{2}}\left\|M \partial_{i} w_{j}\right\|_{L^{2}}^{2 / q}\left\|M \partial_{i} w_{j}\right\|_{H^{1}}^{1-2 / q} \\
& \leq C \varepsilon^{\frac{3}{q}-\frac{1}{2}}\|w\|_{H^{2}} .
\end{aligned}
$$

The inequalities (3.30) and (3.31) imply the inequality (3.28). 
Lemma 3.11. There exist positive constants $\varepsilon_{0}$ and $C$, which are independent of $\varepsilon$, such that, for any $0<\varepsilon \leq \varepsilon_{0}$, the following estimate holds for $w=\left(I-M_{1}\right) u$, where $u \in$ $H^{2}\left(\Omega_{\varepsilon}\right)^{3} \cap V_{\varepsilon}$ satisfies the Navier boundary conditions,

$$
\|w\|_{L^{\infty}} \leq C \varepsilon^{\frac{1}{2}}\|w\|_{H^{2}} .
$$

Proof. To show this estimate, we make a change of variables which transforms the domain $\Omega_{\varepsilon}$ into the domain $\Omega=\mathbb{T}^{3}$. We set:

$$
w\left(x_{1}, x_{2}, x_{3}\right)=w\left(y_{1}, y_{2}, \varepsilon g\left(y_{1}, y_{2}\right) y_{3}\right)=W\left(y_{1}, y_{2}, y_{3}\right), \text { for all }\left(y_{1}, y_{2}, y_{3}\right) \in \mathbb{T}^{3} .
$$

Using the classical anisotropic Agmon inequality on the fixed domain $\Omega$ (see [44], [3]), we may write,

$$
\|w\|_{L^{\infty}\left(\Omega_{\varepsilon}\right)}=\|W\|_{L^{\infty}(\Omega)} \leq c|W|_{L^{2}(\Omega)}^{1 / 4} \prod_{j=1}^{3}\left(|W|_{L^{2}(\Omega)}+\left|\frac{\partial W}{\partial y_{j}}\right|_{L^{2}(\Omega)}+\left|\frac{\partial^{2} W}{\partial y_{j}^{2}}\right|_{L^{2}(\Omega)}\right)^{1 / 4} .
$$

We next go back to the variable domain $\Omega_{\varepsilon}$. We notice that

$$
\frac{\partial W}{\partial y_{3}}=\varepsilon g \frac{\partial w}{\partial x_{3}}, \frac{\partial^{2} w}{\partial y_{3}^{2}}=\varepsilon^{2} g^{2} \frac{\partial^{2} w}{\partial x_{3}^{2}}
$$

whereas, for $j=1,2$, we have,

$$
\begin{aligned}
\frac{\partial W}{\partial y_{j}} & =\frac{\partial w}{\partial x_{j}}+\varepsilon \frac{\partial g}{\partial x_{j}} \frac{\partial w}{\partial x_{3}} \\
\frac{\partial^{2} W}{\partial y_{j}^{2}} & =\frac{\partial^{2} w}{\partial x_{j}^{2}}+2 \varepsilon \frac{\partial g}{\partial x_{j}} \frac{\partial^{2} w}{\partial x_{j} \partial x_{3}}+\varepsilon \frac{\partial^{2} g}{\partial x_{j}^{2}} \frac{\partial w}{\partial x_{3}}+\varepsilon^{2}\left(\frac{\partial g}{\partial x_{j}}\right)^{2} \frac{\partial^{2} w}{\partial x_{3}^{2}} .
\end{aligned}
$$

Using the formulas (3.34) and (3.35), we deduce from the inequality (3.33) that

$$
\|w\|_{L^{\infty}\left(\Omega_{\varepsilon}\right)} \leq C \varepsilon^{-\frac{1}{2}}\|w\|_{L^{2}\left(\Omega_{\varepsilon}\right)}^{\frac{1}{4}}\|w\|_{H^{2}\left(\Omega_{\varepsilon}\right)}^{\frac{1}{2}}\left(\|w\|_{L^{2}\left(\Omega_{\varepsilon}\right)}+\varepsilon\left\|\frac{\partial w}{\partial x_{3}}\right\|_{L^{2}\left(\Omega_{\varepsilon}\right)}+\varepsilon^{2}\left\|\frac{\partial^{2} w}{\partial x_{3}^{2}}\right\|_{L^{2}\left(\Omega_{\varepsilon}\right)}\right)^{\frac{1}{4}} .
$$

The inequality (3.36) and Proposition 3.6 at once imply that

$$
\|w\|_{L^{\infty}\left(\Omega_{\varepsilon}\right)} \leq \varepsilon^{1 / 2}\|w\|_{H^{2}\left(\Omega_{\varepsilon}\right)} .
$$

which proves the lemma.

We continue with a simple estimate on $v=M_{1} u$, where $u \in H^{2}\left(\Omega_{\varepsilon}\right)^{3} \cap V_{\varepsilon}$. We recall that $v_{h}=M_{h} u$ denotes the horizontal part of $v$.

Lemma 3.12. There exist positive constants $\varepsilon_{0}$ and $C$ such that, for any $0<\varepsilon \leq \varepsilon_{0}$, the following estimate holds for any $v=M_{1} u$, where $u \in H^{2}\left(\Omega_{\varepsilon}\right)^{3} \cap V_{\varepsilon}$

$$
\|v\|_{L^{4}} \leq C \varepsilon^{-\frac{1}{4}}\left\|v_{h}\right\|_{L^{2}}^{\frac{1}{2}}\left\|v_{h}\right\|_{H^{1}}^{\frac{1}{2}} \quad \text { and } \quad\|\nabla v\|_{L^{4}} \leq C \varepsilon^{-\frac{1}{4}}\left\|v_{h}\right\|_{H^{1}}^{\frac{1}{2}}\left\|v_{h}\right\|_{H^{2}}^{\frac{1}{2}} .
$$

Proof. We observe first that $|v| \leq C\left|v_{h}\right|$. Therefore, by the two-dimensional GagliardoNirenberg inequality we infer that

$$
\|v\|_{L^{4}} \leq C \varepsilon^{\frac{1}{4}}\left\|v_{h}\right\|_{L^{4}\left(\mathbb{T}^{2}\right)} \leq C \varepsilon^{\frac{1}{4}}\left\|v_{h}\right\|_{L^{2}\left(\mathbb{T}^{2}\right)}^{\frac{1}{2}}\left\|v_{h}\right\|_{H^{1}\left(\mathbb{T}^{2}\right)}^{\frac{1}{2}} \leq C \varepsilon^{-\frac{1}{4}}\left\|v_{h}\right\|_{L^{2}}^{\frac{1}{2}}\left\|v_{h}\right\|_{H^{1}}^{\frac{1}{2}} .
$$


The proof of the second inequality of (3.37), which uses $|\nabla v| \leq C\left(\left|v_{h}\right|+\left|\nabla_{h} v_{h}\right|\right)$, follows in the same way.

\section{EStimates FOR THE TRILINEAR TERM}

The various estimates and lemmas of the previous sections allow us to give the needed estimates concerning the trilinear term $\left|\int_{\Omega_{\varepsilon}} u \cdot \nabla u U d x\right|$ where $u \in H^{2}\left(\Omega_{\varepsilon}\right)^{3} \cap V_{\varepsilon}$ is a solution of the Navier-Stokes equations (1.2) which satisfies the Navier boundary conditions (1.3). In particular, the main objective of this section is to derive a "good" estimate of the term $\left|\int_{\Omega_{\varepsilon}} v \cdot \nabla u u d x\right|$ (see Proposition 4.3), where

$$
u=v+w, \quad \text { with } v=M_{1} u, \quad w=\left(I-M_{1}\right) .
$$

We recall that $v_{h}=M_{h} v$ denotes the horizontal part of $v$ (see (3.4)).

We point out that we get a good estimate of the cubic term $\left|\int_{\Omega_{\varepsilon}} u \cdot \nabla u u d x\right|$ by decomposing the vector $u$ into $v+w$ and by using the smallness properties of $w$ as well as the fact that $v$ depends only on the horizontal variable $x_{h}$. Here the decomposition $u=v+w$ is more convenient than the decomposition $u=M_{h} u+\left(I-M_{h}\right) u$, since $v$ and $w$ are divergence free and tangent to the boundary. Both properties are widely used in the computations below involving numerous integrations by parts. We begin with the following straightforward result.

Proposition 4.1. There exist positive constants $\varepsilon_{0}$ and $C$ such that, for $0<\varepsilon \leq \varepsilon_{0}$, for any $U \in L^{2}\left(\Omega_{\varepsilon}\right)^{3}$, any $U^{*} \in H^{1}\left(\Omega_{\varepsilon}\right)^{3}$, and any $w=\left(I-M_{1}\right) u$ with $u \in D\left(A_{\varepsilon}\right)$, we have,

$$
\left|\int_{\Omega_{\varepsilon}} w \cdot \nabla U^{*} U d x\right| \leq C \varepsilon^{1 / 2}\left\|U^{*}\right\|_{H^{1}}\|w\|_{H^{2}}\|U\|_{L^{2}}, \quad \text { for all } \varepsilon \in\left(0, \varepsilon_{0}\right]
$$

Proof. Applying the Hölder inequality and Lemma 3.11, we may write,

$$
\left|\int_{\Omega_{\varepsilon}} w \cdot \nabla U^{*} U d x\right| \leq\|w\|_{L^{\infty}}\left\|\nabla U^{*}\right\|_{L^{2}}\|U\|_{L^{2}} \leq \varepsilon^{1 / 2}\left\|U^{*}\right\|_{H^{1}}\|w\|_{H^{2}}\|U\|_{L^{2}},
$$

which proves the inequality (4.2).

Now we want to estimate the term $\left|\int_{\Omega_{\varepsilon}}(v \cdot \nabla u) \Delta u d x\right|$. Integrating this term by parts, we obtain,

$$
\begin{aligned}
\int_{\Omega_{\varepsilon}}(v \cdot \nabla u) \Delta u d x=-\sum_{i, j, k} \int_{\Omega_{\varepsilon}} v_{i} \partial_{i} \partial_{k} u_{j} \partial_{k} u_{j} d x & -\sum_{i, j, k} \int_{\Omega_{\varepsilon}} \partial_{k} v_{i} \partial_{i} u_{j} \partial_{k} u_{j} d x \\
& +\sum_{i, j, k} \int_{\Gamma_{0} \cup \Gamma_{\varepsilon}} v_{i} \partial_{i} u_{j} \partial_{k} u_{j} N_{k} d \sigma_{x} .
\end{aligned}
$$

We claim that the first term in the right hand side of (4.3) vanishes. Indeed, integrating by parts, using the facts that $v \cdot N=0$ on $\Gamma$ and that the divergence of $v$ vanishes in $\Omega_{\varepsilon}$, we obtain that

$$
\sum_{i, j, k} \int_{\Omega_{\varepsilon}} v_{i} \partial_{i} \partial_{k} u_{j} \partial_{k} u_{j} d x=-\frac{1}{2} \sum_{i, j, k} \int_{\Omega_{\varepsilon}} \partial_{i} v_{i}\left(\partial_{k} u_{j}\right)^{2} d x=0
$$


Thus (4.3) reduces to the equality

$$
\int_{\Omega_{\varepsilon}}(v \cdot \nabla u) \Delta u d x=-\sum_{i, j, k} \int_{\Omega_{\varepsilon}} \partial_{k} v_{i} \partial_{i} u_{j} \partial_{k} u_{j} d x+B_{0}
$$

where

$$
B_{0}=\sum_{i, j, k} \int_{\Gamma_{0} \cup \Gamma_{\varepsilon}} v_{i} \partial_{i} u_{j} \partial_{k} u_{j} N_{k} d \sigma_{x}
$$

We now estimate each term in the right hand side of (4.4) as follows. Using the decomposition (4.1), we obtain

$$
\begin{aligned}
\sum_{i, j, k} \int_{\Omega_{\varepsilon}} \partial_{k} v_{i} \partial_{i} u_{j} \partial_{k} u_{j} d x= & \sum_{i, j, k=1}^{2} \int_{\Omega_{\varepsilon}} \partial_{k} v_{i} \partial_{i} v_{j} \partial_{k} v_{j} d x+\int_{\Omega_{\varepsilon}}\left(\partial_{3} v_{3}\right)^{3} d x \\
& +\sum_{k=1}^{2} \int_{\Omega_{\varepsilon}} \partial_{k} v_{3} \partial_{3} v_{3} \partial_{k} v_{3} d x+\sum_{i, k=1}^{2} \int_{\Omega_{\varepsilon}} \partial_{k} v_{i} \partial_{i} v_{3} \partial_{k} v_{3} d x \\
& +\sum_{i, j, k} \int_{\Omega_{\varepsilon}} \partial_{k} v_{i}\left(\partial_{i} w_{j} \partial_{k} v_{j}+\partial_{i} v_{j} \partial_{k} w_{j}\right) d x \\
& +\sum_{i, j, k} \int_{\Omega_{\varepsilon}} \partial_{k} v_{i} \partial_{i} w_{j} \partial_{k} w_{j} d x \\
= & B_{1}+B_{2}+B_{3}+B_{4}+B_{5}+B_{6} .
\end{aligned}
$$

Next, it is straightforward to verify from the definition of $v$ that the integrands from $B_{2}$, $B_{3}$ and $B_{4}$ can be bounded pointwise by $C\left(\left|v_{h}\right||\nabla v|^{2}+\varepsilon|\nabla v|^{3}\right)$. Consequently, by using Lemma 3.12 and the Hölder inequality, we find

$$
\begin{aligned}
\left|B_{2}\right|+\left|B_{3}\right|+\left|B_{4}\right| \leq & C \int_{\Omega_{\varepsilon}}\left(\left|v_{h}\right|+\varepsilon|\nabla v|\right)|\nabla v|^{2} \leq C\left(\left\|v_{h}\right\|_{L^{2}}+\varepsilon\|v\|_{H^{1}}\right)\|\nabla v\|_{L^{4}}^{2} \\
& \leq C \varepsilon^{-1 / 2}\left\|v_{h}\right\|_{L^{2}}\left\|v_{h}\right\|_{H^{1}}\left\|v_{h}\right\|_{H^{2}}+C \varepsilon^{1 / 2}\left\|v_{h}\right\|_{H^{1}}^{2}\left\|v_{h}\right\|_{H^{2}},
\end{aligned}
$$

for $0<\varepsilon \leq \varepsilon_{0}$.

The estimate of the term $B_{5}$ also follows from Lemma 3.12, the Hölder inequality, and (3.14):

$$
\left|B_{5}\right| \leq C\|\nabla v\|_{L^{4}}^{2}\|\nabla w\|_{L^{2}} \leq C \varepsilon^{1 / 2}\left\|v_{h}\right\|_{H^{1}}\left\|v_{h}\right\|_{H^{2}}\|w\|_{H^{2}}, \quad \text { for all } \varepsilon \in\left(0, \varepsilon_{0}\right] .
$$

We next estimate the term $B_{6}$. According to (3.28) (with $q=4$ ), we may write

$$
\left|B_{6}\right| \leq C\|v\|_{H^{1}}\|\nabla w\|_{L^{4}}^{2} \leq C \varepsilon^{1 / 2}\|v\|_{H^{1}}\|w\|_{H^{2}}^{2}, \quad \text { for all } \varepsilon \in\left(0, \varepsilon_{0}\right] .
$$

The estimate of the term $B_{1}$ is given in the next proposition.

Proposition 4.2. There exist positive constants $\varepsilon_{0}$ and $C$, where $C$ does not depend on $\varepsilon$, such that, for $0<\varepsilon \leq \varepsilon_{0}$ and any $v=M_{1} u$ with $u \in H^{2}\left(\Omega_{\varepsilon}\right)^{3} \cap V_{\varepsilon}$, we have,

$$
\left|\sum_{i, j, k=1}^{2} \int_{\Omega_{\varepsilon}} \partial_{k} v_{i} \partial_{i} v_{j} \partial_{k} v_{j} d x\right| \leq C \varepsilon^{-1 / 2}\left\|v_{h}\right\|_{L^{2}}\left\|v_{h}\right\|_{H^{1}}\left\|v_{h}\right\|_{H^{2}} \quad \text { for all } \varepsilon \in\left(0, \varepsilon_{0}\right] \text {. }
$$


Proof. To prove (4.10), we note that the integrand does not depend on $x_{3}$ and therefore,

$$
\sum_{i, j, k=1}^{2} \int_{\Omega_{\varepsilon}} \partial_{k} v_{i} \partial_{i} v_{j} \partial_{k} v_{j} d x=\sum_{i, j, k=1}^{2} \int_{\mathbb{T}^{2}} \varepsilon g \partial_{k} v_{i} \partial_{i} v_{j} \partial_{k} v_{j} d x_{h}
$$

But, since $\operatorname{div}\left(g v_{h}\right)=0$ and thus $\operatorname{div}\left(v_{h}\right)=-(1 / g) v_{h} \cdot \nabla g$ (see (3.3)), an easy computation shows that,

$$
\begin{aligned}
\sum_{i, j, k=1}^{2} \int_{\mathbb{T}^{2}} \varepsilon g \partial_{k} v_{i} \partial_{i} v_{j} \partial_{k} v_{j} d x_{h}=\int_{\mathbb{T}^{2}} \varepsilon g \operatorname{div}\left(v_{h}\right)\left[\left(\partial_{2} v_{1}\right)^{2}+\left(\partial_{1} v_{2}\right)^{2}+\left(\partial_{1} v_{1}\right)^{2}\right. \\
\left.+\left(\partial_{2} v_{2}\right)^{2}+\partial_{2} v_{1} \partial_{1} v_{2}-\partial_{1} v_{1} \partial_{2} v_{2}\right] d x_{h} \\
=-\int_{\mathbb{T}^{2}} \varepsilon v_{h} \cdot \nabla g\left[\left(\partial_{2} v_{1}\right)^{2}+\left(\partial_{1} v_{2}\right)^{2}+\left(\partial_{1} v_{1}\right)^{2}\right. \\
\left.+\left(\partial_{2} v_{2}\right)^{2}+\partial_{2} v_{1} \partial_{1} v_{2}-\partial_{1} v_{1} \partial_{2} v_{2}\right] d x_{h}
\end{aligned}
$$

We infer from (4.11) and the two-dimensional Gagliardo-Nirenberg inequality (see [27]) that

$$
\begin{aligned}
\sum_{i, j, k=1}^{2} \int_{\Omega_{\varepsilon}} \partial_{k} v_{i} \partial_{i} v_{j} \partial_{k} v_{j} d x & \leq C \varepsilon\left\|v_{h}\right\|_{L^{2}\left(\mathbb{T}^{2}\right)}\left\|\nabla v_{h}\right\|_{L^{4}\left(\mathbb{T}^{2}\right)}^{2} \\
& \leq C \varepsilon^{-1 / 2}\left\|v_{h}\right\|_{L^{2}}\left\|v_{h}\right\|_{H^{1}}\left\|v_{h}\right\|_{H^{2}}
\end{aligned}
$$

which completes the proof of Proposition 4.2.

Since we have estimated the terms $B_{i}, i=1,2,3,4,5,6$, it remains to bound the integral term $B_{0}$ defined in (4.5):

$$
B_{0}=\sum_{i, j, k} \int_{\Gamma_{0} \cup \Gamma_{\varepsilon}} v_{i} \partial_{i} u_{j} \partial_{k} u_{j} N_{k} d \sigma_{x}
$$

Note that the term $\sum_{i, j, k} \int_{\Gamma_{0}} v_{i} \partial_{i} u_{j} \partial_{k} u_{j} N_{k} d \sigma_{x}$ vanishes, due to the Navier boundary conditions on the boundary $\Gamma_{0}$. Moreover, on the boundary part $\Gamma_{\varepsilon}, N_{k}$ coincides with $\widetilde{N}_{k}$. Furthermore, in view of (3.11), on $\Gamma_{\varepsilon}$ we have the equality $d \sigma_{x}=\sqrt{1+\varepsilon^{2}|\nabla g|^{2}} d x_{h}$. For the sake of conciseness, we will use the notation

$$
d \widetilde{x}_{h}=\sqrt{1+\varepsilon^{2}\left|\nabla g\left(x_{h}\right)\right|^{2}} d x_{h}
$$

in the sequel. Since the quantity $\sqrt{1+\varepsilon^{2}|\nabla g|^{2}}$ is of order of one, when $\varepsilon$ is small, we see that $d \widetilde{x}_{h}$ is quite similar to $d x_{h}$, and will be treated likewise. Next, according to the equalities (3.17) and (3.19), we have,

$$
\partial_{3} u_{j}=x_{3} H_{\varepsilon}^{j}, \quad \partial_{j} u_{3}=x_{3} F_{\varepsilon}^{j}, \quad \text { on } \Gamma_{\varepsilon}, \text { for } j=1,2,
$$


where $H_{\varepsilon}^{j}=H_{\varepsilon}^{j}(u, g)$ and $F_{\varepsilon}^{j}=F_{\varepsilon}^{j}(u, g)$ satisfy the estimates (3.20). In view of the remarks above, one has that

$$
\begin{aligned}
B_{0}= & \left.\sum_{i, j, k} \int_{\mathbb{T}^{2}}\left(v_{i} \partial_{i} u_{j} \partial_{k} u_{j} \widetilde{N}_{k}\right)\right|_{x_{3}=\varepsilon g} d \widetilde{x}_{h} \\
= & \left.\sum_{i \neq 3, j, k \neq 3} \int_{\mathbb{T}^{2}}\left(v_{i} \partial_{i} u_{j} \partial_{k} u_{j} \widetilde{N}_{k}\right)\right|_{x_{3}=\varepsilon g} d \widetilde{x}_{h}+\left.\sum_{i \neq 3, j \neq 3} \int_{\mathbb{T}^{2}}\left(v_{i} \partial_{i} u_{j} x_{3} H_{\varepsilon}^{j} \widetilde{N}_{3}\right)\right|_{x_{3}=\varepsilon g} d \widetilde{x}_{h} \\
& +\left.\sum_{i \neq 3} \int_{\mathbb{T}^{2}}\left(v_{i} x_{3} F_{\varepsilon}^{i} \partial_{3} u_{3} \widetilde{N}_{3}\right)\right|_{x_{3}=\varepsilon g} d \widetilde{x}_{h}+\left.\sum_{j, k} \int_{\mathbb{T}^{2}}\left(x_{3} g^{-1} v_{h} \cdot \nabla g \partial_{3} u_{j} \partial_{k} u_{j} \widetilde{N}_{k}\right)\right|_{x_{3}=\varepsilon g} d \widetilde{x}_{h} \\
= & B_{01}+B_{02}+B_{03}+B_{04} .
\end{aligned}
$$

To estimate the term $B_{01}$, we note that $v_{i} \partial_{i} u_{j} \partial_{k} u_{j} \widetilde{N}_{k}$ vanishes for $x_{3}=0$ and write:

$$
\begin{aligned}
B_{01}= & \sum_{i \neq 3, j, k \neq 3} \int_{\mathbb{T}^{2}} \int_{0}^{\varepsilon g} v_{i} \partial_{3}\left(\partial_{i} u_{j} \partial_{k} u_{j} \widetilde{N}_{k}\right) d x_{3} d \widetilde{x}_{h} \\
= & \sum_{i \neq 3, j, k \neq 3} \int_{\mathbb{T}^{2}} \int_{0}^{\varepsilon g} v_{i} \partial_{i} \partial_{3} u_{j} \partial_{k} u_{j} \widetilde{N}_{k} d x_{3} d \widetilde{x}_{h} \\
& +\sum_{i \neq 3, j, k \neq 3} \int_{\mathbb{T}^{2}} \int_{0}^{\varepsilon g} v_{i} \partial_{i} u_{j} \partial_{k} \partial_{3} u_{j} \widetilde{N}_{k} d x_{3} d \widetilde{x}_{h} \\
& +\sum_{i \neq 3, j, k \neq 3} \int_{\mathbb{T}^{2}} \int_{0}^{\varepsilon g} v_{i} \partial_{i} u_{j} \partial_{k} u_{j} \partial_{3} \widetilde{N}_{k} d x_{3} d \widetilde{x}_{h} \\
= & B_{011}+B_{012}+B_{013} .
\end{aligned}
$$

To estimate the term $B_{011}$ we remark that $\widetilde{N}_{k}$, for $k \neq 3$, is of order $\varepsilon$ and we use Lemma $3.5,(3.28)$ and (3.37) to obtain, for $0<\varepsilon \leq \varepsilon_{0}$,

$$
\begin{aligned}
\left|B_{011}\right| & \leq C \varepsilon\|v\|_{L^{4}}\|u\|_{H^{2}}\|\nabla u\|_{L^{4}} \\
& \leq C \varepsilon^{3 / 4}\left\|v_{h}\right\|_{L^{2}}^{\frac{1}{2}}\left\|v_{h}\right\|_{H^{1}}^{\frac{1}{2}}\|u\|_{H^{2}}\left(\|\nabla v\|_{L^{4}}+\varepsilon^{1 / 4}\|w\|_{H^{2}}\right) \leq C \varepsilon^{1 / 2}\|u\|_{H^{1}}\|u\|_{H^{2}}^{2} .
\end{aligned}
$$

Proceeding exactly in the same way for the term $B_{012}$, we obtain the estimate

$$
\left|B_{011}\right|+\left|B_{012}\right| \leq C \varepsilon^{\frac{1}{2}}\|u\|_{H^{1}}\|u\|_{H^{2}}^{2}, \quad \text { for all } \varepsilon \in\left(0, \varepsilon_{0}\right] .
$$

We next estimate the term $B_{013}$. We note that

$$
\begin{aligned}
B_{013}= & \sum_{i \neq 3, j, k \neq 3} \int_{\mathbb{T}^{2}} \int_{0}^{\varepsilon g} v_{i}\left(\partial_{i} w_{j} \partial_{k} v_{j}+\partial_{i} v_{j} \partial_{k} w_{j}\right) \partial_{3} \widetilde{N}_{k} d x_{3} d \widetilde{x}_{h} \\
& +\sum_{i \neq 3, j, k \neq 3} \int_{\mathbb{T}^{2}} \int_{0}^{\varepsilon g} v_{i} \partial_{i} w_{j} \partial_{k} w_{j} \partial_{3} \widetilde{N}_{k} d x_{3} d \widetilde{x}_{h} \\
& +\sum_{i \neq 3, j, k \neq 3} \int_{\mathbb{T}^{2}} \int_{0}^{\varepsilon g} v_{i} \partial_{i} v_{j} \partial_{k} v_{j} \partial_{3} \widetilde{N}_{k} d x_{3} d \widetilde{x}_{h} .
\end{aligned}
$$


Applying Lemma 3.10, we at once get, for $0<\varepsilon \leq \varepsilon_{0}$,

$$
\begin{aligned}
\left|\sum_{i \neq 3, j, k \neq 3} \int_{\mathbb{T}^{2}} \int_{0}^{\varepsilon g} v_{i} \partial_{i} w_{j} \partial_{k} w_{j} \partial_{3} \widetilde{N}_{k} d x_{3} d \widetilde{x}_{h}\right| & \leq C\|v\|_{L^{2}}\|\nabla w\|_{L^{4}}^{2} \\
& \leq C \varepsilon^{1 / 2}\|v\|_{L^{2}}\|u\|_{H^{2}}^{2}
\end{aligned}
$$

As argued in the passage from (4.11) to (4.12), and using (3.37), we get the following estimate

$$
\left|\sum_{i \neq 3, j, k \neq 3} \int_{\mathbb{T}^{2}} \int_{0}^{\varepsilon g} v_{i} \partial_{i} v_{j} \partial_{k} v_{j} \partial_{3} \widetilde{N}_{k} d x_{3} d \widetilde{x}_{h}\right| \leq C \varepsilon^{-1 / 2}\left\|v_{h}\right\|_{L^{2}}\left\|v_{h}\right\|_{H^{1}}\left\|v_{h}\right\|_{H^{2}} .
$$

Next, from Lemma 3.5, (3.14) and (3.37), we get

$$
\begin{gathered}
\left|\sum_{i \neq 3, j, k \neq 3} \int_{\mathbb{T}^{2}} \int_{0}^{\varepsilon g} v_{i}\left(\partial_{i} w_{j} \partial_{k} v_{j}+\partial_{i} v_{j} \partial_{k} w_{j}\right) \partial_{3} \widetilde{N}_{k} d x_{3} d \widetilde{x}_{h}\right| \leq C\|v\|_{L^{4}}\|\nabla v\|_{L^{4}}\|w\|_{H^{1}} \\
\leq C \varepsilon^{1 / 2}\left\|v_{h}\right\|_{L^{2}}^{1 / 2}\left\|v_{h}\right\|_{H^{1}}\left\|v_{h}\right\|_{H^{2}}^{1 / 2}\|w\|_{H^{2}} \leq C \varepsilon^{1 / 2}\|u\|_{H^{1}}\|u\|_{H^{2}}^{2} .
\end{gathered}
$$

The relations (4.14), (4.15), (4.16), (4.17) and (4.18) imply that

$$
\left|B_{01}\right| \leq C\left(\varepsilon^{1 / 2}\|u\|_{H^{1}}\|u\|_{H^{2}}^{2}+\varepsilon^{-1 / 2}\left\|v_{h}\right\|_{L^{2}}\left\|v_{h}\right\|_{H^{1}}\left\|v_{h}\right\|_{H^{2}}\right) .
$$

We next estimate the term $B_{02}$ from (4.13). We first write $B_{02}$ as

$$
\begin{aligned}
B_{02}= & \sum_{i \neq 3, j \neq 3} \int_{\mathbb{T}^{2}} \int_{0}^{\varepsilon g} \partial_{3}\left(v_{i} \partial_{i} u_{j} x_{3} H_{\varepsilon}^{j} \widetilde{N}_{3}\right) d x_{3} d \widetilde{x}_{h} \\
= & \sum_{i \neq 3, j \neq 3} \int_{\mathbb{T}^{2}} \int_{0}^{\varepsilon g} x_{3} v_{i}\left(\partial_{i} \partial_{3} u_{j} H_{\varepsilon}^{j} \widetilde{N}_{3}+\partial_{i} u_{j} \partial_{3} H_{\varepsilon}^{j} \widetilde{N}_{3}+\partial_{i} u_{j} H_{\varepsilon}^{j} \partial_{3} \widetilde{N}_{3}\right) d x_{3} d \widetilde{x}_{h} \\
& +\sum_{i \neq 3, j \neq 3} \int_{\mathbb{T}^{2}} \int_{0}^{\varepsilon g} v_{i} \partial_{i} u_{j} H_{\varepsilon}^{j} \widetilde{N}_{3} d x_{3} d \widetilde{x}_{h} \\
& :=B_{021}+B_{022} .
\end{aligned}
$$

Since $x_{3} \leq C \varepsilon, \widetilde{N}_{3} \leq C$, and $\partial_{3} \widetilde{N}_{3} \leq C \varepsilon$, we obtain, by using the estimates (3.20),

$$
\left|B_{021}\right| \leq C \varepsilon\left\|v_{h}\right\|_{L^{\infty}}\|u\|_{H^{1}}\|u\|_{H^{2}} \leq C \varepsilon^{1 / 2}\left\|v_{h}\right\|_{H^{2}}\|u\|_{H^{1}}\|u\|_{H^{2}}
$$

To estimate $B_{022}$ we observe from the explicit formula for $H_{\varepsilon}^{j}$ that $\left|H_{\varepsilon}^{j}\right| \leq C(|u|+|\nabla u|)$ pointwise, so by using (3.27), (3.28) and (3.37), we find

$$
\begin{aligned}
\left|B_{022}\right| & \leq C \int_{\Omega_{\varepsilon}}\left|v_{h}\right|\left(|u|^{2}+|\nabla u|^{2}\right) \\
& \leq C\left\|v_{h}\right\|_{L^{2}}\left(\|u\|_{L^{4}}^{2}+\|\nabla u\|_{L^{4}}^{2}\right) \\
& \leq C\left\|v_{h}\right\|_{L^{2}}\left(\|v\|_{L^{4}}^{2}+\|\nabla v\|_{L^{4}}^{2}+\|w\|_{L^{4}}^{2}+\|\nabla w\|_{L^{4}}^{2}\right) \\
& \leq C\left\|v_{h}\right\|_{L^{2}}\left(\varepsilon^{-\frac{1}{2}}\left\|v_{h}\right\|_{H^{1}}\left\|v_{h}\right\|_{H^{2}}+\varepsilon^{\frac{1}{2}} \mid w \|_{H^{2}}^{2}\right)
\end{aligned}
$$

The relations (4.20), (4.21) and (4.22) then imply that

$$
\left|B_{02}\right| \leq C \varepsilon^{1 / 2}\|u\|_{H^{1}}\|u\|_{H^{2}}^{2}+C \varepsilon^{-1 / 2}\left\|v_{h}\right\|_{L^{2}}\left\|v_{h}\right\|_{H^{1}}\left\|v_{h}\right\|_{H^{2}} .
$$


The term $B_{03}$ from (4.13) is entirely similar to $B_{02}$ so the same estimate holds true for $B_{03}$. It remains to estimate the term $B_{04}$. We first write:

$$
\begin{aligned}
B_{04}= & \sum_{j, k} \int_{\mathbb{T}^{2}} \int_{0}^{\varepsilon g} \partial_{3}\left(x_{3} g^{-1} v_{h} \cdot \nabla g \partial_{3} u_{j} \partial_{k} u_{j} \widetilde{N}_{k}\right) d x_{3} d \widetilde{x}_{h} \\
= & \sum_{j, k} \int_{\mathbb{T}^{2}} g^{-1} v_{h} \cdot \nabla g \int_{0}^{\varepsilon g} x_{3} \partial_{3}\left(\partial_{3} u_{j} \partial_{k} u_{j} \widetilde{N}_{k}\right) d x_{3} d \widetilde{x}_{h} \\
& \quad+\sum_{j, k} \int_{\mathbb{T}^{2}} g^{-1} v_{h} \cdot \nabla g \int_{0}^{\varepsilon g} \partial_{3} u_{j} \partial_{k} u_{j} \widetilde{N}_{k} d x_{3} d \widetilde{x}_{h} \\
= & B_{041}+B_{042} .
\end{aligned}
$$

We notice that in $B_{041}$, the terms corresponding to $k=3$ are of the same size as $B_{01}$, whereas the terms corresponding to $k=1,2$ are even smaller than the terms in $B_{01}$ (they are of the order of magnitude of $\left.\left|B_{01}\right| \times \varepsilon\right)$. For this reason, $B_{041}$ can be bounded like $B_{01}$ and we do not repeat the arguments. Likewise, the integrand in $B_{042}$ can be bounded pointwise by $C|v|_{h}|\nabla u|^{2}$, so the same estimates as in (4.22) can be performed for this term.

In the next proposition, we summarize all the estimates that we just have performed. Due to (4.4), (4.6) and (4.13), we have to take into account the estimates (4.7), (4.8), (4.9), (4.10), (4.19), (4.23), and Lemma 3.5 to obtain the following result.

Proposition 4.3. There exist positive constants $\varepsilon_{0}$ and $C$, where $C$ does not depend on $\varepsilon$, such that, for $0<\varepsilon \leq \varepsilon_{0}$, for any $u \in D\left(A_{\varepsilon}\right)$, with $v=M_{1} u$, we have,

$$
\left|\int_{\Omega_{\varepsilon}}(v \cdot \nabla u) \Delta u d x\right| \leq C \varepsilon^{1 / 2}\|u\|_{H^{1}}\|u\|_{H^{2}}\left(\|u\|_{H^{2}}+\varepsilon^{-1}\|u\|_{L^{2}}\right), \quad \text { for all } \varepsilon \in\left(0, \varepsilon_{0}\right] \text {. }
$$

\section{Global existence of Strong solutions}

Our main goal in this section is to prove Theorem 1.1. More precisely, we want to show that there exist positive constants $\varepsilon_{0}, \kappa_{0}, \kappa_{1}, K_{0}$ and $K_{1}$ such that, if $0<\varepsilon \leq \varepsilon_{0}$,

$$
\begin{aligned}
& \|u(0)\|_{H^{1}}^{2} \leq \kappa_{1}^{2} \varepsilon^{-1}, \quad\left\|v_{h}(0)\right\|_{L^{2}}^{2} \leq \kappa_{0}^{2} \\
& \|f\|_{L^{\infty}\left(L^{2}\right)}^{2} \leq K_{1}^{2} \varepsilon^{-1}, \quad\left\|M_{h} f\right\|_{L^{\infty}\left(L^{2}\right)}^{2} \leq K_{0}^{2},
\end{aligned}
$$

then the Navier-Stokes equations (1.2) have a unique global strong solution $u=u(t)$ with

$$
u \in C^{0}\left([0, \infty), V_{\varepsilon}\right) \cap L^{\infty}\left([0, \infty), V_{\varepsilon}\right) \cap L_{l o c}^{2}\left((0, \infty), H^{2}\left(\Omega_{\varepsilon}\right)\right) \cap H_{l o c}^{1}\left((0, \infty), H_{\varepsilon}\right) .
$$

We notice that, since $f=\mathbb{P}_{\varepsilon} f+\nabla q$ and that we can replace $\nabla p$ by $\nabla p+\nabla q$ in the equation (1.2), we may assume without loss of generality that $f=\mathbb{P}_{\varepsilon} f$.

For simplicity, we assume in the sequel that the viscosity $\nu$ is equal to one. Except for some straightforward changes in the notation, the same argument applies for any positive $\nu$ (of course, some of the constants will depend on $\nu$ ). The constant $C$ denotes a generic constant independent of $\varepsilon$ whose value may change from one line to another. All other constants are fixed once and for all.

In this section, $u=u(t)$ will denote the unique, maximally defined, strong solution of (1.2), with initial data $u_{0} \in V_{\varepsilon}$ and forcing term $f$, satisfying the conditions (5.1). According to Section 2.3, there exists a time $T \leq+\infty$ such that $u$ is a strong solution of 
(1.2) on the time interval $[0, T)$. Our purpose is to show that $T$ is actually equal to $+\infty$. Assume by contradiction that $T<+\infty$.

In the proof below, we again need to write the vector $u$ as a sum of two vectors, one of them depending only on the horizontal variable $x_{h}$ and the other one satisfying a Poincaré type inequality. In Section 4, it was convenient to choose the decomposition $u=M_{1} u+$ $\left(I-M_{1}\right) u \equiv v+w$. In this section, the decomposition

$$
u=M_{h} u+\left(I-M_{h}\right) u \equiv v_{h}+w^{*}
$$

seems to be more appropriate, since $M_{h}$ is an orthogonal projection in $L^{2}\left(\Omega_{\varepsilon}\right)$.

By taking the scalar product in $L^{2}\left(\Omega_{\varepsilon}\right)$ of (1.2) with $u$ and applying the Green formula (2.17), we obtain,

$$
\left(\frac{\mathrm{d}}{\mathrm{d} t} u, u\right)_{L^{2}}+\frac{1}{2} E(u, u)=(f, u)_{L^{2}} .
$$

Since $u$ belongs to $L^{2}\left((0, T), V_{\varepsilon}\right) \cap H^{1}\left((0, T), V_{\varepsilon}^{\prime}\right)$, by Lemma 1.2 of Chapter 3 of [42], we deduce from the above equality that

$$
\frac{1}{2} \frac{\mathrm{d}}{\mathrm{d} t}\|u\|_{L^{2}}^{2}+\frac{1}{2} E(u, u)=(f, u)_{L^{2}} .
$$

As $M_{h}$ is an orthogonal projection on $L^{2}\left(\Omega_{\varepsilon}\right)^{3}$, one has

$$
(f, u)_{L^{2}}=\left(\left(I-M_{h}\right) f,\left(I-M_{h}\right) u\right)_{L^{2}}+\left(M_{h} f, M_{h} u\right)_{L^{2}} .
$$

By using the Korn inequality (2.2), Lemma 3.5, and Proposition 3.6, we deduce from the equation (5.2) that there exists a constant $C$, which does not depend on $\varepsilon$, such that, for $0<\varepsilon \leq 1$, we have

$$
\begin{aligned}
\frac{1}{2} \frac{\mathrm{d}}{\mathrm{d} t}\|u\|_{L^{2}}^{2}+\frac{c_{0}}{2}\|u\|_{H^{1}}^{2} & \leq\left|\left(\left(I-M_{h}\right) f,\left(I-M_{h}\right) u\right)\right|+\left|\left(M_{h} f, M_{h} u\right)\right| \\
& \leq\left\|w^{*}\right\|_{L^{2}}\left\|\left(I-M_{h}\right) f\right\|_{L^{2}}+\left\|v_{h}\right\|_{L^{2}}\left\|M_{h} f\right\|_{L^{2}} \\
& \leq C\left[\varepsilon\|u\|_{H^{1}}\left\|\left(I-M_{h}\right) f\right\|_{L^{2}}+\|u\|_{L^{2}}\left\|M_{h} f\right\|_{L^{2}}\right] .
\end{aligned}
$$

By using the Young inequality, we find that there exists a positive constant $c_{1}$, independent of $\varepsilon$, such that, for $0<\alpha \leq c_{0} / 8$, and, for $0<\varepsilon \leq 1$,

$$
\frac{\mathrm{d}}{\mathrm{d} t}\|u\|_{L^{2}}^{2}+\alpha\|u\|_{L^{2}}^{2}+\frac{c_{0}}{2}\|u\|_{H^{1}}^{2} \leq c_{1}\left(\left\|M_{h} f\right\|_{L^{2}}^{2}+\varepsilon^{2}\|f\|_{L^{2}}^{2}\right) .
$$

Multiplying (5.3) by $\exp (\alpha s)$ and integrating the result between 0 and $t$, we obtain, for $t \in[0, T)$,

$$
\begin{gathered}
\|u(t)\|_{L^{2}}^{2}+\frac{c_{0}}{2} \int_{0}^{t} \exp (\alpha(s-t))\|u(s)\|_{H^{1}}^{2} d s \leq \exp (-\alpha t)\|u(0)\|_{L^{2}}^{2} \\
+\frac{C}{\alpha}\left(1-e^{-\alpha t}\right)\left(\left\|M_{h} f\right\|_{L^{\infty}\left(L^{2}\right)}^{2}+\varepsilon^{2}\|f\|_{L^{\infty}\left(L^{2}\right)}^{2}\right) .
\end{gathered}
$$

We next take the scalar product of the equations (1.2) with $A_{\varepsilon} u=-\mathbb{P}_{\varepsilon} \Delta u$. We point out that $A_{\varepsilon}^{1 / 2} u$ and $\frac{\mathrm{d}}{\mathrm{d} t} A_{\varepsilon}^{1 / 2} u$ belong to $L^{2}\left((0, T), V_{\varepsilon}\right)$ and to $L^{2}\left((0, T), V_{\varepsilon}^{\prime}\right)$ respectively, and thus, by (2.18) and by Lemma 1.2 of Chapter 3 of [42], we have

$$
\frac{1}{2} \frac{\mathrm{d}}{\mathrm{d} t} E(u, u)=\frac{\mathrm{d}}{\mathrm{d} t}\left\|A_{\varepsilon}^{1 / 2} u\right\|_{L^{2}}^{2}=2\left(\frac{\mathrm{d}}{\mathrm{d} t} u, A_{\varepsilon} u\right)_{L^{2}} .
$$


Taking the scalar product in $L^{2}\left(\Omega_{\varepsilon}\right)$ of (1.2) with $-\mathbb{P}_{\varepsilon} \Delta u$, applying the Green formula (2.17), and taking into account the above equality, we obtain,

$$
\frac{1}{4} \frac{\mathrm{d}}{\mathrm{d} t} E(u, u)+\left\|\mathbb{P}_{\varepsilon} \Delta u(t)\right\|_{L^{2}}^{2}=\int_{\Omega_{\varepsilon}}(u \cdot \nabla u) \mathbb{P}_{\varepsilon} \Delta u d x-\int_{\Omega_{\varepsilon}} f \cdot \mathbb{P}_{\varepsilon} \Delta u d x
$$

Writing the decomposition

$$
\int_{\Omega_{\varepsilon}}(u \cdot \nabla u) \mathbb{P}_{\varepsilon} \Delta u d x=\int_{\Omega_{\varepsilon}}(v \cdot \nabla u) \mathbb{P}_{\varepsilon} \Delta u d x+\int_{\Omega_{\varepsilon}}(w \cdot \nabla u) \mathbb{P}_{\varepsilon} \Delta u d x
$$

and applying Proposition 4.1 together with the Young inequality, we obtain

$$
\left|\int_{\Omega_{\varepsilon}}(u \cdot \nabla u) \mathbb{P}_{\varepsilon} \Delta u d x\right| \leq\left|\int_{\Omega_{\varepsilon}}(v \cdot \nabla u) \mathbb{P}_{\varepsilon} \Delta u d x\right|+\frac{1}{8}\left\|\mathbb{P}_{\varepsilon} \Delta u\right\|_{L^{2}}^{2}+C \varepsilon\|u\|_{H^{1}}^{2}\|w\|_{H^{2}}^{2} .
$$

Therefore, (5.5) and the Young inequality imply that

$$
\frac{1}{4} \frac{\mathrm{d}}{\mathrm{d} t} E(u, u)+\frac{3}{4}\left\|\mathbb{P}_{\varepsilon} \Delta u(t)\right\|_{L^{2}}^{2} \leq\left|\int_{\Omega_{\varepsilon}}(v \cdot \nabla u) \mathbb{P}_{\varepsilon} \Delta u d x\right|+C \varepsilon\|u\|_{H^{1}}^{2}\|w\|_{H^{2}}^{2}+2\|f\|_{L^{2}}^{2},
$$

or also, by Proposition 3.9,

$$
\begin{aligned}
\frac{1}{4} \frac{\mathrm{d}}{\mathrm{d} t} E(u(t), u(t))+\frac{3}{8}\|\Delta u(t)\|_{L^{2}}^{2} \leq & \left|\int_{\Omega_{\varepsilon}}(v \cdot \nabla u) \mathbb{P}_{\varepsilon} \Delta u d x\right|+C \varepsilon\|u(t)\|_{H^{1}}^{2}\|w(t)\|_{H^{2}}^{2} \\
& +C\|u(t)\|_{H^{1}}^{2}+C \varepsilon^{2}\|u(t)\|_{H^{2}}^{2}+2\|f(t)\|_{L^{2}}^{2} .
\end{aligned}
$$

It remains to estimate the term $\left|\int_{\Omega_{\varepsilon}}(v \cdot \nabla u) \mathbb{P}_{\varepsilon} \Delta u d x\right|$. Using the decomposition

$$
\int_{\Omega_{\varepsilon}}(v \cdot \nabla u) \mathbb{P}_{\varepsilon} \Delta u d x=\int_{\Omega_{\varepsilon}}(v \cdot \nabla u)\left(\mathbb{P}_{\varepsilon}-I d\right) \Delta u d x+\int_{\Omega_{\varepsilon}}(v \cdot \nabla u) \Delta u d x
$$

and applying Propositions 3.9 and 4.3, we get

$$
\begin{aligned}
& \int_{\Omega_{\varepsilon}}(v \cdot \nabla u) \mathbb{P}_{\varepsilon} \Delta u d x \leq C\|v \nabla u\|_{L^{2}}\left(\|u\|_{H^{1}}+\varepsilon\|u\|_{H^{2}}\right)+\left|\int_{\Omega_{\varepsilon}}(v \cdot \nabla u) \Delta u d x\right| \\
& \leq C\left(\|v \nabla u\|_{L^{2}}\left(\|u\|_{H^{1}}+\varepsilon\|u\|_{H^{2}}\right)+\varepsilon^{1 / 2}\|u\|_{H^{1}}\|u\|_{H^{2}}\left(\|u\|_{H^{2}}+\varepsilon^{-1}\|u\|_{L^{2}}\right)\right) .
\end{aligned}
$$

Writing again $\nabla u=\nabla v+\nabla w$, applying Lemmas 3.10 and 3.12, and using the interpolation inequality

$$
\|v\|_{H^{1}} \leq C\|v\|_{L^{2}}^{\frac{1}{2}}\|v\|_{H^{2}}^{\frac{1}{2}} \leq C\|u\|_{L^{2}}^{\frac{1}{2}}\|u\|_{H^{2}}^{\frac{1}{2}}
$$

we obtain,

$$
\begin{aligned}
\|v \nabla u\|_{L^{2}}\left(\|u\|_{H^{1}}+\varepsilon\right. & \left.\|u\|_{H^{2}}\right) \\
& \leq C\|v\|_{L^{4}}\left(\|\nabla v\|_{L^{4}}+\varepsilon^{\frac{3}{4}-\frac{1}{2}}\|w\|_{H^{2}}\right)\left(\|u\|_{H^{1}}+\varepsilon\|u\|_{H^{2}}\right) \\
& \leq C\left(\varepsilon^{-\frac{1}{2}}\|u\|_{L^{2}}\|u\|_{H^{2}}+\|u\|_{L^{2}}^{\frac{1}{2}}\|u\|_{H^{1}}^{\frac{1}{2}}\|u\|_{H^{2}}\right)\left(\|u\|_{H^{1}}+\varepsilon\|u\|_{H^{2}}\right) .
\end{aligned}
$$


The estimates (5.6) to (5.7) imply that, for $0<\varepsilon \leq \varepsilon_{0}$,

$$
\begin{aligned}
\frac{1}{4} \frac{\mathrm{d}}{\mathrm{d} t} E(u, u)+\frac{3}{8}\|\Delta u(t)\|_{L^{2}}^{2} \leq & 2\|f\|_{L^{2}}^{2}+C\|u\|_{H^{1}}^{2}+C \varepsilon^{2}\|u\|_{H^{2}}^{2} \\
& +C \varepsilon^{1 / 2}\|u\|_{H^{1}}\|u\|_{H^{2}}^{2}+C \varepsilon\|u\|_{H^{1}}^{2}\|u\|_{H^{2}}^{2} \\
& +C\|u\|_{L^{2}}^{1 / 2}\|u\|_{H^{1}}^{3 / 2}\|u\|_{H^{2}}+C \varepsilon^{-1 / 2}\|u\|_{L^{2}}\|u\|_{H^{1}}\|u\|_{H^{2}} .
\end{aligned}
$$

Using the Young inequality several times, we at once infer from Proposition 3.7 and the above estimate (5.8), that there exist positive constants $\beta_{0}, C_{0}$ and $C$, which are independent of $\varepsilon$, such that, for $0<\varepsilon \leq \varepsilon_{0}$,

$$
\begin{aligned}
\frac{\mathrm{d}}{\mathrm{d} t} E(u, u)+\left(\beta_{0}-C_{0} \varepsilon^{1 / 2}\right. & \left.\|u\|_{H^{1}}-C_{0} \varepsilon\|u\|_{H^{1}}^{2}-C_{0} \varepsilon^{2}\right)\|u\|_{H^{2}}^{2} \\
& \leq C\left(\|u\|_{H^{1}}^{2}+\|f\|_{L^{2}}^{2}+\varepsilon^{-1}\|u\|_{L^{2}}^{2}\|u\|_{H^{1}}^{2}+\|u\|_{L^{2}}\|u\|_{H^{1}}^{3}\right)
\end{aligned}
$$

To prove global existence of the solution $u$, we argue by contradiction. We assume that $\varepsilon_{0} \leq \sqrt{\frac{\beta_{0}}{2 C_{0}}}$ and that, for $0<\varepsilon \leq \varepsilon_{0}$, the initial data $u_{0}$ satisfy the following condition

$$
\frac{\beta_{0}}{2}>C_{0} \varepsilon^{1 / 2}\|u(0)\|_{H^{1}}+C_{0} \varepsilon\|u(0)\|_{H^{1}}^{2}+C_{0} \varepsilon^{2}
$$

Next, we assume that there exists a time $T_{0}>0$ such that,

$$
\begin{aligned}
& \frac{\beta_{0}}{2}>C_{0} \varepsilon^{1 / 2}\|u(t)\|_{H^{1}}+C_{0} \varepsilon\|u(t)\|_{H^{1}}^{2}+C_{0} \varepsilon^{2}, \quad \text { for all } t \in\left[0, T_{0}\right), \text { and } \\
& \frac{\beta_{0}}{2}=C_{0} \varepsilon^{1 / 2}\left\|u\left(T_{0}\right)\right\|_{H^{1}}+C_{0} \varepsilon\left\|u\left(T_{0}\right)\right\|_{H^{1}}^{2}+C_{0} \varepsilon^{2},
\end{aligned}
$$

and show that this leads to a contradiction.

Using the inequality (2.2), we deduce from (5.9) and (5.11) that, for $0<\varepsilon \leq \varepsilon_{0}$ and $t \in\left[0, T_{0}\right]$

$$
\begin{aligned}
\frac{\mathrm{d}}{\mathrm{d} t} E(u, u)+\beta E(u, u)+ & \frac{\beta_{0}}{4}\|u\|_{H^{2}}^{2} \\
& \leq C\left(\|u\|_{H^{1}}^{2}+\|f\|_{L^{2}}^{2}+\varepsilon^{-1}\|u\|_{L^{2}}^{2}\|u\|_{H^{1}}^{2}+\|u\|_{L^{2}}\|u\|_{H^{1}}^{3}\right)
\end{aligned}
$$

where

$$
\beta=\min \left(\frac{c_{0}}{8}, \frac{\beta_{0}}{4 c_{0}^{*}}\right)
$$

is fixed once and for all. We observe that relation (5.4) holds true with $\alpha=\beta$. 
Multiplying (5.12) by $\exp (\beta s)$, integrating the result between 0 and $t$, we obtain, for $t \in\left[0, T_{0}\right]$

$$
\begin{aligned}
E(u(t), u(t))+\frac{\beta_{0}}{4} & \int_{0}^{t} \exp (\beta(s-t))\|u(s)\|_{H^{2}}^{2} d s \\
\leq & \exp (-\beta t) E(u(0), u(0))+\frac{C}{\beta}\left(1-e^{-\beta t}\right)\|f\|_{L^{\infty}\left(L^{2}\right)}^{2} \\
& +C \int_{0}^{t} \exp (\beta(s-t))\|u(s)\|_{H^{1}}^{2}\left(1+\varepsilon^{-1}\|u(s)\|_{L^{2}}^{2}\right) d s \\
& +C \int_{0}^{t} \exp (\beta(s-t))\|u(s)\|_{L^{2}}\|u(s)\|_{H^{1}}^{3} d s .
\end{aligned}
$$

We deduce from (5.13) and from the Korn inequality (2.2) that, for $t \in\left[0, T_{0}\right]$,

$$
\begin{aligned}
\sup _{s \in[0, t]}\|u(s)\|_{H^{1}}^{2} \leq & C\left[\|u(0)\|_{H^{1}}^{2}+\|f\|_{L^{\infty}\left(L^{2}\right)}^{2}\right. \\
& +\int_{0}^{t} \exp (\beta(s-t))\|u(s)\|_{H^{1}}^{2}\left(1+\varepsilon^{-1}\|u(s)\|_{L^{2}}^{2}\right) d s \\
& \left.\quad+\sup _{s \in[0, t]}\|u(s)\|_{H^{1}} \int_{0}^{t} \exp (\beta(s-t))\|u(s)\|_{L^{2}}\|u(s)\|_{H^{1}}^{2} d s\right] .
\end{aligned}
$$

This estimate together with (5.4) for $\alpha=\beta$ imply, for $t \in\left[0, T_{0}\right]$,

$$
\begin{array}{r}
\sup _{s \in[0, t]}\|u(s)\|_{H^{1}}^{2} \leq C\left[\|u(0)\|_{H^{1}}^{2}+\|f\|_{L^{\infty}\left(L^{2}\right)}^{2}+\varepsilon^{-1}\left\|u_{0}\right\|_{L^{2}}^{4}+\varepsilon^{-1}\left\|M_{h} f\right\|_{L^{\infty}\left(L^{2}\right)}^{4}+\varepsilon^{3}\|f\|_{L^{\infty}\left(L^{2}\right)}^{4}\right. \\
\left.+\sup _{s \in[0, t]}\|u(s)\|_{H^{1}}\left(\|u(0)\|_{L^{2}}^{3}+\left\|M_{h} f\right\|_{L^{\infty}\left(L^{2}\right)}^{3}+\varepsilon^{3}\|f\|_{L^{\infty}\left(L^{2}\right)}^{3}\right)\right]
\end{array}
$$

Finally, using the Young inequality, we get, for $0<\varepsilon \leq \varepsilon_{0}$ and $t \in\left[0, T_{0}\right]$,

$$
\begin{aligned}
& \sup _{[0, t]}\|u(t)\|_{H^{1}}^{2} \leq C_{1}\left[\|u(0)\|_{H^{1}}^{2}+\|u(0)\|_{L^{2}}^{6}+\|f\|_{L^{\infty}\left(L^{2}\right)}^{2}+\varepsilon^{6}\|f\|_{L^{\infty}\left(L^{2}\right)}^{6}\right. \\
& \left.\quad+\varepsilon^{-1}\left\|u_{0}\right\|_{L^{2}}^{4}+\varepsilon^{-1}\left\|M_{h} f\right\|_{L^{\infty}\left(L^{2}\right)}^{4}+\left\|M_{h} f\right\|_{L^{\infty}\left(L^{2}\right)}^{6}+\varepsilon^{3}\|f\|_{L^{\infty}\left(L^{2}\right)}^{4}\right] \stackrel{\text { def }}{=} R_{0}^{2}(\varepsilon),
\end{aligned}
$$

for some constant $C_{1}>1$ independent of $\varepsilon$. In particular, one has that $\left\|u\left(T_{0}\right)\right\|_{H^{1}} \leq R_{0}$. It is now clear that if, for $0<\varepsilon \leq \varepsilon_{0}, R_{0}(\varepsilon)$ is such that

$$
\frac{\beta_{0}}{2}>C_{0} \varepsilon^{1 / 2} R_{0}(\varepsilon)+C_{0} \varepsilon R_{0}(\varepsilon)^{2}+C_{0} \varepsilon^{2}
$$

then this contradicts the statement (5.11). On the other hand, we remark that, due to Proposition 3.6, there exists a positive constant $C$ such that

$$
\|u(0)\|_{L^{2}}^{2} \leq\left\|v_{h}(0)\right\|_{L^{2}}^{2}+C \varepsilon^{2}\|u(0)\|_{H^{1}}^{2} .
$$


The definition of $R_{0}$ given in (5.14) as well as the property (5.16) show that, under the hypothesis (5.1), $R_{0}(\varepsilon)^{2}$ can be bounded as follows, for $0<\varepsilon \leq \varepsilon_{0}$,

$$
\begin{aligned}
R_{0}(\varepsilon)^{2} \leq & C_{2}\left(\varepsilon^{-1}\left(\kappa_{1}^{2}+\kappa_{0}^{4}+K_{1}^{2}+K_{0}^{4}\right)+\kappa_{0}^{6}+K_{0}^{6}+\varepsilon K_{1}^{4}+\varepsilon^{3}\left(\kappa_{1}^{4}+K_{1}^{6}\right)+\varepsilon^{6} \kappa_{1}^{6}\right) \\
& \stackrel{\text { def }}{=} R_{1}^{2}(\varepsilon),
\end{aligned}
$$

for some positive constant $C_{2}$ independent of $\varepsilon$. One notices that one can choose the positive numbers $\kappa_{0}, \kappa_{1}, K_{0}, K_{1}$ small enough (and independent of $\varepsilon$ ) so that, for $0<\varepsilon \leq \varepsilon_{0}$,

$$
\frac{\beta_{0}}{2}>C_{0} \varepsilon^{1 / 2} R_{1}(\varepsilon)+C_{0} \varepsilon R_{1}(\varepsilon)^{2}+C_{0} \varepsilon^{2} .
$$

For such a choice of constants, the assumption (5.1) implies the inequalities (5.10) and (5.15). We have thus proved that, under the conditions (5.1), the Navier-Stokes equations (1.2) admit a unique globally defined strong solution $u(t) \in C^{0}\left([0,+\infty), V_{\varepsilon}\right)$.

In what follows, we assume that $\varepsilon_{0}, \kappa_{0}, \kappa_{1}, K_{0}$ and $K_{1}$ satisfy the condition (5.18), for $0<\varepsilon \leq \varepsilon_{0}$. Now that we have proved the global existence of strong solutions of the NavierStokes equations (1.2), we want to estimate the size of $\|u(t)\|_{H^{1}}$ when $t$ is going to infinity. We choose data satisfying the conditions (5.1) and we set $r_{0}^{2}=\left\|v_{h}(0)\right\|_{L^{2}}^{2}+\varepsilon^{2} C\|u(0)\|_{H^{1}}^{2}$. From (5.4), we at once deduce that there exists a time $\tau_{0} \equiv \tau_{0}\left(r_{0}\right)$, depending on $r_{0}$, such that, for $t \geq \tau_{0}$, we have,

$$
\|u(t)\|_{L^{2}}^{2}+\frac{c_{0}}{2} \int_{0}^{t}(\exp \beta(s-t))\|u(s)\|_{H^{1}}^{2} d s \leq C \mathcal{R}^{2},
$$

where

$$
\mathcal{R}^{2}=\left\|M_{h} f\right\|_{L^{\infty}\left(L^{2}\right)}^{2}+\varepsilon^{2}\left\|\left(I-M_{h}\right) f\right\|_{L^{\infty}\left(L^{2}\right)}^{2} .
$$

Multiplying the inequality (5.12) by $\exp \beta t$ and integrating from $T$ to $t$, where $\tau_{0} \leq T \leq t$, we obtain

$$
\begin{aligned}
E(u(t), u(t)) \leq & \exp (-\beta(t-T)) E(u(T), u(T))+C \beta^{-1}\left(1-e^{-\beta(t-T)}\right)\|f\|_{L^{\infty}\left(L^{2}\right)}^{2} \\
& +C \int_{T}^{t}(\exp \beta(s-t))\|u(s)\|_{H^{1}}^{2}\left(1+\|u(s)\|_{L^{2}}\|u(s)\|_{H^{1}}\right) d s \\
& +\varepsilon^{-1} C \int_{T}^{t}(\exp \beta(s-t))\|u(s)\|_{L^{2}}^{2}\|u(s)\|_{H^{1}}^{2} d s .
\end{aligned}
$$

We set $T=\tau_{0}$. According to (5.20), there exists $\tau_{1} \geq \tau_{0}$ depending on $r_{1}$ where $r_{1}=\left\|u_{0}\right\|_{H^{1}}$ such that, for $t \geq \tau_{0}+\tau_{1}$,

$$
\begin{aligned}
\|u(t)\|_{H^{1}}^{2} \leq & C\|f\|_{L^{\infty}\left(L^{2}\right)}^{2}+\varepsilon^{-1} C \int_{\tau_{0}}^{t}(\exp \beta(s-t))\|u(s)\|_{L^{2}}^{2}\|u(s)\|_{H^{1}}^{2} d s \\
& +C \int_{\tau_{0}}^{t}(\exp \beta(s-t))\|u(s)\|_{H^{1}}^{2}\left(1+\|u(s)\|_{L^{2}}\|u(s)\|_{H^{1}}\right) d s .
\end{aligned}
$$

Due to (5.11) and (5.19), we deduce from (5.21), that, for $t \geq \tau_{0}+\tau_{1}$,

$$
\|u(t)\|_{H^{1}}^{2} \leq C\|f\|_{L^{\infty}\left(L^{2}\right)}^{2}+C \varepsilon^{-1} \mathcal{R}^{4}+C \mathcal{R}^{2}+C \mathcal{R}^{3} \varepsilon^{-1 / 2} \beta_{0} .
$$


We note that the term $\mathcal{R}^{3} \varepsilon^{-1 / 2} \beta_{0}$ can be written as

$$
\mathcal{R}^{3} \varepsilon^{-1 / 2} \beta_{0} \leq \mathcal{R}^{4} \varepsilon^{-1}+C \mathcal{R}^{2} .
$$

Finally, the above inequality as well as (5.22) imply that there exists a positive constant $C_{3}$ such that, for $0<\varepsilon \leq \varepsilon_{0}$, for $t \geq \tau_{0}+\tau_{1}$,

$$
\|u(t)\|_{H^{1}}^{2} \leq C_{3}\left(\|f\|_{L^{\infty}\left(L^{2}\right)}^{2}+\varepsilon^{-1} \mathcal{R}^{4}\right) .
$$

We thus have proved that there exist a bounded set $B_{\varepsilon}=B_{V_{\varepsilon}}(0, R)$, where $R^{2}=$ $C_{3}\left(\|f\|_{L^{\infty}\left(L^{2}\right)}^{2}+\varepsilon^{-1} \mathcal{R}^{4}\right)$ and a positive time $T_{1}$ such that, for $0<\varepsilon \leq \varepsilon_{0}$, for any initial data $u_{0}$ in $V_{\varepsilon}$, satisfying the conditions (5.1), the solution $u(t)$ of $(1.2)$ with $u(0)=u_{0}$ enters into the ball $B_{\varepsilon}$ at a time $t \leq T_{1}$ and stays there for any later time. We emphasize that the radius $R_{1}$ of this ball $B_{\varepsilon}$ depends only on $f$ and not of the size of the initial data $u_{0}$. In other terms, we have shown that $B_{\varepsilon}$ is an absorbing set for the solutions $u(t)$ whose initial data satisfy (5.1). In particular, if $f=0$, the solution $u(t)$ converges to zero when $t$ goes to infinity.

For later use in the next section, we also need the following theorem, which gives a more precise estimate of $\|u(t)\|_{L^{2}}$ and $\|u(t)\|_{H^{1}}$, when the size of the initial data and the forcing term is smaller.

Theorem 5.1. Let $\delta_{1}(\varepsilon)>0$ and $\delta_{2}(\varepsilon)>0$ be two functions which converge to 0 , when $\varepsilon$ goes to 0 and let $\kappa_{2}, \kappa_{3}, K_{2}, K_{3}$ be positive constants. There exists $\varepsilon_{1}>0$ small enough so that, for any $0<\varepsilon \leq \varepsilon_{1}$, if the initial data $u(0)$ and the forcing term $f=f_{\varepsilon}$ satisfy the following conditions,

$$
\begin{gathered}
\|u(0)\|_{H^{1}}^{2} \leq \kappa_{3}^{2} \delta_{1}(\varepsilon) \varepsilon^{-1}, \quad\left\|v_{h}(0)\right\|_{L^{2}}^{2} \leq \kappa_{2}^{2} \delta_{2}(\varepsilon) \\
\|f\|_{L^{\infty}\left(L^{2}\right)}^{2} \leq K_{3}^{2} \delta_{1}(\varepsilon) \varepsilon^{-1}, \quad\left\|M_{h} f\right\|_{L^{\infty}\left(L^{2}\right)}^{2} \leq K_{2}^{2} \delta_{2}(\varepsilon),
\end{gathered}
$$

then the strong solution $u(t)$ of (1.2) exists globally and the following estimates hold, for $0<\varepsilon \leq \varepsilon_{1}$ and $t \geq 0$,

$$
\begin{aligned}
\|u(t)\|_{L^{2}}^{2} & \leq K\left(\delta_{2}(\varepsilon)+\varepsilon \delta_{1}(\varepsilon)\right) \\
\int_{0}^{t}\|u(s)\|_{H^{1}}^{2} d s & \leq K(t+1)\left(\delta_{2}(\varepsilon)+\varepsilon \delta_{1}(\varepsilon)\right),
\end{aligned}
$$

and

$$
\begin{aligned}
\|u(t)\|_{H^{1}}^{2} & \leq K^{*}\left(\varepsilon^{-1} \delta_{1}(\varepsilon)+\varepsilon^{-1} \delta_{2}^{2}(\varepsilon)\right), \\
\int_{0}^{t}\|u(s)\|_{H^{2}}^{2} d s & \leq K^{*}(1+t)\left(\varepsilon^{-1} \delta_{1}(\varepsilon)+\varepsilon^{-1} \delta_{2}^{2}(\varepsilon)+\delta_{2}(\varepsilon)\right),
\end{aligned}
$$

where $K$ and $K^{*}$ are positive constants independent of $\varepsilon$.

Proof. Since $\delta_{1}(\varepsilon)$ and $\delta_{2}(\varepsilon)$ go to 0 when $\varepsilon$ converges to 0 , there exists $\varepsilon_{1}>0$ so that, for $0<\varepsilon \leq \varepsilon_{1}$, the conditions (5.24) imply the hypotheses (5.1) and (5.18), and thus the solution of (1.2) exists globally. The estimates (5.25) are a direct consequence of (5.3), (5.4) and (5.16). The first inequality in (5.26) is a direct consequence of (5.14), (5.16) and the hypotheses (5.24). Integrating the inequality (5.12) between 0 and $t$, arguing as above, but using the second estimate of (5.25) instead of the first one, we at once get the second estimate in (5.26). 
When $\delta_{2}(\varepsilon)$ is of order $\varepsilon$, which is the case when $v_{h}(0)$ is bounded in $L^{2}\left(\mathbb{T}^{2}\right)$ and when $M_{h} f$ converges to some function $f_{0}$ in $L^{2}\left(\mathbb{T}^{2}\right)$, we obtain the following improved inequality, as a consequence of Theorem 5.1.

Corollary 5.2. Assume that the hypotheses of Theorem 5.1 hold and that $\delta_{2}(\varepsilon)=O(\varepsilon)$. Then, for $0<\varepsilon \leq \varepsilon_{1}$, the strong solution $u(t)$ of (1.2) exists globally and the following estimates hold, for $t \geq 0$,

$$
\|u(t)\|_{L^{2}}^{2} \leq \widetilde{K} \varepsilon, \int_{0}^{t}\|u(s)\|_{H^{1}}^{2} d s \leq \widetilde{K} \varepsilon(t+1),
$$

and

$$
\int_{0}^{t}\left\|\frac{d u}{d t}(s)\right\|_{L^{2}}^{2} d s \leq \widetilde{K}^{*}(t+1)\left(\varepsilon^{-1} \delta_{1}(\varepsilon)+\varepsilon\right)
$$

where $\widetilde{K}$ and $\widetilde{K}^{*}$ are positive constants independent of $\varepsilon$.

Proof. The estimates (5.27) are an immediate consequence of (5.25).

The proof of the inequality (5.28) is standard. We reproduce it here since we want to make precise the dependency on $\varepsilon$. Taking the inner product in $H_{\varepsilon}$ of the equations (1.2) with $\frac{d u}{d t}$, using the Green formula (2.17), we obtain, for $t \geq 0$,

$$
\frac{3}{4}\left\|\frac{d u}{d t}(t)\right\|_{L^{2}}^{2}+\frac{1}{4} \frac{d}{d t} E(u(t), u(t)) \leq\|f(t)\|_{L^{2}}^{2}+\left|\int_{\Omega_{\varepsilon}} u \nabla u \frac{d u}{d t} d x\right| .
$$

In order to estimate the last term in the right-hand side of the above inequality, we write $u$ as $u=M_{h} u+\left(I-M_{h}\right) u \equiv v_{h}+w^{*}$, which gives

$$
\int_{\Omega_{\varepsilon}} u \nabla u \frac{d u}{d t} d x=\int_{\Omega_{\varepsilon}} w^{*} \nabla u \frac{d u}{d t} d x+\int_{\Omega_{\varepsilon}} v_{h} \nabla v_{h} \frac{d v_{h}}{d t} d x+\int_{\Omega_{\varepsilon}} v_{h} \nabla w^{*} \frac{d u}{d t} d x .
$$

Using the above equality as well as the estimates (3.27), (3.28), (3.32), (3.37) we obtain

$$
\begin{gathered}
\left|\int_{\Omega_{\varepsilon}} u \nabla u \frac{d u}{d t} d x\right| \leq\left\|w^{*}\right\|_{L^{\infty}}\|u\|_{H^{1}}\left\|\frac{d u}{d t}\right\|_{L^{2}}+\left\|v_{h}\right\|_{L^{4}}\left\|\nabla w^{*}\right\|_{L^{4}}\left\|\frac{d u}{d t}\right\|_{L^{2}} \\
+C\left\|v_{h}\right\|_{L^{4}}\left\|\nabla_{h} v_{h}\right\|_{L^{4}}\left\|\frac{d v_{h}}{d t}\right\|_{L^{2}}
\end{gathered}
$$

and also

$$
\begin{gathered}
\left|\int_{\Omega_{\varepsilon}} u \nabla u \frac{d u}{d t} d x\right| \leq C \varepsilon^{1 / 2}\left\|w^{*}\right\|_{H^{2}}\|u\|_{H^{1}}\left\|\frac{d u}{d t}\right\|_{L^{2}}+C\left\|v_{h}\right\|_{L^{2}}^{1 / 2}\left\|v_{h}\right\|_{H^{1}}^{1 / 2}\left\|w^{*}\right\|_{H^{2}}\left\|\frac{d u}{d t}\right\|_{L^{2}} \\
+C \varepsilon^{-1 / 2}\left\|v_{h}\right\|_{L^{2}}^{1 / 2}\left\|v_{h}\right\|_{H^{1}}\left\|v_{h}\right\|_{H^{2}}^{1 / 2}\left\|\frac{d v_{h}}{d t}\right\|_{L^{2}} .
\end{gathered}
$$

The estimates (5.29) and (5.30) imply, for $t \geq 0$,

$$
\begin{aligned}
\frac{1}{2}\left\|\frac{d u}{d t}(t)\right\|_{L^{2}}^{2}+\frac{1}{4} \frac{d}{d t} E(u(t), u(t)) \leq & \|f(t)\|_{L^{2}}^{2}+C \varepsilon\|u\|_{H^{2}}^{2}\|u\|_{H^{1}}^{2}+C\left\|v_{h}\right\|_{L^{2}}\left\|v_{h}\right\|_{H^{1}}\|u\|_{H^{2}}^{2} \\
& +C \varepsilon^{-1}\left\|v_{h}\right\|_{L^{2}}\left\|v_{h}\right\|_{H^{1}}^{2}\left\|v_{h}\right\|_{H^{2}} .
\end{aligned}
$$


Integrating the inequality (5.31) from 0 to $t$, applying the inequality (2.2) and taking into account the estimates satisfied by the initial data and the forcing term, we get, for any $t \geq 0$

$$
\begin{aligned}
\int_{0}^{t}\left\|\frac{d u}{d t}(s)\right\|_{L^{2}}^{2} d s+\frac{c_{0}}{2}\|u(t)\|_{H^{1}}^{2} \leq & \left(c_{0}^{*} \kappa_{3}^{2}+2 t K_{3}^{2}\right) \delta_{1}(\varepsilon) \varepsilon^{-1}+C \varepsilon \int_{0}^{t}\|u(s)\|_{H^{2}}^{2}\|u(s)\|_{H^{1}}^{2} d s \\
& +C \int_{0}^{t}\left\|v_{h}(s)\right\|_{L^{2}}\left\|v_{h}(s)\right\|_{H^{1}}\|u(s)\|_{H^{2}}^{2} d s \\
& +C \varepsilon^{-1} \int_{0}^{t}\left\|v_{h}(s)\right\|_{L^{2}}\left\|v_{h}(s)\right\|_{H^{1}}^{2}\left\|v_{h}(s)\right\|_{H^{2}} d s .
\end{aligned}
$$

Remarking that

$$
\int_{0}^{t}\left\|v_{h}(s)\right\|_{L^{2}}\left\|v_{h}(s)\right\|_{H^{1}}^{2}\left\|v_{h}(s)\right\|_{H^{2}} d s \leq \int_{0}^{t}\left(\left\|v_{h}(s)\right\|_{L^{2}}^{2}\left\|v_{h}(s)\right\|_{H^{2}}^{2}+\left\|v_{h}(s)\right\|_{H^{1}}^{4}\right) d s .
$$

and using the estimates (5.26) and (5.27), we deduce finally from (5.32) that, for $t \geq 0$,

$$
\int_{0}^{t}\left\|\frac{d u}{d t}(s)\right\|_{L^{2}}^{2} d s+\frac{c_{0}}{2}\|u(t)\|_{H^{1}}^{2} \leq \widetilde{K}^{*}(1+t)\left(\varepsilon^{-1} \delta_{1}(\varepsilon)+\varepsilon\right),
$$

which proves the estimate (5.28).

\section{The LiMit PROBLEM}

In order to simplify the notations, we assume in the whole section, without loss of generality, that $\nu=1$. Also, we will identify in the classical way the hyperplane $y_{3}=0 \mathrm{in}$ $\mathbb{R}^{3}$ with $\mathbb{R}^{2}$; that is, if $v^{*}$ is the three-components vector $\left(v_{1}^{*}, v_{2}^{*}, 0\right)$, we still denote by the same letter $v^{*}$ the two-components vector $\left(v_{1}^{*}, v_{2}^{*}\right)$.

As we indicated in the Introduction, the forcing term $f \equiv f_{\varepsilon}$, in the Navier-Stokes equations (1.2), can depend on $\varepsilon$. So the following natural question arises: when $M_{h} f$ converges to a two-dimensional forcing term $f_{0}\left(x_{1}, x_{2}\right)$, do the solutions of the equations (1.2) converge to those of a limit system defined on $\mathbb{T}^{2}$. The aim of this section is to prove that this is actually true. We begin by determining the limit equations and by introducing the functional frame of this new equation.

6.1. Description of the limit equations. We begin with some remarks and additional notations. We recall that if $v$ is any smooth enough two-dimensional vector field defined on $\mathbb{T}^{2}$, its divergence is denoted by $\operatorname{div}_{\mathrm{h}} v=\partial_{1} v_{1}+\partial_{2} v_{2}$. Likewise, for any function $\varphi$ defined on $\mathbb{T}^{2}, \nabla_{h} \varphi$ is the two-dimensional vector $\left(\partial_{1} \varphi, \partial_{2} \varphi\right)$.

In order to define the limit equation of the divergence equation, we first recall (see Remark 3.1) that, if $U \in V_{\varepsilon}$, then

$$
\operatorname{div} M_{1} U=\frac{1}{g} \operatorname{div} g\left(M U_{1}, M U_{2}, 0\right) \equiv \frac{1}{g} \operatorname{div}_{\mathrm{h}} g\left(M U_{1}, M U_{2}\right)=0 .
$$

This property shows that, in the limit system, the usual condition of vanishing divergence shall be replaced by the vanishing of $\operatorname{div}_{\mathrm{h}}(g \cdot)$. 
Moreover, we notice that, if $\ell=\ell\left(x_{1}, x_{2}\right)$ is any $L^{1}\left(\mathbb{T}^{2}\right)$ function, depending only on $\left(x_{1}, x_{2}\right)$, then

$$
\varepsilon^{-1} \int_{\Omega_{\varepsilon}} \ell\left(x_{1}, x_{2}\right) d x=\int_{\mathbb{T}^{2}} g\left(x_{1}, x_{2}\right) \ell\left(x_{1}, x_{2}\right) d x_{1} d x_{2} .
$$

The above equality indicates that, when we consider the two-dimensional limit problem on $\mathbb{T}^{2}$, we should consider weighted Sobolev spaces with the weight $g$. For this reason, we denote by $L_{g}^{2}\left(\mathbb{T}^{2}\right)$ (respectively $H_{g}^{1}\left(\mathbb{T}^{2}\right)$ ) the space $L^{2}\left(\mathbb{T}^{2}\right)$ (respectively $H^{1}\left(\mathbb{T}^{2}\right)$ ) in the definition of which the measure $d x_{1} d x_{2}$ is replaced by $g\left(x_{1}, x_{2}\right) d x_{1} d x_{2}$. For the same reason, we introduce the following scalar products, defined, for any smooth enough two-dimensional vectors fields $\bar{v}$ and $\widetilde{v}$, by

$$
(\bar{v}, \widetilde{v})_{g}=\int_{\mathbb{T}^{2}} g \bar{v} \cdot \widetilde{v} d x_{1} d x_{2}, \quad E_{g}(\bar{v}, \widetilde{v})=\sum_{i, j=1}^{2}\left(\partial_{i} \bar{v}_{j}+\partial_{j} \bar{v}_{i}, \partial_{i} \widetilde{v}_{j}+\partial_{j} \widetilde{v}_{i}\right)_{g} .
$$

We further notice that if $f$ satisfies the condition $\left(\mathrm{H}_{f}\right)$, then the following corresponding condition holds for $M_{h} f$ :

$\left(\mathrm{H}_{f}^{g}\right) \quad$ for all $t \geq 0, \quad a_{1} \int_{\mathbb{T}^{2}} g\left(x_{h}\right)\left(M f_{1}\right)\left(t, x_{h}\right) d x_{h}+a_{2} \int_{\mathbb{T}^{2}} g\left(x_{h}\right)\left(M f_{2}\right)\left(t, x_{h}\right) d x_{h}=0$, for all constants $a_{1}, a_{2}$ such that $a_{1} \partial_{1} g+a_{2} \partial_{2} g \equiv 0$.

Moreover, if $M_{h} f_{\varepsilon}$ converges in $L^{\infty}\left((0, \infty), L^{2}\left(\mathbb{T}^{2}\right)^{2}\right)$ to a two-dimensional forcing term $f_{0}$ where $f_{0}$ belongs to $L^{\infty}\left((0, \infty) ; L_{g}^{2}\left(\mathbb{T}^{2}\right)^{2}\right.$ and if $f$ satisfies the condition $\left(\mathrm{H}_{f}\right)$, then $f_{0}$ satisfies the condition $\left(\mathrm{H}_{f}^{g}\right)$.

In the same way, if $u_{0}$ satisfies the condition $(\mathrm{H})$, then $M_{h} u_{0}$ fulfills the corresponding condition

$$
\begin{aligned}
a_{1} \int_{\mathbb{T}^{2}} g\left(x_{h}\right)\left(M u_{0}\right)_{1}\left(x_{h}\right) d x_{h}+a_{2} \int_{\mathbb{T}^{2}} g\left(x_{h}\right)\left(M u_{0}\right)_{2}\left(x_{h}\right) d x_{h}=0, \\
\text { for all constants } a_{1}, a_{2} \text { such that } a_{1} \partial_{1} g+a_{2} \partial_{2} g \equiv 0 .
\end{aligned}
$$

The above discussion shows that the spaces corresponding to $V_{\varepsilon}$ and $H_{\varepsilon}$ in the limit case are the following ones:

$V_{0}=\left\{v \in H_{g}^{1}\left(\mathbb{T}^{2}\right)^{2} \mid v\right.$ is periodic in $\left(x_{1}, x_{2}\right), \operatorname{div}_{\mathrm{h}} g v=0, v$ satisfies the condition $\left.\left(\mathrm{H}^{g}\right)\right\}$. Below, we shall also use the following space $H_{0}=\left\{v \in L_{g}^{2}\left(\mathbb{T}^{2}\right)^{2} \mid v\right.$ is periodic in $\left(x_{1}, x_{2}\right), \operatorname{div}_{\mathrm{h}} g v=0, v$ satisfies the condition $\left.\left(\mathrm{H}^{g}\right)\right\}$.

From now on, we assume that $M_{h} f_{\varepsilon}$ converges to the two-dimensional vector field $f_{0}$ in $L^{\infty}\left((0, \infty) ; L^{2}\left(\mathbb{T}^{2}\right)^{2}\right)$ and that

$$
\left\|M_{h}\left(f_{\varepsilon}\right)-f_{0}\right\|_{L^{\infty}\left(L^{2}\left(\mathbb{T}^{2}\right)\right)}^{2} \leq \delta_{3}(\varepsilon),
$$

where $\delta_{3}(\varepsilon)$ is a positive function of $\varepsilon$ which goes to 0 when $\varepsilon$ goes to 0 . 
In order to determine easily the limit equation, we turn to the variational form of the equations (1.2), which consists in finding $u \in C^{0}\left([0, T) ; V_{\varepsilon}\right)$, where $T>0$, such that,

$$
\left(\partial_{t} u, u^{*}\right)+\frac{1}{2} E\left(u, u^{*}\right)+\left(u \cdot \nabla u, u^{*}\right)=\left(f_{\varepsilon}, u^{*}\right) \text {, for all } u^{*} \in L^{2}\left((0, T) ; V_{\varepsilon}\right), u(0)=u_{0} \text {. }
$$

Let $v^{*}$ be any (smooth enough) two-dimensional vector field in $V_{0}$ independent of $\varepsilon$. If we replace $u^{*}$ in the above equality by $M_{1} v^{*} \equiv\left(v^{*}, \frac{x_{3}}{g} v^{*} \cdot \nabla g\right)$ (see (3.2)), use the boundary conditions, let $\varepsilon$ go to zero and make the Ansatz that the limit equations do not depend on $x_{3}$, we obtain the limit variational problem, which consists in finding $v^{0} \in C^{0}\left([0, T) ; V_{0}\right)$ such that,

$$
\begin{aligned}
& \left(\partial_{t} v^{0}, v^{*}\right)_{g}+\frac{1}{2} E_{g}\left(v^{0}, v^{*}\right)+2\left(g^{-1}\left(v_{1}^{0} \partial_{1} g+v_{2}^{0} \partial_{2} g\right), g^{-1}\left(v_{1}^{*} \partial_{1} g+v_{2}^{*} \partial_{2} g\right)\right)_{g} \\
& +\left(v^{0} \cdot \nabla_{h} v^{0}, v^{*}\right)_{g}=\left(f_{0}, v^{*}\right)_{g}, \quad \text { for all } v^{*} \in L^{2}\left((0, T) ; V_{0}\right), \quad v^{0}(0)=M_{h} u(0) .
\end{aligned}
$$

We shall show below that the solution $v^{0}(t)$ of the equation (6.3) is indeed the limit of $M_{h} u(t)$, when $\varepsilon$ goes to zero.

Using the fact that $g v^{0}$ and $g v^{*}$ are divergence-free and making a few calculations analog to the ones made in (2.17), we see that the above variational equation leads to the following Navier-Stokes type equations. Find $v^{0} \in C^{0}\left([0, T), V_{0}\right)$ satisfying the equations

$$
\begin{aligned}
& \partial_{t} v^{0}-\frac{1}{g} \sum_{i=1}^{2} \partial_{i}\left(g \partial_{i} v^{0}\right)+\frac{1}{g^{2}} \sum_{i=1}^{2} v_{i}^{0} \partial_{i}\left(g \nabla_{h} g\right)+v^{0} \cdot \nabla_{h} v^{0}=f_{0}-\nabla_{h} p, \\
& \operatorname{div}_{\mathrm{h}} g v^{0}=0, \\
& \left.v^{0}\right|_{t=0}=v_{0}^{0} .
\end{aligned}
$$

We now introduce the projection $\mathbb{P}_{g}: L_{g}^{2}\left(\mathbb{T}^{2}\right)^{2} \rightarrow L_{g}^{2}\left(\mathbb{T}^{2}\right)^{2}$, which is the orthogonal projection in $L_{g}^{2}\left(\mathbb{T}^{2}\right)^{2}$ onto the subspace of vector fields $v$ satisfying $\operatorname{div}_{\mathrm{h}} g v=0$ (notice that this space is the orthogonal space in $L_{g}^{2}\left(\mathbb{T}^{2}\right)^{2}$ of the set of all two-dimensional gradient vectors). Applying this Leray projector in the above equation, we obtain the following reduced Navier-Stokes type equations:

$$
\begin{aligned}
& \partial_{t} v^{0}-\mathbb{P}_{g}\left(\frac{1}{g} \sum_{i=1}^{2} \partial_{i}\left(g \partial_{i} v^{0}\right)\right)+\mathbb{P}_{g}\left(\frac{1}{g^{2}} \sum_{i=1}^{2} v_{i}^{0} \partial_{i}\left(g \nabla_{h} g\right)\right)+\mathbb{P}_{g}\left(v^{0} \cdot \nabla_{h} v^{0}\right)=\mathbb{P}_{g} f_{0}, \\
& \operatorname{div}_{\mathrm{h}} g v^{0}=0 \\
& \left.v^{0}\right|_{t=0}=v_{0}^{0} .
\end{aligned}
$$

Before comparing the solutions of the Navier-Stokes equations (1.2)-(1.3) with those of the limit equations (6.4) or (6.5), we will do some comments of the 2D Navier-Stokes type equations (6.4). These equations have the same properties as the classical 2D Navier-Stokes equations. First, we notice that the mapping

$$
\left(v^{*}, v^{* *}\right) \in V_{0}^{2} \mapsto a_{g}\left(v^{*}, v^{* *}\right)=\frac{1}{2} E_{g}\left(v^{*}, v^{* *}\right)+2\left(g^{-1}\left(v_{1}^{*} \partial_{1} g+v_{2}^{*} \partial_{2} g\right), g^{-1}\left(v_{1}^{* *} \partial_{1} g+v_{2}^{* *} \partial_{2} g\right)\right)_{g}
$$


defines a self-adjoint bilinear form on $V_{0}$. Because of the condition $\left(\mathrm{H}^{g}\right)$, the quadratic form $a_{g}\left(v^{*}, v^{*}\right)$ is positive definite and there exists a positive constant $c_{4}$, such that

$$
a_{g}\left(v^{*}, v^{*}\right) \geq c_{4}\left\|v^{*}\right\|_{H_{g}^{1}}^{2},
$$

where

$$
\left\|v^{*}\right\|_{H_{g}^{1}}^{2}=\left\|v^{*}\right\|_{L_{g}^{2}}^{2}+\left\|\nabla_{h} v^{*}\right\|_{L_{g}^{2}}^{2}
$$

The inequality (6.6) can either be proved directly, or as a direct consequence of (2.2). Indeed, with every $v^{*} \in V_{0}$, one associates the vector $M_{1} v^{*}=\left(v_{1}^{*}, v_{2}^{*}, \frac{x_{3}}{g} v^{*} \cdot \nabla g\right)$, which belongs to the space $\mathcal{H}^{1}$. We remark that

$$
\varepsilon a_{g}\left(v^{*}, v^{*}\right)=\frac{1}{2} E\left(M_{1} v^{*}, M_{1} v^{*}\right)-\sum_{i=1}^{2} \int_{\Omega_{\varepsilon}}\left(x_{3} \partial_{i}\left(\frac{1}{g} v^{*} \cdot \nabla g\right)\right)^{2} d x .
$$

Applying thus the inequality (2.2) to $M_{1} v^{*}$, taking into account that the second term in the right hand side of (6.7) is of order $\varepsilon^{3}$ and choosing $\varepsilon$ small enough, we get

$$
\varepsilon a_{g}\left(v^{*}, v^{*}\right) \geq \frac{1}{2} E\left(M_{1} v^{*}, M_{1} v^{*}\right)-\varepsilon^{3} C_{2}^{*}\left\|v^{*}\right\|_{H_{g}^{1}}^{2} \geq C_{3}^{*} \varepsilon\left\|v^{*}\right\|_{H_{g}^{1}}^{2}\left(1-C_{4}^{*} \varepsilon^{2}\right),
$$

which at once implies (6.6), for $\varepsilon>0$ small enough.

Since the bilinear form $a_{g}(\cdot, \cdot)$ is a coercive continuous symmetric bilinear form on the space $V_{0}$, we can claim as in Section 2.2 that there exists a (sectorial) self-adjoint positive operator $A_{0}$ on $H_{0}$ with domain $D\left(A_{0}\right)=\left\{v \in V_{0} \mid A_{0} v \in H_{0}\right\}$ such that, for all $v \in D\left(A_{0}\right)$

$$
\left(A_{0} v, v^{*}\right)_{g}=a_{g}\left(v, v^{*}\right), \quad \text { for all } v^{*} \in V_{0} \text {. }
$$

One can argue as in the classical regularity proofs to show that $D\left(A_{0}\right)=V_{0} \cap H^{2}\left(\mathbb{T}^{2}\right)^{2}$. It follows again as in the classical case that the operator $A_{0}$ is equal to

$$
A_{0} v^{0}=-\mathbb{P}_{g}\left(\frac{1}{g} \sum_{i=1}^{2} \partial_{i}\left(g \partial_{i} v^{0}\right)\right)+\mathbb{P}_{g}\left(\frac{1}{g^{2}} \sum_{i=1}^{2} v_{i}^{0} \partial_{i}\left(g \nabla_{h} g\right)\right) .
$$

Let $f_{0}$ be a forcing term in $L^{\infty}\left((0,+\infty), L_{g}^{2}\left(\mathbb{T}^{2}\right)^{2}\right)$ satisfying the condition $\left(\mathrm{H}_{f}^{g}\right)$. Again as in the case of the classical 2D Navier-Stokes equations, one can show, by using a Galerkin method or the Fujita-Kato method (see [9]) that, for any $v^{0}(0) \in H_{0}$, the equation (6.4) has a strong solution $v_{0}(t)$ in $C^{0}\left([0, \tau), H_{0}\right) \cap L_{l o c}^{2}\left((0, \tau), V_{0} \cap H^{2}\left(\mathbb{T}^{2}\right)^{2}\right) \cap H_{l o c}^{1}\left((0, \tau), H_{0}\right)$, for a positive time $\tau$. If $v_{0}(0)$ is in $V_{0}$, then the solution $v_{0}(t)$ belongs also to $C^{0}\left([0, \tau), V_{0}\right)$. The proof of the uniqueness of this classical solution in the class of the weak solutions follows the lines of the proof for the two-dimensional Navier-Stokes equations. Actually this unique solution is global in time (that is $\tau=+\infty$ ). As in the classical case, one also shows that the solutions are locally Lipschitzian functions of the initial data. More precisely, for any $v_{0}^{0}$ and $v_{0}^{1}$ in $H_{0}$ (respectively in $V_{0}$ ), and for any time $T>0$, if $v^{0}(t)$ and $v^{1}(t)$ denote the solutions of the equations (6.5) with initial data $v_{0}^{0}$ and $v_{0}^{1}$, we have, for $0 \leq t \leq T$,

$$
\left\|v^{0}(t)-v^{1}(t)\right\|_{L_{g}^{2}} \leq C\left(T,\left\|v_{0}^{0}\right\|_{L_{g}^{2}},\left\|v_{0}^{1}\right\|_{L_{g}^{2}}\right)\left\|v_{0}^{0}-v_{0}^{1}\right\|_{L_{g}^{2}}
$$

(respectively,

$$
\left.\left\|v^{0}(t)-v^{1}(t)\right\|_{H_{g}^{1}} \leq \widetilde{C}\left(T,\left\|v_{0}^{0}\right\|_{H_{g}^{1}},\left\|v_{0}^{1}\right\|_{H_{g}^{1}}\right)\left\|v_{0}^{0}-v_{0}^{1}\right\|_{H_{g}^{1}}\right),
$$


where $C\left(T,\left\|v_{0}^{0}\right\|_{L_{g}^{2}},\left\|v_{0}^{1}\right\|_{L_{g}^{2}}\right)$ and $\widetilde{C}\left(T,\left\|v_{0}^{0}\right\|_{H_{g}^{1}},\left\|v_{0}^{1}\right\|_{H_{g}^{1}}\right)$ are positive functions (of exponential type) of the size of the initial data and on $T$.

Next we quickly recall how to obtain the bounds of the $L_{g}^{2}$ and $H_{g}^{1}$-norms of the solution $v^{0}(t)$ of the equations (6.5) and how to show that the $H_{g}^{1}$ norm of $v^{0}(t)$ depends only of the size of the forcing term $f_{0}$ if $t$ is large enough. Since the proof is classical, we let the details to the reader. Replacing $v^{*}$ by $v^{0}(t)$ in (6.3), applying the Korn inequality (6.6) and remarking that $\left(v^{0} \cdot \nabla_{h} v^{0}, v^{0}\right)_{g}=0$, we show that there exists a positive number $\alpha_{0}$ such that, for $0<\alpha \leq \alpha_{0}$,

$$
\frac{\mathrm{d}}{\mathrm{d} t}\left\|v^{0}\right\|_{L_{g}^{2}}^{2}+\alpha\left\|v^{0}\right\|_{L_{g}^{2}}^{2}+\frac{c_{4}}{2}\left\|v^{0}\right\|_{H_{g}^{1}}^{2} \leq C\left\|A_{0}^{-1 / 2} f_{0}\right\|_{L_{g}^{2}}^{2} .
$$

Multiplying (6.9) by $\exp (\alpha s)$, integrating the result between 0 and $t$, we get, for $t>0$,

$$
\begin{aligned}
\left\|v^{0}(t)\right\|_{L_{g}^{2}}^{2}+\frac{c_{4}}{2} \exp (-\alpha t) \int_{0}^{t}(\exp \alpha s)\left\|v^{0}(s)\right\|_{H_{g}^{1}}^{2} d s & \leq \\
& \exp (-\alpha t)\left\|v^{0}(0)\right\|_{L_{g}^{2}}^{2}+\frac{C}{\alpha}\left(1-e^{-\alpha t}\right)\left\|A_{0}^{-1 / 2} f_{0}\right\|_{L^{\infty}\left(L_{g}^{2}\right)}^{2} .
\end{aligned}
$$

Integrating the inequality (6.9) between $t-\theta>0$ and $t$, we get, by using (6.10),

$$
\int_{t-\theta}^{t}\left\|v_{0}(s)\right\|_{H_{g}^{1}}^{2} d s \leq \frac{2}{c_{4}}\left(\exp \left(-\alpha_{0}(t-\theta)\right)\left\|v^{0}(0)\right\|_{L_{g}^{2}}^{2}+C\left(\theta+\alpha_{0}^{-1}\right)\left\|A_{0}^{-1 / 2} f_{0}\right\|_{L^{\infty}\left(L_{g}^{2}\right)}^{2} .\right.
$$

Next replacing $v^{*}$ by $A_{0} v^{0}(t)$ in (6.3) and using the appropriate classical Sobolev inequalities in two-dimensions, we obtain that, for $t>0$,

$$
\begin{aligned}
\frac{1}{2} \frac{\mathrm{d}}{\mathrm{d} t} a_{g}\left(v^{0}(t), v^{0}(t)\right)+ & \left\|A_{0} v^{0}(t)\right\|_{L_{g}^{2}}^{2} \leq \\
& \left\|M_{h} f_{0}(t)\right\|_{L_{g}^{2}}\left\|A_{0} v^{0}(t)\right\|_{L_{g}^{2}}+C\left\|v^{0}(t)\right\|_{L_{g}^{2}}^{\frac{1}{2}}\left\|v^{0}(t)\right\|_{H_{g}^{1}}\left\|A_{0} v^{0}\right\|_{L_{g}^{2}}^{\frac{3}{2}},
\end{aligned}
$$

which implies, by the Young inequality, that

$$
\frac{\mathrm{d}}{\mathrm{d} t} a_{g}\left(v^{0}(t), v^{0}(t)\right)+\left\|A_{0} v^{0}(t)\right\|_{L_{g}^{2}}^{2} \leq 2\left\|M_{h} f_{0}(t)\right\|_{L_{g}^{2}}^{2}+C\left\|v^{0}(t)\right\|_{L_{g}^{2}}^{2}\left\|v^{0}(t)\right\|_{H_{g}^{1}}^{4} .
$$

Integrating the inequality (6.12), applying the Gronwall lemma and using the Korn inequality (6.6) as well as the inequalities (6.10), (6.11), we get for $0 \leq t \leq 1$,

$$
\begin{aligned}
\left\|v^{0}(t)\right\|_{H_{g}^{1}}^{2} \leq c_{4}^{-1}\left(c_{0}^{*}\left\|v^{0}(0)\right\|_{H_{g}^{1}}^{2}+2\left\|f_{0}\right\|_{L^{\infty}\left(L_{g}^{2}\right)}^{2}\right) & \\
& \quad \exp \frac{2 C}{c_{4}}\left(\left\|v^{0}(0)\right\|_{L_{g}^{2}}^{2}+C\left(1+\alpha_{0}^{-1}\right)\left\|A_{0}^{-1 / 2} f_{0}\right\|_{L^{\infty}\left(L_{g}^{2}\right)}^{2}\right)^{2} .
\end{aligned}
$$

In order to obtain a uniform bound with respect to $t$, when $t \geq 1$, we proceed in the following way. We integrate the inequality (6.12), apply the uniform Gronwall lemma as well as the Korn inequality (6.6) and we obtain for $t \geq 0$,

$$
\begin{aligned}
&\left\|v^{0}(t)\right\|_{H_{g}^{1}}^{2} \leq c_{4}^{-1}\left(\frac{c_{0}^{*}}{t-\theta} \int_{\theta}^{t}\left\|v^{0}(s)\right\|_{H_{g}^{1}}^{2} d s\right.\left.+2(t-\theta)\left\|f_{0}\right\|_{L^{\infty}\left(L_{g}^{2}\right)}^{2}\right) \\
& \exp \left(C \int_{\theta}^{t}\left\|v^{0}(s)\right\|_{L_{g}^{2}}^{2}\left\|v^{0}(s)\right\|_{H_{g}^{1}}^{2} d s\right),
\end{aligned}
$$


where $\theta=\max (0, t-1)$ (for a statement of the uniform Gronwall inequality we refer to [7] or [43]). Using the estimates (6.10) and (6.11), we deduce from the above inequality for $0<t \leq \tau$,

$$
\begin{aligned}
\left\|v^{0}(t)\right\|_{H_{g}^{1}}^{2} \leq c_{4}^{-1}\left(\operatorname { e x p } \frac { 2 } { c _ { 4 } } \left[\exp \left(-\alpha_{0} \theta\right)\left\|v^{0}(0)\right\|_{L_{g}^{2}}^{2}\right.\right. & \left.\left.+C\left(1+\alpha_{0}^{-1}\right)\left\|A_{0}^{-1 / 2} f_{0}\right\|_{L^{\infty}\left(L_{g}^{2}\right)}^{2}\right]^{2}\right) \\
\times\left[2\left\|f_{0}\right\|_{L^{\infty}\left(L_{g}^{2}\right)}^{2}+C(1\right. & \left.+\frac{1}{\alpha_{0}(t-\theta)}\right)\left\|A_{0}^{-1 / 2} f_{0}\right\|_{L^{\infty}\left(L_{g}^{2}\right)}^{2} \\
& \left.+\frac{2 c_{0}^{*}}{c_{4}(t-\theta)} \exp \left(-\alpha_{0} \theta\right)\left\|v^{0}(0)\right\|_{L_{g}^{2}}^{2}\right],
\end{aligned}
$$

where $\theta=\max (0, t-1)$. The estimates (6.13) and (6.14) show that actually the solution $v^{0}(t)$ of the equations (6.5) is global. Moreover, if $t \geq t_{0}$, where $t_{0}>0$ depends only on $\left\|v^{0}(0)\right\|_{L_{g}^{2}}$, the following estimate holds,

$$
\begin{aligned}
\left\|v^{0}(t)\right\|_{H_{g}^{1}}^{2} \leq c_{4}^{-1}\left(\exp \frac{2}{c_{4}} C^{2}(1+\right. & \left.\left.\alpha_{0}^{-1}\right)^{2}\left\|A_{0}^{-1 / 2} f_{0}\right\|_{L^{\infty}\left(L_{g}^{2}\right)}^{4}\right) \\
& \times\left[2\left\|f_{0}\right\|_{L^{\infty}\left(L_{g}^{2}\right)}^{2}+C\left(1+\alpha_{0}^{-1}\right)\left\|A_{0}^{-1 / 2} f_{0}\right\|_{L^{\infty}\left(L_{g}^{2}\right)}^{2}\right] .
\end{aligned}
$$

Assume now that $f_{0}$ does not depend on the time $t$. In this case, the solution $v_{0}(t)$ of $(6.5)$ is classical and moreover $v_{0}(t)$ belongs to the space $C^{0}\left((0,+\infty), D\left(A_{0}\right)\right) \cap C^{1}\left((0,+\infty), H_{0}\right) \cap$ $L^{\infty}\left((0,+\infty), H_{0}\right)$. Also, we can introduce, for every $t \geq 0$, the mapping $S_{0}(t): v_{0}^{0} \in H_{0} \mapsto$ $S_{0}(t) v_{0}^{0} \equiv v^{0}(t) \in H_{0}$, where $v^{0}(t)$ is the solution of the Navier-Stokes equations (6.5). The family $S_{0}(t), t \geq 0$, defines a dynamical system on $H_{0}$ and also on $V_{0}$. Since $v^{0}(t)$ belongs to $C^{0}\left((0,+\infty), H^{2}\left(\mathbb{T}^{2}\right)^{2}\right)$, the mapping $S_{0}(t)$ is compact for $t>0$. Moreover, the estimates (6.15) show that there exists an absorbing set $\mathcal{B}_{0}$ in both spaces $H_{0}$ and $V_{0}$. Therefore, there exists a compact global attractor $\mathcal{A}_{0}$ that is, there exists a compact set $\mathcal{A}_{0}$, which is invariant under $S_{0}(t)$ (i.e. $S_{0}(t) \mathcal{A}_{0}=\mathcal{A}_{0}$ ), and which attracts every bounded set of $H_{0}$ in the topology of $V_{0}$, that is, for every bounded set $B \in H_{0}$ and for every positive number $\eta$, there exists a positive time $\tau=\tau(B, \eta)>0$ such that, for $t \geq \tau$,

$$
S_{0}(t) B \subset N_{V_{0}}\left(\mathcal{A}_{0}, \eta\right),
$$

where $N_{V_{0}}\left(\mathcal{A}_{0}, \eta\right)$ is the $\eta$-neighbourhood of $\mathcal{A}_{0}$ in $V_{0}$. In Section 7 , we shall compare the compact global attractor $\mathcal{A}_{0}$ of the limit equations (6.5) with the compact attractor $\mathcal{A}_{\varepsilon}$ of the equations (1.2), for $\varepsilon>0$ small enough.

6.2. Convergence properties. We next compare the orbits of the Navier-Stokes equations (1.2) with those of the limit equations (6.5) on finite time intervals $(0, T]$, under the conditions (5.1), the hypotheses of Corollary 5.2 as well as under the convergence hypothesis (6.1) on $M_{h} f$.

We recall that, in order to simplify the notation, we have denoted by $(\cdot, \cdot)_{L^{2}}$ the inner product in $\left(L^{2}\left(\Omega_{\varepsilon}\right)\right)^{3}$. We also recall that if $v^{*}=\left(v_{1}^{*}, v_{2}^{*}\right)=M_{h}\left(v_{1}^{*}, v_{2}^{*}, 0\right)$ belongs to $V_{0}$, then the vector $M_{1} v^{*}=\left(v_{1}^{*}, v_{2}^{*}, \frac{x_{3}}{g} v^{*} \cdot \nabla_{h} g\right)$ belongs to $V_{\varepsilon}$.

Before stating the convergence result, we want to obtain a convenient variational equation satisfied by $M_{1}\left(u-v^{0}\right)$, where $u$ and $v^{0}$ are solutions of the equations (1.2) and (6.5). A straightforward computation shows that the solution $v^{0}$ of (6.3) satisfies the following 
equality, for any $v^{*} \in V_{0}$,

$$
\begin{aligned}
& \left(\partial_{t} M_{h} v^{0}, M_{h} v^{*}\right)_{L^{2}}+\frac{1}{2} E\left(M_{1} v^{0}, M_{1} v^{*}\right)+\left(\left(M_{1} v^{0}\right) \cdot \nabla M_{1} v^{0}, M_{1} v^{*}\right)_{L^{2}} \\
= & \left(f_{0}, v^{*}\right)_{L^{2}}+\sum_{i=1}^{2} \int_{\Omega_{\varepsilon}} x_{3}^{2} \partial_{i}\left(\frac{v^{0} \cdot \nabla_{h} g}{g}\right) \partial_{i}\left(\frac{v^{*} \cdot \nabla_{h} g}{g}\right) d x \\
& +\int_{\Omega_{\varepsilon}} x_{3}^{2}\left(\frac{v^{0} \cdot \nabla_{h} g}{g}\right)^{2}\left(\frac{v^{*} \cdot \nabla_{h} g}{g}\right) d x+\sum_{i=1}^{2} \int_{\Omega_{\varepsilon}} x_{3}^{2} v_{i}^{0}\left(\partial_{i}\left(\frac{v^{0} \cdot \nabla_{h} g}{g}\right)\right)\left(\frac{v^{*} \cdot \nabla_{h} g}{g}\right) d x .
\end{aligned}
$$

Taking the inner product in $\left(L^{2}\left(\Omega_{\varepsilon}\right)\right)^{3}$ of the equations (1.2) with $M_{1} v^{*}$, where $v^{*} \in V_{0}$ and applying the Green formula (2.17), one shows, after a short computation, that the solution $u(t)$ of the Navier-Stokes equations (1.2) satisfies the following equality, for any $v^{*} \in V_{0}$,

$$
\begin{aligned}
\left(\partial_{t} M_{h} u, M_{h} v^{*}\right)_{L^{2}} & +\frac{1}{2} E\left(M_{1} u, M_{1} v^{*}\right)+\left(u \cdot \nabla u, M_{1} v^{*}\right)_{L^{2}} \\
= & \left(M_{h} f_{\varepsilon}, v^{*}\right)_{L^{2}}+\int_{\Omega_{\varepsilon}} x_{3} f_{3}\left(\frac{v^{*} \cdot \nabla_{h} g}{g}\right) d x \\
& -\int_{\Omega_{\varepsilon}} x_{3} g^{-1} \partial_{t} u_{3}\left(v^{*} \cdot \nabla_{h} g\right) d x-\frac{1}{2} E\left(\left(I-M_{1}\right) u, M_{1} v^{*}\right) .
\end{aligned}
$$

Subtracting (6.16) from (6.17), we get, for any $v^{*} \in V_{0}$,

$$
\begin{aligned}
& \left(\partial_{t} M_{h}\left(u-v^{0}\right), M_{h} v^{*}\right)_{L^{2}}+\frac{1}{2} E\left(M_{1}\left(u-v^{0}\right), M_{1} v^{*}\right)+\left(u \cdot \nabla u-\left(M_{1} v^{0}\right) \cdot \nabla M_{1} v^{0}, M_{1} v^{*}\right)_{L^{2}} \\
& =\left(M_{h}\left(f_{\varepsilon}-f_{0}\right), v^{*}\right)_{L^{2}}+\int_{\Omega_{\varepsilon}} x_{3} f_{3}\left(\frac{v^{*} \cdot \nabla_{h} g}{g}\right) d x-\int_{\Omega_{\varepsilon}} x_{3} g^{-1} \partial_{t} u_{3}\left(v^{*} \cdot \nabla_{h} g\right) d x \\
& \quad-\frac{1}{2} E\left(\left(I-M_{1}\right) u, M_{1} v^{*}\right)-\sum_{i=1}^{2} \int_{\Omega_{\varepsilon}} x_{3}^{2} \partial_{i}\left(\frac{v^{0} \cdot \nabla_{h} g}{g}\right) \partial_{i}\left(\frac{v^{*} \cdot \nabla_{h} g}{g}\right) d x \\
& \quad-\int_{\Omega_{\varepsilon}} x_{3}^{2}\left(\frac{v^{0} \cdot \nabla_{h} g}{g}\right)^{2}\left(\frac{v^{*} \cdot \nabla_{h} g}{g}\right) d x-\sum_{i=1}^{2} \int_{\Omega_{\varepsilon}} x_{3}^{2} v_{i}^{0}\left(\partial_{i}\left(\frac{v^{0} \cdot \nabla_{h} g}{g}\right)\right)\left(\frac{v^{*} \cdot \nabla_{h} g}{g}\right) d x .
\end{aligned}
$$

We next show that $E\left(\left(I-M_{1}\right) u, M_{1} v^{*}\right)$ is a small term. As in the previous sections, we set $w=\left(I-M_{1}\right) u$ and $v=M_{1} u$. We notice that $\partial_{3} v_{i}^{*}=0$ for $i=1,2$ and that $v_{3}^{*}=\frac{x_{3}}{g} v^{*} \cdot \nabla_{h} g$. Using these properties together with the fact that $w$ and $M_{1} v^{*}$ are divergence-free, we obtain:

$$
\begin{aligned}
E\left(\left(I-M_{1}\right) u, M_{1} v^{*}\right)= & \sum_{i, j=1}^{2} \int_{\Omega_{\varepsilon}}\left(\partial_{j} w_{i}+\partial_{i} w_{j}\right)\left(\partial_{j} v_{i}^{*}+\partial_{i} v_{j}^{*}\right) d x \\
& +4 \int_{\Omega_{\varepsilon}}\left(\partial_{1} w_{1}+\partial_{2} w_{2}\right)\left(\partial_{1} v_{1}^{*}+\partial_{2} v_{2}^{*}\right) d x \\
& +2 \sum_{i=1}^{2} \int_{\Omega_{\varepsilon}}\left(\partial_{3} w_{i}+\partial_{i} w_{3}\right) x_{3} \partial_{i}\left(\frac{1}{g} v^{*} \cdot \nabla_{h} g\right) d x
\end{aligned}
$$


In order to estimate the first two terms in the right hand side of the equality (6.19), we use several times the following equality, valid for any smooth enough functions $h\left(x_{1}, x_{2}, x_{3}\right)$ and $l\left(x_{1}, x_{2}\right)$, with $M h=0$. Replacing $\varphi$ by $h$ in the equality (3.12), we at once obtain for $i=1,2$ (since $M h$ vanishes),

$$
\int_{\Omega_{\varepsilon}} \partial_{i} h\left(x_{1}, x_{2}, x_{3}\right) l\left(x_{1}, x_{2}\right) d x=-\int_{\Omega_{\varepsilon}}\left(\frac{\partial_{i} g}{g}\right) l\left(x_{1}, x_{2}\right) h\left(x_{1}, x_{2}, \varepsilon g\left(x_{1}, x_{2}\right)\right) d x .
$$

In the above equality (6.20), we replace $h$ by $w_{i}$ or $w_{j}$ and $l\left(x_{1}, x_{2}\right)$ by $\left(\partial_{j} v_{i}^{*}+\partial_{i} v_{j}^{*}\right)$ or $\left(\partial_{1} v_{1}^{*}+\partial_{2} v_{2}^{*}\right)$. Thus, applying moreover Lemma 3.3, we deduce from the equality (6.19) that, for any $v^{*} \in V_{0}$,

$$
\left|E\left(\left(I-M_{1}\right) u, M_{1} v^{*}\right)\right| \leq C\left(\varepsilon \sum_{i=1}^{2}\left(\left\|\partial_{3} w_{i}\right\|_{L^{2}}+\left\|\partial_{i} w_{3}\right\|_{L^{2}}\right)\left\|v^{*}\right\|_{H^{1}}\right) \leq C \varepsilon\|\nabla w\|_{L^{2}}\left\|v^{*}\right\|_{H^{1}} .
$$

We are now able to state the following comparison result.

Theorem 6.1. There exists $\varepsilon_{3}>0$ such that, if the initial data $u(0)$ and the forcing term $f$ in (1.2) satisfy the hypotheses of Corollary 5.2 and if moreover $M_{h} f_{\varepsilon}$ converges to a function $f_{0} \in L^{2}\left(\mathbb{T}^{2}\right)$ in the sense of (6.1), then we have the following estimates, for $0<\varepsilon \leq \varepsilon_{3}$,

$$
\left\|S_{0}(t) M_{h} u(0)-M_{h} u(t)\right\|_{L^{2}\left(\mathbb{T}^{2}\right)}^{2} \leq C(1+t)\left(\delta_{1}(\varepsilon)+\delta_{3}(\varepsilon)+\varepsilon^{2}\right) \exp (C(t+1)),
$$

and

$$
\left\|M_{1} S_{0}(t) M_{h} u(0)-u(t)\right\|_{L^{2}}^{2} \leq C \varepsilon(1+t)\left(\delta_{1}(\varepsilon)+\delta_{3}(\varepsilon)+\varepsilon^{2}\right) \exp (C(t+1)) .
$$

Proof. The proof of this theorem consists in two steps.

1) Proof of the inequality (6.22).

Let $v^{0}$ denote the function $S_{0}(t) M_{h} u(0)$. Replacing $v^{*}$ by $M_{h} u-v^{0}$ in (6.18) and taking into account the estimate (6.21) as well as the Korn inequality (2.2), we derive from the inequality (6.18) that, for $t \geq 0$ and $0<\varepsilon \leq \varepsilon_{2}$, where $\varepsilon_{2}$ is small enough,

$$
\begin{aligned}
\frac{\mathrm{d}}{\mathrm{d} t}\left\|M_{h}\left(u-v^{0}\right)(t)\right\|_{L^{2}}^{2}+\frac{c_{0}}{2}\left\|M_{1}\left(u-v^{0}\right)\right\|_{H^{1}}^{2} & \leq C\left[\left\|M_{h}\left(f_{\varepsilon}-f_{0}\right)\right\|_{L^{\infty}\left(L^{2}\right)}^{2}+\varepsilon^{2}\left\|f_{3}\right\|_{L^{\infty}\left(L^{2}\right)}^{2}+N_{1}\right. \\
& +N_{2}+\varepsilon^{2}\left\|\frac{\partial u_{3}}{\partial t}\right\|_{L^{2}}^{2}+\varepsilon^{2}\|\nabla w\|_{L^{2}}^{2}+\varepsilon^{4}\left\|v^{0}\right\|_{H^{1}}^{2},
\end{aligned}
$$

where

$$
\begin{aligned}
& N_{1}=\mid\left(u \cdot \nabla u-\left(M_{1} v^{0}\right)\right.\left.\cdot \nabla M_{1} v^{0}, M_{1}\left(u-v^{0}\right)\right)_{L^{2}} \mid \\
& N_{2}=\left|\int_{\Omega_{\varepsilon}} x_{3}^{2}\left(\frac{v^{0} \cdot \nabla_{h} g}{g}\right)^{2}\left(\frac{\left(M_{h} u-v^{0}\right) \cdot \nabla_{h} g}{g}\right) d x\right| \\
& \quad+\left|\sum_{i=1}^{2} \int_{\Omega_{\varepsilon}} x_{3}^{2} v_{i}^{0}\left(\partial_{i}\left(\frac{v^{0} \cdot \nabla_{h} g}{g}\right)\right)\left(\frac{\left(M_{h} u-v^{0}\right) \cdot \nabla_{h} g}{g}\right) d x\right| .
\end{aligned}
$$


It remains to consider the terms $N_{1}$ and $N_{2}$. We begin by estimating the term $N_{2}$. Applying the Hölder inequality together with a two-dimensional Gagliardo-Nirenberg inequality, we get,

$$
\begin{gathered}
N_{2} \leq C \varepsilon^{2}\left(\left\|v^{0}\right\|_{L^{4}}^{2}\left\|M_{1}\left(u-v^{0}\right)\right\|_{L^{2}}+\left\|v^{0}\right\|_{L^{4}}\left\|v^{0}\right\|_{H^{1}}\left\|M_{1}\left(u-v^{0}\right)\right\|_{L^{4}}\right) \\
\leq C \varepsilon^{3 / 2}\left(\left\|v^{0}\right\|_{L^{2}}\left\|v^{0}\right\|_{H^{1}}\left\|M_{1}\left(u-v^{0}\right)\right\|_{L^{2}}\right. \\
\left.\quad+\left\|v^{0}\right\|_{L^{2}}^{1 / 2}\left\|v^{0}\right\|_{H^{1}}^{3 / 2}\left\|M_{1}\left(u-v^{0}\right)\right\|_{L^{2}}^{1 / 2}\left\|M_{1}\left(u-v^{0}\right)\right\|_{H^{1}}^{1 / 2}\right) \\
\leq C\left(\varepsilon^{3 / 2}\left\|v^{0}\right\|_{L^{2}}\left\|v^{0}\right\|_{H^{1}}\left\|M_{1}\left(u-v^{0}\right)\right\|_{L^{2}}+\varepsilon^{2}\left\|v^{0}\right\|_{L^{2}}\left\|v^{0}\right\|_{H^{1}}^{2}\right. \\
\left.+\varepsilon\left\|v^{0}\right\|_{H^{1}}\left\|M_{1}\left(u-v^{0}\right)\right\|_{L^{2}}\left\|M_{1}\left(u-v^{0}\right)\right\|_{H^{1}}\right) .
\end{gathered}
$$

We next estimate the term $N_{1}$. We notice that, due to the orthogonality property between $(I-M) u$ and any function independent of $x_{3}$, the term $\left(w \cdot \nabla v, M_{1}\left(u-v^{0}\right)\right)_{L^{2}}$ reduces to the term

$$
\sum_{i=1}^{3} \int_{\Omega_{\varepsilon}} g^{-1} x_{3} w_{i} \partial_{i} v_{3}\left(\left(v_{h}-v^{0}\right) \cdot \nabla_{h} g\right) d x
$$

and thus we obtain the following equality:

$$
\begin{aligned}
N_{1}= & \left|\left(w \cdot \nabla w+v \cdot \nabla w, M_{1}\left(u-v^{0}\right)\right)_{L^{2}}\right|+\left|\left(M_{1}\left(u-v^{0}\right) \cdot \nabla M_{1} v^{0}, M_{1}\left(u-v^{0}\right)\right)_{L^{2}}\right| \\
& +\sum_{i=1}^{3} \int_{\Omega_{\varepsilon}} g^{-1} x_{3} w_{i} \partial_{i} v_{3}\left(\left(v_{h}-v^{0}\right) \cdot \nabla_{h} g\right) d x .
\end{aligned}
$$

Applying the Gagliardo-Nirenberg inequalities (3.27) and (3.28), we estimate the first term of the right-hand side of (6.26) as follows,

$$
\begin{aligned}
\left|\left(w \cdot \nabla w, M_{1}\left(u-v^{0}\right)\right)_{L^{2}}\right| & \leq\|w\|_{L^{4}}\|\nabla w\|_{L^{4}}\left\|M_{1}\left(u-v^{0}\right)\right\|_{L^{2}} \\
& \leq C \varepsilon^{1 / 2}\|w\|_{H^{1}}\|w\|_{H^{2}}\left\|M_{1}\left(u-v^{0}\right)\right\|_{L^{2}} \\
& \leq C \varepsilon^{2}\|w\|_{H^{2}}^{2}+C \varepsilon^{-1}\|w\|_{H^{1}}^{2}\left\|M_{1}\left(u-v^{0}\right)\right\|_{L^{2}}^{2} .
\end{aligned}
$$

We next estimate the term $\left|\left(v \cdot \nabla w, M_{1}\left(u-v^{0}\right)\right)_{L^{2}}\right|$. By the identity (6.20), we obtain

$$
\sum_{i, j=1}^{2} \int_{\Omega_{\varepsilon}} v_{i} \partial_{i} w_{j}\left(M_{1}\left(u-v^{0}\right)\right)_{j} d x=-\sum_{i, j=1}^{2} \int_{\Omega_{\varepsilon}}\left(\frac{\partial_{i} g}{g}\right)\left(v_{i}\left(M_{1}\left(u-v^{0}\right)\right)_{j}\right)\left(x_{h}\right) w_{j}\left(x_{h}, \varepsilon g\left(x_{h}\right)\right) d x,
$$

Thus we can write

$$
\begin{aligned}
\left(v \cdot \nabla w, M_{1}\left(u-v^{0}\right)\right)_{L^{2}} & =-\sum_{i, j=1}^{2} \int_{\Omega_{\varepsilon}}\left(\frac{\partial_{i} g}{g}\right)\left(v_{i}\left(M_{1}\left(u-v^{0}\right)\right)_{j}\right)\left(x_{h}\right) w_{j}\left(x_{h}, \varepsilon g\left(x_{h}\right)\right) d x \\
& +\sum_{i=1}^{2} \int_{\Omega_{\varepsilon}} v_{i} \partial_{i} w_{3} x_{3} g^{-1}\left(\left(v_{h}-v^{0}\right) \cdot \nabla_{h} g\right) d x \\
& +\sum_{j=1}^{3} \int_{\Omega_{\varepsilon}} x_{3} g^{-1}\left(v_{h} \cdot \nabla_{h} g\right) \partial_{3} w_{j}\left(M_{1}\left(u-v^{0}\right)\right)_{j} d x
\end{aligned}
$$

In order to estimate the first term in the right hand side of the above equality, we apply Lemma 3.3 and the two-dimensional Gagliardo-Nirenberg inequality. To bound the two 
other terms in the right hand side of the above equality, we simply use the Hölder inequality and again the two-dimensional Gagliardo-Nirenberg inequality. We thus get

$$
\begin{aligned}
\left|\left(v \cdot \nabla w, M_{1}\left(u-v^{0}\right)\right)_{L^{2}}\right| & \leq C \varepsilon\left\|v_{h}\right\|_{L^{4}}\left\|M_{1}\left(u-v^{0}\right)\right\|_{L^{4}}\|w\|_{H^{1}} \\
& \leq C \varepsilon^{1 / 2}\left\|v_{h}\right\|_{L^{2}}^{1 / 2}\left\|v_{h}\right\|_{H^{1}}^{1 / 2}\left\|M_{1}\left(u-v^{0}\right)\right\|_{L^{2}}^{1 / 2}\left\|M_{1}\left(u-v^{0}\right)\right\|_{H^{1}}^{1 / 2}\|w\|_{H^{1}}
\end{aligned}
$$

Likewise, using again the two-dimensional Gagliardo-Nirenberg inequality and applying Lemma 3.10, we obtain

$$
\begin{aligned}
&\left|\sum_{i=1}^{3} \int_{\Omega_{\varepsilon}} g^{-1} x_{3} w_{i} \partial_{i} v_{3}\left(\left(v_{h}-v^{0}\right) \cdot \nabla_{h} g\right) d x\right| \leq C\left(\varepsilon\left\|v_{h}\right\|_{L^{4}}\|w\|_{L^{2}}+\varepsilon^{2}\left\|v_{h}\right\|_{H^{1}}\|w\|_{L^{4}}\right) \\
& \times\left\|M_{1}\left(u-v^{0}\right)\right\|_{L^{4}} \\
& \leq C\left(\varepsilon^{1 / 2}\left\|v_{h}\right\|_{L^{2}}^{\frac{1}{2}}\left\|v_{h}\right\|_{H^{1}}^{\frac{1}{2}}\|w\|_{L^{2}}+\varepsilon^{2}\left\|v_{h}\right\|_{H^{1}}\|w\|_{H^{1}}\right) \\
& \times\left\|M_{1}\left(u-v^{0}\right)\right\|_{L^{2}}^{1 / 2}\left\|M_{1}\left(u-v^{0}\right)\right\|_{H^{1}}^{1 / 2}
\end{aligned}
$$

Finally, we estimate the term $\left|\left(M_{1}\left(u-v^{0}\right) \cdot \nabla M_{1} v^{0}, M_{1}\left(u-v^{0}\right)\right)_{L^{2}}\right|$. As above, we use the Hölder inequality and a two-dimensional Gagliardo-Nirenberg inequality to get

$$
\begin{aligned}
\left|\left(M_{1}\left(u-v^{0}\right) \cdot \nabla M_{1} v^{0}, M_{1}\left(u-v^{0}\right)\right)_{L^{2}}\right| & \leq C \varepsilon^{1 / 2}\left\|v^{0}\right\|_{H^{1}\left(\mathbb{T}^{2}\right)}\left\|M_{1}\left(u-v^{0}\right)\right\|_{L^{4}}^{2} \\
& \leq C\left\|v^{0}\right\|_{H^{1}\left(\mathbb{T}^{2}\right)}\left\|M_{1}\left(u-v^{0}\right)\right\|_{L^{2}}\left\|M_{1}\left(u-v^{0}\right)\right\|_{H^{1}} .
\end{aligned}
$$

We thus have estimated all the terms contained in $N_{1}$ and $N_{2}$. Using several times the Young inequality, we deduce from the estimates (6.24), (6.25), and the inequalities (6.26) to (6.27) that, for $t \geq 0$,

$$
\begin{aligned}
\frac{\mathrm{d}}{\mathrm{d} t}\left\|M_{h}\left(u-v^{0}\right)(t)\right\|_{L^{2}}^{2}+\frac{c_{0}}{4}\left\|M_{1}\left(u-v^{0}\right)\right\|_{H^{1}}^{2} \leq & C\left[\left\|M_{h}\left(f_{\varepsilon}-f_{0}\right)\right\|_{L^{\infty}\left(L^{2}\right)}^{2}+\varepsilon^{2}\left\|f_{3}\right\|_{L^{\infty}\left(L^{2}\right)}^{2}\right. \\
& +\varepsilon^{3}\left\|v^{0}\right\|_{L^{2}}^{2}\left\|v^{0}\right\|_{H^{1}}^{2}+\varepsilon^{2}\left\|v^{0}\right\|_{L^{2}}\left\|v^{0}\right\|_{H^{1}}^{2} \\
& +\varepsilon^{4}\left\|v^{0}\right\|_{H^{1}}^{2}+\varepsilon^{2}\|u\|_{H^{2}}^{2}+\varepsilon^{4}\left\|v_{h}\right\|_{H^{1}}^{2}\|w\|_{H^{1}}^{2} \\
& +\varepsilon^{2}\left\|\frac{\partial u_{3}}{\partial t}\right\|_{L^{2}}^{2}+\varepsilon\left\|v_{h}\right\|_{L^{2}}\left\|v_{h}\right\|_{H^{1}}\|w\|_{H^{1}}^{2} \\
& +\varepsilon^{-1}\|w\|_{H^{1}}^{2}\left\|M_{1}\left(u-v^{0}\right)\right\|_{L^{2}}^{2} \\
& \left.+\left\|v^{0}\right\|_{H^{1}\left(\mathbb{T}^{2}\right)}^{2}\left\|M_{1}\left(u-v^{0}\right)\right\|_{L^{2}}^{2}\right] .
\end{aligned}
$$

Integrating the inequality (6.28) from 0 to $t$ and taking into account the estimates given in Theorem 5.1 and in Corollary 5.2, we obtain, for $0<\varepsilon \leq \varepsilon_{3}$ and $t \geq 0$, where $\varepsilon_{3}$ is chosen 
small enough,

$$
\begin{aligned}
\left\|M_{1}\left(u-v^{0}\right)(t)\right\|_{L^{2}}^{2}+\frac{c_{0}}{4} \int_{0}^{t}\left\|M_{1}\left(u-v^{0}\right)(s)\right\|_{H^{1}}^{2} d s & \leq C\left[\varepsilon(1+t)\left(\delta_{1}(\varepsilon)+\delta_{3}(\varepsilon)+\varepsilon^{2}\right)\right. \\
& +\int_{0}^{t} \varepsilon^{-1}\|u(s)\|_{H^{1}}^{2}\left\|M_{1}\left(u-v^{0}\right)(s)\right\|_{L^{2}}^{2} d s \\
& \left.+\int_{0}^{t}\left\|v^{0}(s)\right\|_{H^{1}\left(\mathbb{T}^{2}\right)}^{2}\left\|M_{1}\left(u-v^{0}\right)(s)\right\|_{L^{2}}^{2} d s\right] .
\end{aligned}
$$

Finally, applying the Gronwall inequality, we deduce from the inequality (6.29), from Theorem 5.1 and the estimate (6.11) that, for $0<\varepsilon \leq \varepsilon_{3}$ and $t \geq 0$,

$$
\left\|\left(M_{h} u-v^{0}\right)(t)\right\|_{L^{2}\left(\mathbb{T}^{2}\right)}^{2} \leq C(1+t)\left(\delta_{1}(\varepsilon)+\delta_{3}(\varepsilon)+\varepsilon^{2}\right) \exp (C(t+1)),
$$

which implies the inequality (6.22).

2) Proof of the inequality (6.23). We remark that

$$
M_{1} S_{0}(t) M_{h} u(0)-u(t)=M_{1}\left(S_{0}(t) M_{h} u(0)-M_{h} u(t)\right)+\left(I-M_{1}\right) u(t) .
$$

According to Proposition 3.6 and to Theorem 5.1, the vector $w(t)=\left(I-M_{1}\right) u(t)$ satisfies the following inequality

$$
\|w(t)\|_{L^{2}}^{2} \leq C \varepsilon\left(\delta_{1}(\varepsilon)+\varepsilon^{2}\right)
$$

The estimate (6.23) is then a direct consequence of (6.22), (6.30), and (6.31).

We conclude this section by remarking that Theorem 1.2 follows directly from Theorem 6.1 together with the Lipschitz dependency (6.8) of the solutions of the equations (6.5).

\section{LOCAL ATTRACTOR}

Like in [33], [34] and [3], we are now going to introduce a (local) attractor and show that this (local) attractor is actually the compact global attractor of all weak Leray-Hopf solutions. Without loss of generality, we suppose that the viscosity $\nu$ is equal to 1 . To simplify the statements, we assume in this section that the forcing term $f \equiv f_{\varepsilon}$ does not depend on the time variable and satisfies the conditions (5.1). We also suppose that $0<\varepsilon \leq \varepsilon_{2}$ and that the constants given in (5.1) satisfy the condition (5.18) as well as the following additional property

$$
\frac{4 c_{1}}{c_{0}}\left(K_{0}^{2}+K_{1}^{2}\right) \leq \kappa_{0}^{2}
$$

where the constant $c_{1}$ is given in (5.3). According to Theorem 1.1, for any $u_{0}$ satisfying the conditions (5.1) and for $0<\varepsilon \leq \varepsilon_{2}$, there exists a unique global strong solution $u(t) \equiv S_{\varepsilon}(t) u_{0} \in C^{0}\left([0,+\infty), V_{\varepsilon}\right)$ of the Navier-Stokes equations (1.2). Actually, as $f_{\varepsilon}$ is independent of the time variable $t$, the solution $u$ is also a classical solution of the Navier-Stokes equations (1.2) and belongs to the space $C^{0}\left([0,+\infty), V_{\varepsilon}\right) \cap C^{1}\left((0,+\infty), H_{\varepsilon}\right) \cap$ $C^{0}\left((0,+\infty), D\left(A_{\varepsilon}\right)\right)$. 
We next define the sets

$$
\begin{aligned}
\mathcal{B}_{0, \varepsilon} & =\left\{u_{0} \in V_{\varepsilon} \mid\left\|u_{0}\right\|_{H^{1}} \leq \kappa_{1} \varepsilon^{-1 / 2},\left\|M_{h} u_{0}\right\|_{L^{2}} \leq \kappa_{0}\right\}, \\
\mathcal{B}_{\varepsilon} & =\bigcup_{t \geq 0} S_{\varepsilon}(t) \mathcal{B}_{0, \varepsilon}
\end{aligned}
$$

Due to Theorem 1.1, the set $\mathcal{B}_{\varepsilon}$ is bounded in $V_{\varepsilon}$ and the nonlinear semiflow $S_{\varepsilon}(t)$ is welldefined on $\mathcal{B}_{\varepsilon}$. Moreover, $\mathcal{B}_{\varepsilon}$ is positively invariant under $S_{\varepsilon}(t)$, for $t \geq 0$. As in the case of the Navier-Stokes equations with classical boundary conditions, for any $u_{0} \in \mathcal{B}_{\varepsilon}, S_{\varepsilon}(t) u_{0}$ belongs to $C^{0}\left((0,+\infty), D\left(A_{\varepsilon}\right)\right)$. Since $D\left(A_{\varepsilon}\right)$ is compactly embedded in $V_{\varepsilon}$, for $t>0$, the map $S_{\varepsilon}(t)$ is compact from $V_{\varepsilon}$ into itself, for $t>0$. Thus, the $\omega$-limit set of $\mathcal{B}_{\varepsilon}$,

$$
\mathcal{A}_{\varepsilon}=\bigcap_{\tau \geq 0} \widehat{\bigcup}_{t \geq \tau} S_{\varepsilon}(t) \mathcal{B}_{\varepsilon}{ }^{V_{\varepsilon}}
$$

is a well-defined, nonempty, compact set and attracts $\mathcal{B}_{\varepsilon}$ (see [10], Chapter 2). The set $\mathcal{A}_{\varepsilon}$ is the compact global attractor of the restriction of $S_{\varepsilon}(t)$ to $\mathcal{B}_{\varepsilon}$.

Furthermore, by the properties (5.19) and (5.23), the attractor satisfies the following inclusion

$$
\mathcal{A}_{\varepsilon} \subset B_{H_{\varepsilon}}(0, C \mathcal{R}) \cap B_{V_{\varepsilon}}\left(0, C_{3}\left(\|f\|_{L^{\infty}\left(L^{2}\right)}^{2}+\varepsilon^{-1} \mathcal{R}^{4}\right)^{1 / 2}\right),
$$

where $\mathcal{R}^{2}=\left\|M_{h} f\right\|_{L^{2}}^{2}+\varepsilon^{2}\left\|\left(I-M_{h}\right) f\right\|_{L^{2}}^{2}$.

We next show, like in [33], [34] and [3], that $\mathcal{A}_{\varepsilon}$ is the global attractor of the weak Leray-Hopf solutions of (1.2). We begin by recalling the definition of these weak solutions.

We denote by $C_{w}^{0}\left([0, T] ; H_{\varepsilon}\right)$ the subspace of $L^{\infty}\left((0, T) ; H_{\varepsilon}\right)$ consisting of all functions which are weakly continuous, that is, for each $h \in H_{\varepsilon}$, the mapping $t \rightarrow(u(t), h)$ is continuous.

A weak Leray-Hopf solution $u(t)$ on the time interval $[0, T]$ is a function $u($.$) in the$ space $L_{l o c}^{2}\left((0, T) ; V_{\varepsilon}\right) \cap L^{\infty}\left((0, T) ; H_{\varepsilon}\right) \cap C_{w}^{0}\left([0, T] ; H_{\varepsilon}\right)$, with $\partial_{t} u \in L_{l o c}^{1}\left((0, T) ; V_{\varepsilon}^{\prime}\right)$, such that $u(0)=u_{0}$ holds in the weak sense, the equation

$$
\left(u(t)-u\left(t_{0}\right), u^{*}\right)_{L^{2}}+\frac{1}{2} \int_{t_{0}}^{t} E\left(u, u^{*}\right) d s+\int_{t_{0}}^{t}\left(\sum_{j=1}^{3} u_{j} \partial_{j} u, u^{*}\right)_{L^{2}} d s=\int_{t_{0}}^{t}\left(f_{\varepsilon}, u^{*}\right)_{L^{2}} d s,
$$

is satisfied, for all $t \geq t_{0} \geq 0$ and $u^{*} \in V_{\varepsilon}$, and the energy inequality

$$
\frac{1}{2}\|u(t)\|_{L^{2}}^{2}+\frac{1}{2} \int_{t_{0}}^{t} E(u(s), u(s)) d s \leq \frac{1}{2}\|u(0)\|_{L^{2}}^{2}+\int_{t_{0}}^{t}\left(f_{\varepsilon}, u(s)\right)_{L^{2}} d s
$$

holds for almost all $t$ and almost all $t_{0}$ with $0<t_{0}<t$ and also for $t_{0}=0$ (see for example [21], [42], [6] or also [39]).

Since $E(\cdot, \cdot)$ is a positive bilinear coercive form (see Section 2 for further details), by using a Galerkin method, we can argue, exactly as in [21], [42] or [6], to show that, for any $u_{0} \in H_{\varepsilon}$, the Navier-Stokes equations (1.2) admit a weak Leray-Hopf global solution $u(t)$ in $[0,+\infty)$. We also notice that $u(t) \in V_{\varepsilon}$ for $t \in \mathcal{F}_{u}^{T}$, where $\mathcal{F}_{u}^{T} \subset[0, T]$ is a measurable set of full measure. 
In their paper of 1987, Foiaş and Temam $([8])$ introduced the set $J_{\varepsilon}$ of all weak LerayHopf solutions existing in $(-\infty,+\infty)$ and bounded in $L^{\infty}\left((-\infty,+\infty) ; H_{\varepsilon}\right.$. This set is not empty since it contains $\mathcal{A}_{\varepsilon}$. Foias and Temam ([8]) also showed that this set $J_{\varepsilon}$ is compact in $H_{\varepsilon}^{\text {weak }}$ and that, for any weak Leray-Hopf solution $u(t)$ in $(0,+\infty), u(t) \rightarrow J_{\varepsilon}$ in $H_{\varepsilon}^{\text {weak }}$ as $t \rightarrow+\infty$. In [38], Sell proved that this set $J_{\varepsilon}$ is an attractor. We next show that $J_{\varepsilon}=\mathcal{A}_{\varepsilon}$, for $0<\varepsilon \leq \varepsilon_{3}$, where $\varepsilon_{3}>0$ is small enough. We use the same arguments as in [42, Theorem 3.12, Chap.3], [33], [34] and [3].

Theorem 7.1. Assume that the conditions (7.1) and (5.1) hold, and that $f_{\varepsilon} \in L^{2}\left(\Omega_{\varepsilon}\right)^{3}$ satisfies (5.1). Then, there exist a positive number $\varepsilon_{3} \leq \varepsilon_{2}$ and, for any $r>0$, for any $0<\varepsilon \leq \varepsilon_{3}$, a time $T(\varepsilon, r) \geq 0$ such that, for any weak Leray-Hopf solution u(t) of (1.2), with $\|u(0) \mid\|_{L^{2}} \leq r$, there is a positive time $t_{1}, 0<t_{1} \leq T(\varepsilon, r)$ so that $u(t) \in \mathcal{B}_{\varepsilon}$ for $t \geq t_{1}$. In particular, $u(t)$ is a classical solution of (1.2), for $t \geq t_{1}$ and $J_{\varepsilon}=\mathcal{A}_{\varepsilon}$.

Proof. Let $u(t)$ be weak Leray-Hopf solution of the equations (1.2). Arguing as in Section 3 (see (5.3)), we deduce from the energy inequality (7.3) and from the Korn inequality (2.2), that, for $t \geq 0$, for $0 \leq \varepsilon \leq \varepsilon_{2}$,

$$
\frac{1}{t} \int_{0}^{t}\|u(s)\|_{H^{1}}^{2} d s \leq \frac{2}{t c_{0}}\|u(0)\|_{L^{2}}^{2}+\frac{2 c_{1}}{c_{0}}\left(\left\|M_{h} f_{\varepsilon}\right\|_{L^{2}}^{2}+\varepsilon^{2}\left\|\left(I-M_{h}\right) f_{\varepsilon}\right\|_{L^{2}}^{2}\right),
$$

and therefore we have,

$$
\frac{1}{t} \int_{0}^{t}\|u(s)\|_{H^{1}}^{2} d s \leq \frac{3 c_{1}}{c_{0}}\left(K_{0}^{2}+\varepsilon K_{1}^{2}\right), \quad \text { for all } t \geq T(\varepsilon, r),
$$

where

$$
T(\varepsilon, r)=\frac{2 r^{2}}{c_{1}\left(K_{0}^{2}+\varepsilon^{2} K_{1}^{2}\right)} .
$$

The estimate (7.4) and the property (7.1) imply that,

$$
\frac{1}{t} \int_{0}^{t}\|u(s)\|_{H^{1}}^{2} d s \leq \frac{3 c_{1}}{c_{0}}\left(K_{0}^{2}+\varepsilon K_{1}^{2}\right)<\kappa_{0}^{2}, \quad \text { for all } t \geq T(\varepsilon, r),
$$

We can choose $\varepsilon_{3}>0$, with $\varepsilon_{3} \leq \varepsilon_{2}$ such that $\frac{3 c_{1}}{c_{0}}\left(K_{0}^{2}+\varepsilon_{3} K_{1}^{2}\right)<\kappa_{1}^{2} \varepsilon_{3}^{-1}$. Thus, we have, for $0<\varepsilon \leq \varepsilon_{3}$,

$$
\frac{1}{t} \int_{0}^{t}\|u(s)\|_{H^{1}}^{2} d s<\min \left(\kappa_{1}^{2} \varepsilon^{-1}, \kappa_{0}^{2}\right), \quad \text { for all } t \geq T(\varepsilon, r) .
$$

Therefore, there exists a subset $\mathcal{F}_{0} \subset[0, T(\varepsilon, r)]$ of positive measure such that

$$
\|u(t)\|_{H^{1}}^{2} \leq \min \left(\kappa_{1}^{2} \varepsilon^{-1}, \kappa_{0}^{2}\right) .
$$

Since $\mathcal{F}_{u}^{T(\varepsilon, r)}$ is a set of full measure in $[0, T(\varepsilon, r)]$, it follows that $\mathcal{F}_{u}^{T(\varepsilon, r)} \cap \mathcal{F}_{0} \neq \emptyset$. Let $t_{1}$ be a point in this intersection. We deduce now from Theorem 1.1 and from the uniqueness of classical solutions in the class of strong solutions that $u(t)$ is a classical solution of (1.2) for $t \geq t_{1}$ and that $u(t)$ belongs to $\mathcal{B}_{\varepsilon}$, for $t \geq t_{1}$. It also at once follows that $J_{\varepsilon}=\mathcal{A}_{\varepsilon}$.

We remark that, since $\mathcal{A}_{\varepsilon}$ is the global attractor for all weak solutions, it is also the global attractor for all the globally defined strong solutions, including those that satisfy the conditions (1.5). Moreover, $\mathcal{A}_{\varepsilon}$ is a local attractor in $V_{\varepsilon}$. Indeed, by using the compactness 
of $\mathcal{A}_{\varepsilon}$ and the Lipschitz dependence of the solutions with respect to the initial data, one classically shows that there exists a neighbourhood $N_{\varepsilon}$ of $\mathcal{A}_{\varepsilon}$ in $V_{\varepsilon}$, so that, for all initial data in this neighbourhood $N_{\varepsilon}$, the strong solutions exist on $(0,+\infty)$. Then the proof of Theorem 7.1 implies that actually these strong solutions are attracted by $\mathcal{A}_{\varepsilon}$. This implies that $\mathcal{A}_{\varepsilon}$ attracts the neighbourhood $N_{\varepsilon}$ and thus $\mathcal{A}_{\varepsilon}$ is a local attractor in $V_{\varepsilon}$.

It remains to compare the attractor $\mathcal{A}_{\varepsilon}$ with the global attractor $\mathcal{A}_{0}$, when $\varepsilon>0$ is small enough and when $f_{\varepsilon}$ converges to $f_{0}$ in some sense.

Theorem 7.2. Assume that the forcing term $f_{\varepsilon}$ satisfies the hypotheses of Corollary 5.2 and that $M_{h} f_{\varepsilon}$ converges to a limit function $f_{0} \in L^{2}\left(\mathbb{T}^{2}\right)^{2}$ in the sense of $(6.1)$, then the sets $M_{h} \mathcal{A}_{\varepsilon}$ are upper-semicontinuous with respect to $\varepsilon$ in the $L^{2}\left(\mathbb{T}^{2}\right)^{2}$-topology and the attractors $\mathcal{A}_{\varepsilon}$ are also upper-semicontinuous with respect to $\varepsilon$ in the $L^{2}\left(\Omega_{\varepsilon}\right)^{3}$-topology. More precisely, for any $\eta>0$, there exists $\varepsilon_{4}>0$ such that, for $0<\varepsilon \leq \varepsilon_{4}$,

$$
M_{h} \mathcal{A}_{\varepsilon} \subset N_{L^{2}\left(\mathbb{T}^{2}\right)^{2}}\left(\mathcal{A}_{0}, \eta\right), \quad \mathcal{A}_{\varepsilon} \subset N_{L^{2}\left(\Omega_{\varepsilon}\right)^{3}}\left(M_{1} \mathcal{A}_{0}, \varepsilon^{1 / 2} \eta\right),
$$

where $N_{X}(B, r)$ denotes the $r$-neighbourhood of $B$ in the space $X$.

Proof. We first remark that, since $f_{\varepsilon}$ satisfies the hypotheses of Corollary 5.2, we deduce from the property (7.2) that

$$
\mathcal{A}_{\varepsilon} \subset B_{L^{2}\left(\Omega_{\varepsilon}\right)^{3}}\left(0, \bar{K} \varepsilon^{1 / 2}\right) \cap B_{H^{1}\left(\Omega_{\varepsilon}\right)^{3}}\left(0, \bar{K} \varepsilon^{-1 / 2} \delta_{1}(\varepsilon)^{1 / 2}\right), \quad M_{h} \mathcal{A}_{\varepsilon} \subset B_{L^{2}\left(\mathbb{T}^{2}\right)^{2}}(0, \bar{K}),
$$

where $\bar{K}$ is a constant independent of $\varepsilon$.

Since $\mathcal{A}_{0}$ attracts the ball $B_{L^{2}\left(\mathbb{T}^{2}\right)^{2}}(0, \bar{K})$ in $L^{2}\left(\mathbb{T}^{2}\right)^{2}$, for any $\eta>0$, there exists a time $\tau_{0}=\tau_{0}(\eta)$ such that, for $t \geq \tau_{0}$,

$$
S_{0}(t) M_{h} \mathcal{A}_{\varepsilon} \subset N_{L^{2}\left(\mathbb{T}^{2}\right)^{2}}\left(\mathcal{A}_{0}, \frac{\eta}{2}\right) .
$$

We next deduce from the first estimate in Theorem 6.1 that, there exists $\varepsilon_{4}>0$ such that, for $0<\varepsilon \leq \varepsilon_{4}$, for all $u(0) \in \mathcal{A}_{\varepsilon}$,

$$
\left\|S_{0}\left(\tau_{0}\right) M_{h} u(0)-M_{h} u\left(\tau_{0}\right)\right\|_{L^{2}\left(\mathbb{T}^{2}\right)}^{2} \leq \frac{\eta}{2} .
$$

We at once deduce from the properties (7.5) and (7.6) that $M_{h} S_{\varepsilon}\left(\tau_{0}\right) \mathcal{A}_{\varepsilon} \subset N_{L^{2}\left(\mathbb{T}^{2}\right)^{2}}\left(\mathcal{A}_{0}, \eta\right)$. Since $\mathcal{A}_{\varepsilon}$ is invariant by the action of $S_{\varepsilon}\left(\tau_{0}\right)$, it implies that $M_{h} \mathcal{A}_{\varepsilon} \subset N_{L^{2}\left(\mathbb{T}^{2}\right)^{2}}\left(\mathcal{A}_{0}, \eta\right)$. The second assertion of the theorem is proved in the same way and is left to the reader. 


\section{REFERENCES}

[1] J. D. Avrin, Large-eigenvalue global existence and regularity results for the Navier-Stokes equation, J. Differential Equations 127 (1996), 365-390.

[2] V. Busuioc and T.S. Ratiu, The second grade fluid and averaged Euler equations with Navier-slip boundary conditions, Nonlinearity 16 (2003), 1119-1149.

[3] I. Chueshov, G. Raugel and A. Rekalo, Interface boundary value problem for the Navier-Stokes equations in thin two-layer domains, Journal Diff. Equations 208 (2005).

[4] I. Ciuperca, private communication (letter), (1994).

[5] Th. Clopeau, A. Mikelić and R. Robert. On the vanishing viscosity limit for the 2D incompressible Navier-Stokes equations with the friction type boundary conditions. Nonlinearity 11 (1998), no. 6, 1625-1636.

[6] P. Constantin and C. Foiaş, Navier-Stokes equations, University of Chicago Press, Chicago, 1988.

[7] C. Foiaş and G. Prodi, Sur le compartement global des solutions non stationnaires des équations de Navier-Stokes en dimension 2, Rend Sem Mat Univ Padova 39 (1967), 1-34.

[8] C. Foias, R. Temam, The connection between the Navier-Stokes equations, dynamical systems, and turbulence theory, Directions in Partial Differential Equations, Academic Press, New York (1987), 55-73.

[9] H. Fujita and T. Kato, On the Navier-Stokes initial value problem. I, Arch. Rational Mech. Anal. 16 (1964), 269-315.

[10] J. K. Hale, Asymptotic behavior of dissipative systems, Mathematical Surveys and Monographs 25, American Mathematical Society, Providence, RI (1988)

[11] J. K. Hale and G. Raugel, Partial differential equations on thin domains, Differential equations and mathematical physics (Birmingham, AL, 1990), Academic Press, Boston, MA, 1992, pp. 63-97.

[12] _ A damped hyperbolic equation on thin domains, Trans. Amer. Math. Soc. 329 (1992), $185-219$.

[13] _ Reaction-diffusion equation on thin domains, J. Math. Pures Appl. 71 (1992), 33-95.

[14] D. Iftimie, The 3D Navier-Stokes equations seen as a perturbation of the 2D Navier-Stokes equations, Bull. Soc. Math. France 127 (1999), 473-517.

[15] D. Iftimie and G. Raugel, Some results on the Navier-Stokes equations in thin 3D domains, J. Differential Equations 169 (2001), 281-331.

[16] W. Jäger and A. Mikelić. Couette flows over a rough boundary and drag reduction. Comm. Math. Phys. 232 (2003), no. 3, 429-455.

[17] W. Jäger and A. Mikelić. On the roughness-induced effective boundary conditions for an incompressible viscous flow. J. Differential Equations 170 (2001), no. 1, 96-122.

[18] J. Leray, Sur le mouvement d'un liquide visqueux emplissant l'espace, Acta Math. 63 (1934), $193-248$.

[19] C. D. Levermore, M. Oliver and E. Titi Global well-posedness for models of shallow water in a basin with a varying bottom, Indiana Univ. Math. J. 45 (1996), 479-510.

[20] J.-L. Lions, Problèmes aux limites dans les équations aux dérivées partielles, Les Presses de l'Uniersité de Montréal (1965), Montréal.

[21] _ Q Quelques méthodes de résolution des problèmes aux limites non linéaires, Gauthier Villars, Paris, 1969.

[22] J.-L. Lions, R. Temam and S. Wang, Mathematical theory for the coupled atmosphere-ocean models (CAO III), J. Math. Pures Appl., 74, (1995), 105-163.

[23] M. Lopes Filho, H. Nussenzveig Lopes and G. Planas. On the inviscid limit for 2D incompressible flow with Navier friction condition, SIAM J. Math. Analysis, 36, (2005), 1130-1141.

[24] I. Moise, R. Temam, and M. Ziane, Asymptotic analysis of the Navier-Stokes equations in thin domains, Topol. Methods Nonlinear Anal. 10 (1997), 249-282.

[25] S. Montgomery-Smith, Global regularity of the Navier-Stokes equation on thin three dimensional domains with periodic boundary conditions, Electronic J. Diff. Eqns. 11 (1999), 1-19.

[26] C.L.M.H. Navier, Sur les lois de l'équilibre et du mouvement des corps élastiques. Mem. Acad. R. Sci. Inst. France 6369 (1827).

[27] L. Nirenberg, On elliptic partial differential equations, Ann. Scuola Norm. Sup. Pisa (3) 13 (1959), 115-162. 
[28] M. Paicu and G. Raugel Equations de Navier-Stokes, Graduate course, Université Paris-Sud, manuscript (2006).

[29] C. Parés. Existence, uniqueness and regularity of solution of the equations of a turbulence model for incompressible fluids. Appl. Anal. 43 (1992), no. 3-4, 245-296.

[30] V. Pliss and G. R. Sell, Planetary motion and the climate of the Earth, preprint.

[31] A.G. Ramm Limit of the spectra of the interior Neumann problems when a solid shrinks to a plane one, J. Math. Anal. Appl. 108, (1985), 107-112.

[32] G. Raugel, Dynamics of Partial Differential Equations on Thin Domains, CIME Course, Montecatini Terme, Lecture Notes in Mathematics 1609, Springer Verlag, (1995), 208-315.

[33] G. Raugel and G. R. Sell, Navier-Stokes equations on thin 3D domains. I. Global attractors and global regularity of solutions, J. Amer. Math. Soc. 6 (1993), 503-568.

[34] _ Navier-Stokes equations on thin 3D domains. II. Global regularity of spatially periodic solutions, Nonlinear partial differential equations and their applications. Collège de France Seminar, Vol. XI, Longman Sci. Tech., Harlow, 1994, pp. 205-247.

[35] _ Navier-Stokes equations on thin 3D domains III: Global and local attractors, Turbulence in Fluid Flows: A Dynamical Systems Approach, IMA Volumes in Mathematics and its Applications, Springer, 55 (1993), 137-163.

[36] J. Roh, g-Navier-Stokes equations, University of Minnesota PhD thesis (2001).

[37] M. Schatzman, On the eigenvalues of the Laplace operator on a thin set with Neumann boundary conditions. Appl. Anal. 61 (1996), no. 3-4, 293-306.

[38] G.R. Sell, Global attractors for the three-dimensional Navier-Stokes equations, J Dynamics Differential Equations 8 (1996), 1-33.

[39] G. R. Sell and Y. You, Dynamics of Evolutionary Equations. Applied MAthematical Sciences, Vol 143, Springer (2002).

[40] J. Serrin. Mathematical principles of classical fluid mechanics. Handbuch der Physics vol 8/1 (Berlin: Springer) pp 125-263, (1959).

[41] V. A. Solonnikov and V. E. Šcadilov, On a boundary value problem for a stationary system of NavierStokes equations, Trudy mat. Inst. Steklov 125 (1973), 186-199.

[42] R. Temam, Navier-Stokes equations, third ed., North-Holland, Amsterdam, 1984.

[43] _ Infinite dimensional dynamical systems in mechanics and physics, Springer-Verlag, New York (1988), Second edition (1997).

[44] R. Temam and M. Ziane, Navier-Stokes equations in three-dimensional thin domains with various boundary conditions, Adv. Differential Equations 1 (1996), 499-546.

[45] _ Navier-Stokes equations in thin spherical domains, Contemporary Math. 209 (1997), 281314.

[46] R. Verfürth, Finite element approximation of incompressible Navier-Stokes equations with slip boundary conditions, Numer. Math. 50 (1987), 697-721.

[47] E. Yanagida, Existence of stable stationary solutions of scalar reaction diffusion equations in thin tubular domains, Applicable Anal. 36, (1990),171-188.

D. Iftimie, Université de Lyon, Université Lyon 1, CNRS, UMR 5208 Institut Camille Jordan, BÂtiment DU DoYen JeAn BRACONNIER, 43, BLVD DU 11 NOVEMbRe 1918, F-69622 Villeurbanne Cedex, France. Email: Dragos.iftimie@univ-lyon1.fr

G. Raugel, CNRS, Laboratoire de Mathématiques d'Orsay, Orsay Cedex, F-91405; Univ Paris-Sud, Orsay Cedex, F-91405; Email: Genevieve.Raugel@math.u-Psud.Fr

G. R. Sell, School of Mathematics, University of Minnesota, 206 Church St SE, MinNEAPOLIS Minnesota 55455, USA; EMAIL: SELL@MATH.UMN.EDU 